\title{
Topics in the anabelian geometry of mixed-characteristic local fields
}

\author{
Yuichiro HosHI \\ (Received October 25, 2017) \\ (Revised April 5, 2019)
}

\begin{abstract}
In the present paper, we study the anabelian geometry of mixedcharacteristic local fields by an algorithmic approach. We begin by discussing some generalities on log-shells of mixed-characteristic local fields. One main topic of this discussion is the difference between the log-shell and the ring of integers. This discussion concerning log-shells allows one to establish mono-anabelian reconstruction algorithms for constructing some objects related to the $p$-adic valuations. Next, we consider open homomorphisms between profinite groups of MLF-type. This consideration leads us to a bi-anabelian result for absolutely unramified mixed-characteristic local fields. Next, we establish some mono-anabelian reconstruction algorithms related to each of absolutely abelian mixed-characteristic local fields, mixed-characteristic local fields of degree one, and Galois-specifiable mixed-characteristic local fields. For instance, we give a mono-anabelian reconstruction algorithm for constructing the Norm map with respect to the finite extension determined by the uniquely determined minimal mixed-characteristic local subfield. Finally, we apply various results of the present paper to prove some facts concerning outer automorphisms of the absolute Galois groups of mixedcharacteristic local fields that arise from field automorphisms of the mixed-characteristic local fields.
\end{abstract}

\section{Introduction}

In the present paper, we study the anabelian geometry of mixedcharacteristic local fields. More specifically, we continue our study [cf. [8], [2], [3]] of the mono-anabelian geometry [cf., e.g., [8], Introduction; [8], Remark 1.9.8; [3], Introduction] of mixed-characteristic local fields.

One central object of the study in the present paper is a mixedcharacteristic local field, i.e., an $M L F$. We shall refer to a [field isomorphic to a] finite extension of $\mathbb{Q}_{p}$, for some prime number $p$, as an $M L F$ [cf. [3], Definition 1.1]. If $k$ is an $M L F$, then we shall write

This research was supported by JSPS KAKENHI Grant Number 15K04780.

2010 Mathematics Subject Classification. 11S20.

Key words and phrases. anabelian geometry, mono-anabelian geometry, mono-anabelian reconstruction algorithm, MLF, group of MLF-type, log-shell, absolutely abelian MLF, Galoisspecifiable MLF. 
- $\mathcal{O}_{k} \subseteq k$ for the ring of integers of $k$,

- $\mathfrak{m}_{k} \subseteq \mathcal{O}_{k}$ for the maximal ideal of $\mathcal{O}_{k}$,

- $\underline{k} \stackrel{\text { def }}{=} \mathcal{O}_{k} / \mathrm{m}_{k}$ for the residue field of $\mathcal{O}_{k}$,

- $p_{k} \stackrel{\text { def }}{=} \operatorname{char}(\underline{k})$ for the residue characteristic of $k$,

- $d_{k} \stackrel{\text { def }}{=} \operatorname{dim}_{\mathbb{Q}_{p_{k}}}\left(k_{+}\right), f_{k} \stackrel{\text { def }}{=} \operatorname{dim}_{\mathbb{F}_{p_{k}}}\left(\underline{k}_{+}\right)$[cf. the discussion entitled "Rings" in $\S 0]$,

- $e_{k} \stackrel{\text { def }}{=} \#\left(k^{\times} /\left(\mathcal{O}_{k}^{\times} \cdot p_{k}^{\mathbb{Z}}\right)\right)$ for the absolute ramification index of $k$,

- $k^{(d=1)} \subseteq k$ for the [uniquely determined] minimal MLF contained in $k$,

- $\varepsilon_{k} \stackrel{\text { def }}{=} 1$ (respectively, $\left.\stackrel{\text { def }}{=} 2\right)$ if $p_{k} \neq 2$ (respectively, $p_{k}=2$ ),

- $a_{k}$ for the largest nonnegative integer such that $k$ contains a $p_{k}^{a_{k}}$-th root of unity, and

- $\operatorname{ord}_{k}: k \backslash\{0\} \rightarrow \mathbb{Z}$ for the [uniquely determined] $p_{k}$-adic valuation normalized so that $\operatorname{ord}_{k}$ is surjective [cf. the notational conventions introduced at the beginning of $\S 1$ ]. Moreover, for a positive integer $n$, we use the notation " $\zeta_{n}$ " to denote a primitive $n$-th root of unity.

Another central object of the study in the present paper is a [profinitecf. [3], Proposition 3.3, (i)] group of MLF-type. We shall say that a group is of MLF-type if the group is isomorphic, as an abstract group, to the absolute Galois group of an MLF [cf. [3], Definition 3.1]. If $G$ is a group of MLF-type, then, by applying various mono-anabelian reconstruction algorithms [cf., e.g., [8], Introduction; [8], Remark 1.9.8] of [3], §3, to $G$, we obtain

- a prime number $p(G)$,

- positive integers $d(G), f(G)$, and $e(G)$,

- topological modules $k^{\times}(G)$ and $k_{+}(G)$, and

- a monoid $k_{\times}(G)$

which "correspond" to

- the prime number $p_{k}$,

- the positive integers $d_{k}, f_{k}$, and $e_{k}$,

- the topological modules $k^{\times}$and $k_{+}$[cf. the discussion entitled "Rings" in $\S 0]$, and

- the monoid $k_{\times}$[cf. the discussion entitled "Fields" in $\S 0$ ],

respectively [cf. [3], Summary 3.15]. Moreover, by applying the monoanabelian reconstruction algorithms of Definition 2.4, (i), (ii), of the present paper to $G$, we obtain

- nonnegative integers $\varepsilon(G)$ and $a(G)$

which "correspond" to

- the nonnegative integers $\varepsilon_{k}$ and $a_{k}$,

respectively [cf. Proposition 2.5, (i), of the present paper]. 
In $\S 1$, we discuss some generalities on log-shells of MLF's. If $k$ is an MLF, then we shall refer to the compact open topological submodule

$$
\mathscr{I}_{k} \stackrel{\text { def }}{=} \frac{1}{2 p_{k}} \cdot \log _{k}\left(\mathcal{O}_{k}^{\times}\right) \subseteq k_{+}
$$

-where we write $\log _{k}: \mathcal{O}_{k}^{\times} \rightarrow k_{+}$for the $p_{k}$-adic logarithm-of the topological module $k_{+}$as the log-shell of $k$ [cf. [8], Definition 5.4, (iii)]. As is well-known [cf., e.g., [3], Lemma 1.2, (vi)], the log-shell contains the compact open topological submodule $\left(\mathcal{O}_{k}\right)_{+} \subseteq k_{+}$of $k_{+}$:

$$
\left(\mathcal{O}_{k}\right)_{+} \subseteq \mathscr{I}_{k}
$$

One main topic of the study of $\S 1$ is the difference between $\left(\mathcal{O}_{k}\right)_{+}$and $\mathscr{I}_{k}$. In $\S 1$, we prove, for instance, the following result [cf. Proposition 1.5; Lemma 1.8, (i); Proposition 1.10, (i)].

THEOREM A. Let $k$ be an MLF. Then the following hold:

(i) The quotient

$$
\mathscr{I}_{k} /\left(\mathcal{O}_{k}\right)_{+}
$$

is isomorphic, as an abstract module, to the module defined by

$$
\prod_{v=1}^{\infty}\left(\mathbb{Z}_{+} / p_{k}^{v} \mathbb{Z}_{+}\right)^{\oplus b_{k}(v)-\delta\left(v, a_{k}\right)}
$$

-where we write

$$
b_{k}(v) \stackrel{\text { def }}{=}\left(\left\lfloor\frac{\varepsilon_{k} \cdot e_{k}-1}{p_{k}^{v-1}}\right\rfloor-2 \cdot\left\lfloor\frac{\varepsilon_{k} \cdot e_{k}-1}{p_{k}^{v}}\right\rfloor+\left\lfloor\frac{\varepsilon_{k} \cdot e_{k}-1}{p_{k}^{v+1}}\right\rfloor\right) \cdot f_{k}
$$

and $\delta(i, j) \stackrel{\text { def }}{=} 1$ (respectively, $\stackrel{\text { def }}{=} 0$ ) if $i=j$ (respectively, $i \neq j$ ). In particular, the isomorphism class of $\mathscr{I}_{k} /\left(\mathcal{O}_{k}\right)_{+}$depends only on $p_{k}, f_{k}, e_{k}$, and $a_{k}$.

(ii) It holds that the submodule $\mathscr{I}_{k} \subseteq k_{+}$coincides with the submodule $\left(\mathcal{O}_{k}\right)_{+} \subseteq k_{+}$if and only if one of the following three conditions is satisfied:

- The prime number $p_{k}$ is odd, and, moreover, the finite extension $k / k^{(d=1)}$ is unramified.

- The field $k$ is isomorphic to the field $\mathbb{Q}_{2}$.

- The field $k$ is isomorphic to the field $\mathbb{Q}_{3}\left(\zeta_{3}\right)$.

(iii) We shall define a nonnegative integer

as follows: 
- If either $p_{k} \geq 5$ or $k$ is not isomorphic to $\mathbb{Q}_{p_{k}}\left(\zeta_{p_{k}} a_{k}\right)$, then

$$
v_{k} \stackrel{\text { def }}{=} \min \left\{v \geq 0 \mid \varepsilon_{k} \cdot e_{k} \leq p_{k}^{v}\right\} .
$$

- If $p_{k} \leq 3$, and $k$ is isomorphic to $\mathbb{Q}_{p_{k}}\left(\zeta_{p_{k}} a_{k}\right)$, then $v_{k} \stackrel{\text { def }}{=} \min \left\{v \geq 0 \mid \varepsilon_{k} \cdot e_{k} \leq p_{k}^{v+1}\right\}=\min \left\{v \geq 1 \mid \varepsilon_{k} \cdot e_{k} \leq p_{k}^{v}\right\}-1$.

Then the nonnegative integer $v_{k}$ is the smallest integer such that

$$
p_{k}^{v_{k}} \cdot \mathscr{I}_{k} \subseteq\left(\mathcal{O}_{k}\right)_{+} \subseteq \mathscr{I}_{k} .
$$

The various results of $\S 1$ may be regarded as "preparatory portions" for the establishment of mono-anabelian reconstruction algorithms of $\S 2$.

In $\S 2$, we establish mono-anabelian reconstruction algorithms for constructing, from a group $G$ of MLF-type,

- a homomorphism of modules

$$
\operatorname{ord}_{\llbracket}(G): k^{\times}(G) \rightarrow \mathbb{Z}_{+}
$$

[cf. Definition 2.2] which "corresponds" [cf. Proposition 2.3] to the $p_{k}$-adic valuation $\operatorname{ord}_{k}: k \backslash\{0\} \rightarrow \mathbb{Z}$ and

- a map of sets

$$
\operatorname{ord}_{\boxplus}(G): k_{+}(G) \backslash\{0\} \rightarrow \mathbb{Z}
$$

[cf. Definition 2.6, (ii)] which "corresponds" [cf. Proposition 2.7, (ii)] to a

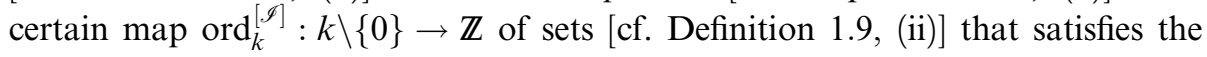
following condition [cf. Proposition 1.10, (ii)]: For each $a \in k \backslash\{0\}$, it holds that

$$
\operatorname{ord}_{k}(a) \leq \operatorname{ord}_{k}^{[\mathscr{l}]}(a)<\operatorname{ord}_{k}(a)+e_{k} \cdot\left(v_{k}+1\right)
$$

[cf. Theorem A, (iii)], i.e., a sort of " $p_{k}$-adic valuation with an indeterminacy" [cf. Remark 1.10.1; also Remark 2.11.1].

Moreover, we also establish mono-anabelian reconstruction algorithms for constructing, from a group $G$ of MLF-type such that $\varepsilon(G) \cdot e(G)=f(G)+a(G)$ [cf. also Remark 2.11.2], topological submodules

$$
\mathrm{m}^{n}(G) \subseteq \mathcal{O}_{+}(G) \subseteq k_{+}(G)
$$

[cf. Definition 2.9, (i), (ii)] - where $n$ is a nonnegative integer-of $k_{+}(G)$ which "correspond" [cf. Proposition 2.10] to the topological submodules $\mathfrak{m}_{k}^{n} \subseteq\left(\mathcal{O}_{k}\right)_{+} \subseteq k_{+}$of $k_{+}$, respectively.

In $\S 3$, we consider open homomorphisms between profinite groups of MLF-type. One main application of the results of $\S 3$ is as follows [cf. Theorem 3.6, Corollary 3.7]. 
Theorem B. For each $\square \in\{\circ, \bullet\}$, let $G_{\square}$ be a profinite group of MLFtype. Let

$$
\alpha: G_{\circ} \rightarrow G_{\bullet}
$$

be an open homomorphism. Then the following hold:

(i) Suppose that $d\left(G_{\circ}\right) \leq d\left(G_{\bullet}\right)$ [which is the case if, for instance, $\left.d\left(G_{\circ}\right)=1\right]$. Then $\alpha$ is an isomorphism.

(ii) Suppose that $e\left(G_{\circ}\right) \leq e\left(G_{\bullet}\right)$ [which is the case if, for instance, $\left.e\left(G_{\circ}\right)=1\right]$. Then $\alpha$ is injective.

Theorem B leads us to the following bi-anabelian [cf., e.g., [8], Introduction; [8], Remark 1.9.8; [3], Introduction] result [cf. Corollary 3.8].

THeOREM C. For each $\square \in\{0, \bullet\}$, let $k_{\square}$ be an MLF and $\bar{k}_{\square}$ an algebraic closure of $k_{\square}$; write $G_{\square} \stackrel{\text { def }}{=} \operatorname{Gal}\left(\bar{k}_{\square} / k_{\square}\right)$. Suppose that $e_{k_{\circ}}=1$. Then it holds that the field $k_{\circ}$ is isomorphic to the field $k_{\bullet}$ if and only if there exists a surjection $G_{\circ} \rightarrow G_{\bullet}$.

In $\S 4$, we discuss some mono-anabelian reconstruction algorithms related to absolutely abelian MLF's. We shall say that an MLF $k$ is absolutely abelian if the finite extension $k / k^{(d=1)}$ is Galois, and the Galois group is abelian [cf. Definition 4.2, (ii)]. In $\S 4$, we establish, for instance, a mono-anabelian reconstruction algorithm for constructing, from a group $G$ of MLF-type, a homomorphism of topological modules

$$
\mathrm{Nm}_{\mathrm{abs}}(G)
$$

[cf. Definition 4.7, (iii)] which "corresponds" [cf. Proposition 4.9, (i)] to the Norm map $\mathrm{Nm}_{k / k^{(d=1)}}: k^{\times} \rightarrow\left(k^{(d=1)}\right)^{\times}$with respect to the finite extension $k / k^{(d=1)}$. This homomorphism $\operatorname{Nm}_{\text {abs }}(G)$ allows one to define the notion of $M L F-$ Galois label of $G$, i.e., the triple consisting of the prime number $p(G)$, the positive integer $d(G)$, and the image of the homomorphism $\mathrm{Nm}_{\mathrm{abs}}(G)$ [ $\mathrm{cf}$. Definition 4.10]. By applying the main theorems of [4] and [13], we obtain the following result [cf. Theorem 4.11].

Theorem D. For each $\square \in\{0, \bullet\}$, let $G_{\square}$ be a group of MLF-type. Suppose that $\left\{\left(p\left(G_{\circ}\right), a\left(G_{\circ}\right)\right),\left(p\left(G_{\bullet}\right), a\left(G_{\bullet}\right)\right)\right\} \nsubseteq\{(2,1)\}$. Then it holds that the group $G_{\circ}$ is isomorphic to the group $G_{\bullet}$ if and only if the MLF-Galois label of $G_{\circ}$ coincides with the MLF-Galois label of $G_{\text {. }}$.

Moreover, in $\S 4$, we also obtain the following bi-anabelian result [cf. Corollary 4.14].

THEOREM E. For each $\square \in\{0, \bullet\}$, let $k_{\square}$ be an $M L F$ and $\bar{k}_{\square}$ an algebraic closure of $k_{\square}$; write $G_{\square} \stackrel{\text { def }}{=} \operatorname{Gal}\left(\bar{k}_{\square} / k_{\square}\right)$. Suppose that there exists a surjection 
$G_{\circ} \rightarrow G_{\bullet}$ [which thus implies that $p_{k_{\circ}}=p_{k_{\bullet}}-c f$. Proposition 3.4, (iii)] compatible with the respective $p_{k_{0}}$-adic, i.e., $p_{k_{0}}$-adic, cyclotomic characters [which is the case if, for instance, the surjection $G_{\circ} \rightarrow G_{\bullet}$ is an isomorphism-cf. [3], Proposition 4.2, (iv)]. Then the following hold:

(i) The [uniquely determined] maximal absolutely abelian MLF contained in $k_{\circ}$ is isomorphic to the [uniquely determined] maximal absolutely abelian MLF contained in $k_{\bullet}$.

(ii) Suppose that $k_{\circ}$ is absolutely abelian. Then the field $k_{\circ}$ is isomorphic to the field $k_{\text {. }}$.

Here, observe that Theorem E, (i), may be regarded as a refinement of the main theorem of [6] [cf. Remark 4.14.1].

In $\S 5$, we discuss some mono-anabelian reconstruction algorithms related to MLF's of degree one, i.e., such that the integer " $d_{(-)}$" is equal to one. For instance, we establish a mono-anabelian reconstruction algorithm for constructing, from a group $G$ of MLF-type such that $d(G)=1$ [cf. Remark 5.10.1], a structure of topological field on $k_{\times}(G)$ [cf. Definition 5.2] which "corresponds" [cf. Theorem 5.4, (i)] to the topological field structure of $k$, i.e., on $k_{\times}$.

In $\S 6$, we discuss Galois-specifiable MLF's. We shall say that an MLF $k$ is Galois-specifiable if $k$ is Galois over $k^{(d=1)}$, and, moreover, the following condition is satisfied: If $L$ is an MLF such that the absolute Galois group of $k$ is isomorphic to the absolute Galois group of $L$, then the field $k$ is isomorphic to the field $L$ [cf. Definition 6.1]. We prove the following result [cf. Theorem 5.9, (ii); Remark 5.9.1; Theorem 6.3; Remark 6.3.1].

THEOREM F. Let $k$ be an MLF. Consider the following five conditions:

(1) The $M L F k$ is absolutely abelian [cf. Definition 4.2, (ii)].

(2) The MLF $k$ is Galois-specifiable [cf. Definition 6.1].

(3) The $M L F k$ is absolutely strictly radical [cf. Definition 5.6, (iii)].

(4) The MLF $k$ is absolutely characteristic [cf. Definition 5.7].

(5) The MLF $k$ is absolutely Galois [cf. Definition 4.2, (i)].

Then the following hold:

(i) The implications

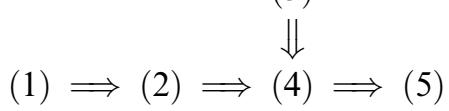

hold.

(ii) Suppose that $\left(p_{k}, a_{k}\right) \neq(2,1)$. Then the equivalence

$$
(1) \Leftrightarrow(2)
$$

holds. 
(iii) There exists an MLF that violates the implication (4) $\Rightarrow(2)$ (respectively, (4) $\Rightarrow(3) ;(5) \Rightarrow(4))$.

Moreover, in the present paper, we observe that the condition for an MLF to be absolutely abelian and the condition for an MLF to be Galois-specifiable may be considered to be "group-theoretic" [cf. Remark 4.15.1, (i); Remark 6.13.1], but each of the condition for an MLF to be absolutely strictly radical, the condition for an MLF to be absolutely characteristic, and the condition for an MLF to be absolutely Galois should be considered to be "not grouptheoretic" [cf. Remark 4.15.1, (ii); Remark 5.9.2].

Let $k$ be an MLF and $\bar{k}$ an algebraic closure of $k$. Write $G_{k} \stackrel{\text { def }}{=} \operatorname{Gal}(\bar{k} / k)$. Then let us recall that we have a natural injection $\operatorname{Aut}(k) \hookrightarrow \operatorname{Out}\left(G_{k}\right)$ [cf., e.g., [3], Proposition 2.1]. By means of this injection, let us regard $\operatorname{Aut}(k)$ as a subgroup of $\operatorname{Out}\left(G_{k}\right)$ :

$$
\operatorname{Aut}(k) \subseteq \operatorname{Out}\left(G_{k}\right)
$$

In $\S 6$, we also establish a mono-anabelian reconstruction algorithm for constructing, from a group $G$ of MLF-type that satisfies a certain condition [cf. Definition 6.8, (i)] "corresponding" [cf. Theorem 6.10] to the condition for an MLF to be Galois-specifiable, a collection

$$
\operatorname{Orb}_{\mathrm{sqg}}(G)
$$

[cf. Definition 6.8, (ii)] of subgroups of $\operatorname{Out}(G)$ which "corresponds" [cf. Theorem 6.12, (ii)] to the $\operatorname{Out}\left(G_{k}\right)$-orbit, i.e., by conjugation, of the subgroup $\operatorname{Aut}(k) \subseteq \operatorname{Out}\left(G_{k}\right)$.

In $\S 7$ and $\S 8$, we discuss outer automorphisms of the absolute Galois groups of MLF's that arise from field automorphisms of the MLF's. For instance, we prove the following result [cf. Theorem 7.2, (i); Theorem 7.5; Corollary 8.7].

THEOREM G. Let $k$ be an MLF and $\bar{k}$ an algebraic closure of $k$. Write $G_{k} \stackrel{\text { def }}{=} \operatorname{Gal}(\bar{k} / k)$. Then the following hold:

(i) Suppose that the MLF $k$ is absolutely characteristic, and that $p_{k}$ is odd. Then the subgroup

$$
\operatorname{Aut}(k) \subseteq \operatorname{Out}\left(G_{k}\right)
$$

is not normally terminal [cf. the discussion entitled "Groups" in §0].

(ii) Write $k^{(\mathrm{ab})} \subseteq k$ for the [uniquely determined] maximal absolutely abelian MLF contained in $k$. Suppose that a maximal intermediate field of $k / k^{(\mathrm{ab})}$ tamely ramified over $k^{(\mathrm{ab})}$ does not coincide with $k^{(d=1)}$ [which is the case if, for instance, $\left.k^{(\mathrm{ab})} \neq k^{(d=1)}\right]$, and that $\left(p_{k}, a_{k}\right) \neq(2,1)$. Let $n$ be a non- 
negative integer such that $\left[k: k^{(\mathrm{ab})}\right] \in p_{k}^{n} \mathbb{Z}$ and $A$ an abelian $p_{k}$-group that satisfies the following two conditions:

(1) It holds that $\# A=p_{k}^{n}$.

(2) The finite abelian group $A$ is generated by at most $\left(d_{k} / p_{k}^{n}\right)-1$ elements.

Then there exists a subgroup of $\operatorname{Out}\left(G_{k}\right)$ isomorphic to $A$.

(iii) Suppose that $p_{k}$ is odd, and that

$$
k=\mathbb{Q}_{p_{k}}\left(\zeta_{p_{k}}, p_{k}^{1 / p_{k}}\right) \text {. }
$$

Then the subgroup

$$
\operatorname{Aut}(k) \subseteq \operatorname{Out}\left(G_{k}\right)
$$

is neither normally terminal nor normal.

One motivation of studying Theorem $\mathrm{G}$ is as follows [cf. Remark 7.5.2]: Let $k$ be an MLF and $\bar{k}$ an algebraic closure of $k$. Write $G_{k} \stackrel{\text { def }}{=} \operatorname{Gal}(\bar{k} / k)$. Then, as is well-known [cf., e.g., the discussion given at the final portion of [12], Chapter VII, §5], in general, the natural injection

$$
\operatorname{Aut}(k) \hookrightarrow \operatorname{Out}\left(G_{k}\right)
$$

is not surjective. Under this state of affairs, one may consider the following problem:

Problem: Is there a certain "suitable" characterization of the sub$\operatorname{group} \operatorname{Aut}(k) \subseteq \operatorname{Out}\left(G_{k}\right)$ of $\operatorname{Out}\left(G_{k}\right)$ ?

[Here, let us observe that

the mono-anabelian reconstruction algorithm of " $\mathrm{Orb}_{\mathrm{sqg}}(G)$ " in the discussion preceding Theorem $G$ may be regarded as a certain affirmative solution to this problem, i.e., in the case where the MLF $k$ is Galois-specifiable.]

From the point of view of this problem, let us observe the [easily verified] finiteness of the $\operatorname{group} \operatorname{Aut}(k)$.

In particular, as one of possible solutions to the above problem, one may discuss the following question:

$\left(*_{\text {fin }}\right) \quad$ Is the subgroup $\operatorname{Aut}(k)$ of $\operatorname{Out}\left(G_{k}\right)$ the uniquely determined maximal finite subgroup of $\operatorname{Out}\left(G_{k}\right)$ ? Put another way, is every element of $\operatorname{Out}\left(G_{k}\right)$ of finite order contained in the subgroup $\operatorname{Aut}(k)$ of $\operatorname{Out}\left(G_{k}\right)$ ?

Now let us observe that it is immediate that an affirmative answer to this question $\left(*_{\text {fin }}\right)$ implies an affirmative answer to the following question $\left(*_{\text {char }}\right)$, hence also an affirmative answer to the following question $\left(*_{\text {nor }}\right)$ : 
$\left(*_{\text {char }}\right) \quad$ Is the subgroup $\operatorname{Aut}(k)$ of $\operatorname{Out}\left(G_{k}\right)$ characteristic?

$\left(*_{\text {nor }}\right) \quad$ Is the subgroup $\operatorname{Aut}(k)$ of $\operatorname{Out}\left(G_{k}\right)$ normal?

Then one may easily find that

- Theorem G, (i), is related to the question $\left(*_{\text {nor }}\right)$,

- Theorem G, (ii) [cf. also the example in Remark 7.5.1], yields a negative answer to the question $\left(*_{\mathrm{fin}}\right)$, and

- Theorem G, (iii), yields a negative answer to the question $\left(*_{\text {nor }}\right)$, hence also negative answers to the questions $\left(*_{\text {fin }}\right)$ and $\left(*_{\text {char }}\right)$.

This is one motivation of studying Theorem G.

Finally, in Remark 8.7.1, we recall some of the discussions of $\S 8$ from the point of view of the notion of "link" [cf. [9], §2.7, (i)].

\section{Notations and conventions}

Numbers. If $a \in \mathbb{Q}$ is a rational number, then we shall write $\lfloor a\rfloor \in \mathbb{Z}$ for the largest integer such that $\lfloor a\rfloor \leq a$.

SETS. If $S$ is a finite set, then we shall write $\# S$ for the cardinality of $S$. If $G$ is a group, and $T$ is a set equipped with an action of $G$, then we shall write $T^{G} \subseteq T$ for the subset of $G$-invariants of $T$.

MonoIDs. In the present paper, every "monoid" is assumed to be commutative. Let $M$ be a [multiplicative] monoid. We shall write $M^{\times} \subseteq M$ for the abelian group of invertible elements of $M$. We shall write $M^{\text {gp }}$ for the groupification of $M$ [i.e., the abelian group given by the set of equivalence classes with respect to the relation $\sim$ on $M \times M$ defined by, for $\left(a_{1}, b_{1}\right)$, $\left(a_{2}, b_{2}\right) \in M \times M,\left(a_{1}, b_{1}\right) \sim\left(a_{2}, b_{2}\right)$ if there exists an element $c \in M$ of $M$ such that $c a_{1} b_{2}=c a_{2} b_{1}$ ]. We shall write $M^{\mathrm{pf}}$ for the perfection of $M$ [i.e., the monoid obtained by forming the inductive limit of the inductive system of monoids

$$
\cdots \rightarrow M \rightarrow M \rightarrow \cdots
$$

given by assigning to each positive integer $n$ a copy of $M$, which we denote by $I_{n}$, and to each two positive integers $n, m$ such that $n$ divides $m$ the homomorphism $I_{n}=M \rightarrow I_{m}=M$ given by multiplication by $m / n$ ]. We shall write $M^{\circledast} \stackrel{\text { def }}{=} M \cup\left\{*_{M}\right\}$; we regard $M^{\circledast}$ as a monoid [that contains $M$ as a submonoid] by setting $*_{M} \cdot *_{M} \stackrel{\text { def }}{=} *_{M}$ and $a \cdot *_{M} \stackrel{\text { def }}{=} *_{M}$ for every $a \in M$.

Modules. Let $M$ be a module. If $n$ is a positive integer, then we shall write $M[n] \subseteq M$ for the submodule obtained by forming the kernel of the 
endomorphism of $M$ given by multiplication by $n$. We shall write $M_{\text {tor }} \stackrel{\text { def }}{=}$ $\bigcup_{n \geq 1} M[n] \subseteq M$ for the submodule of torsion elements of $M$ and

$$
M^{\wedge} \stackrel{\text { def }}{=} \underset{\leftarrow}{\lim } M /(n \cdot M)
$$

- where the projective limit is taken over the positive integers $n$. [So if $M$ is finitely generated, then $M^{\wedge}$ coincides with the profinite completion of $M$.]

Groups. Let $G$ be a group and $H \subseteq G$ a subgroup of $G$. We shall write $Z_{G}(H) \subseteq G$ for the centralizer of $H$ in $G$ [i.e., the subgroup consisting of $g \in G$ such that $g h=h g$ for every $h \in H]$ and $N_{G}(H) \subseteq G$ for the normalizer of $H$ in $G$ [i.e., the subgroup consisting of $g \in G$ such that $g H=H g$ ]. We shall say that $H$ is normally terminal in $G$ if $N_{G}(H)=H$, or, alternatively, $N_{G}(H) \subseteq H$.

TOPOLOGICAL GROUPS. If $G$ is a topological group, then we shall write $G^{\mathrm{ab}}$ for the abelianization of $G$ [i.e., the quotient of $G$ by the closure of

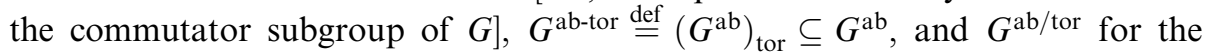
quotient of $G^{\mathrm{ab}}$ by the closure of $G^{\mathrm{ab} \text {-tor }} \subseteq G^{\mathrm{ab}}$. If $H$ is a profinite group, and $p$ is a prime number, then we shall write $H^{(p)}$ for the maximal pro-p quotient of $H$.

Rings. In the present paper, every "ring" is assumed to be unital, associative, and commutative. Let $R$ be a ring. We shall write $R_{+}$for the underlying additive module of $R$ and $R^{\times} \subseteq R$ for the multiplicative group of units of $R$. If, moreover, $R$ is an integral domain, then we shall write $R^{\triangleright} \subseteq R$ for the multiplicative monoid of nonzero elements of $R$. [So if $R$ is an integral domain, then we have a natural inclusion $R^{\times} \subseteq R^{\triangleright}$ of monoids.]

FIELDS. Let $K$ be a field [i.e., an integral domain such that $K^{\times}=K^{\triangleright}$ ]. We shall write $\boldsymbol{\mu}(K) \stackrel{\text { def }}{=}\left(K^{\times}\right)_{\text {tor }}$ for the group of roots of unity in $K$ and $K_{\times}=K^{\times} \cup\{0\}$ for the underlying multiplicative monoid of $K$. [So we have a natural isomorphism $\left(K^{\times}\right)^{\circledast} \stackrel{\sim}{\rightarrow} K_{\times}$of monoids that maps $*_{K^{\times}}$to 0 .] If, moreover, $K$ is algebraically closed and of characteristic zero, then we shall write

$$
\Lambda(K) \stackrel{\text { def }}{=} \underset{⿱}{\lim } \boldsymbol{\mu}(K)[n]=\underset{\leftarrow}{\lim } K^{\times}[n]
$$

- where the projective limits are taken over the positive integers $n$ - and refer to $\Lambda(K)$ as the cyclotome associated to $K$. Thus, the cyclotome has a natural structure of profinite, hence also topological, module and is isomorphic, as an abstract topological module, to $\hat{\mathbb{Z}}_{+}$. 


\section{Generalities on log-shells}

In the present $\S 1$, let

$$
k
$$

be an $M L F$-i.e., a [field isomorphic to a] finite extension of $\mathbb{Q}_{p}$, for some prime number $p$ [cf. [3], Definition 1.1] —and

\section{$\bar{k}$}

an algebraic closure of $k$. We shall write

- $\mathcal{O}_{k} \subseteq k$ for the ring of integers of $k$,

- $\mathrm{m}_{k} \subseteq \mathcal{O}_{k}$ for the maximal ideal of $\mathcal{O}_{k}$,

- $\underline{k} \stackrel{\text { def }}{=} \mathcal{O}_{k} / \mathrm{m}_{k}$ for the residue field of $\mathcal{O}_{k}$,

- $\mathcal{O}_{k}^{\prec n} \stackrel{\text { def }}{=} 1+\mathfrak{m}_{k}^{n} \subseteq \mathcal{O}_{k}^{\times}$[where $n$ is a positive integer] for the $n$-th higher unit group of $\mathcal{O}_{k}$,

- $\mathcal{O}_{k}^{<} \stackrel{\text { def }}{=} \mathcal{O}_{k}^{<1}$ for the group of principal units of $\mathcal{O}_{k}$,

- $\mu_{k}$ for the [uniquely determined] Haar measure on [the locally compact topological module] $k_{+}$normalized so that $\mu_{k}\left(\left(\mathcal{O}_{k}\right)_{+}\right)=1$,

- $p_{k} \stackrel{\text { def }}{=} \operatorname{char}(\underline{k})$ for the residue characteristic of $k$,

- $d_{k} \stackrel{\text { def }}{=} \operatorname{dim}_{\mathbb{Q}_{p_{k}}}\left(k_{+}\right)$,

- $f_{k} \stackrel{\text { def }}{=} \operatorname{dim}_{\mathbb{F}_{p_{k}}}\left(\underline{k}_{+}\right)$,

- $e_{k} \stackrel{\text { def }}{=} \#\left(k^{\times} /\left(\mathcal{O}_{k}^{\times} \cdot p_{k}^{\mathbb{Z}}\right)\right)$ for the absolute ramification index of $k$,

- $\log _{k}: \mathcal{O}_{k}^{\times} \rightarrow k_{+}$for the $p_{k}$-adic logarithm,

- $\mathscr{I}_{k} \stackrel{\text { def }}{=}\left(2 p_{k}\right)^{-1} \cdot \log _{k}\left(\mathcal{O}_{k}^{\times}\right) \subseteq k_{+}$for the $\log$-shell of $k$,

- $\mathcal{O}_{\bar{k}} \subseteq \bar{k}$ for the ring of integers of $\bar{k}$,

- $\underline{k}$ for the residue field of $\mathcal{O}_{\bar{k}}$,

- $G_{k} \stackrel{\text { def }}{=} \mathrm{Gal}(\bar{k} / k)$,

- $I_{k} \subseteq G_{k}$ for the inertia subgroup of $G_{k}$,

- $P_{k} \subseteq I_{k}$ for the wild inertia subgroup of $G_{k}$, and

- $\operatorname{Frob}_{k} \in \operatorname{Gal}(\underline{k} / \underline{k}) \simeq G_{k} / I_{k}$ for the [\# $\underline{k}$-th power] Frobenius element

[cf. the notational conventions introduced in the discussions following [3], Definition 1.1, and [3], Lemma 1.3]. We shall write, moreover,

- $k^{(d=1)} \subseteq k$ for the [uniquely determined] minimal MLF contained in $k$,

- $e_{k}^{[\boldsymbol{\mu}]}=\left\lfloor e_{k} /\left(p_{k}-1\right)\right\rfloor$,

- $\varepsilon_{k} \stackrel{\text { def }}{=} 1$ (respectively, $\stackrel{\text { def }}{=} 2$ ) if $p_{k} \neq 2$ (respectively, $p_{k}=2$ ) [cf. [3], Lemma 1.3, (iii)],

- $a_{k}$ for the largest nonnegative integer such that $k$ contains a $p_{k}^{a_{k}}$-th root of unity [i.e. the " $a$ " in [3], Lemma 1.2, (i)],

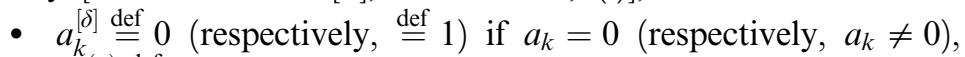

- $\mathscr{I}_{k}^{(n)} \stackrel{\text { def }}{=}\left(2 p_{k}\right)^{-1} \cdot \log _{k}\left(\mathcal{O}_{k}^{<n}\right) \subseteq \mathscr{I}_{k}$ [where $n$ is a positive integer], and 
- $\operatorname{ord}_{k}: k \backslash\{0\} \rightarrow \mathbb{Z}$ for the [uniquely determined] $p_{k}$-adic valuation normalized so that $\operatorname{ord}_{k}$ is surjective.

Finally, for each positive integer $n$, let

$$
\zeta_{n} \in \bar{k}
$$

be a primitive $n$-th root of unity.

In the present $\S 1$, we discuss some generalities on log-shells of MLF's.

Proposition 1.1. The following hold:

(i) It holds that $\mathscr{I}_{k}^{(1)}=\mathscr{I}_{k}$.

(ii) It holds that $\mu_{k}\left(\mathscr{I}_{k}\right)=p_{k}^{\varepsilon_{k}} \cdot d_{k}-f_{k}-a_{k}$.

(iii) Let $n$ be an integer such that $n>e_{k}^{[\mu]}$. Then it holds that $\mathscr{I}_{k}^{(n)}=$ $\mathrm{m}_{k}^{n-\varepsilon_{k} \cdot e_{k}}$.

(iv) If $a_{k}^{[\delta]}=1$, then it holds that $\left(f_{k}, e_{k}\right)=\left(1, p_{k}^{a_{k}-1} \cdot\left(p_{k}-1\right)\right)$ if and only if $k$ is isomorphic to $\mathbb{Q}_{p_{k}}\left(\zeta_{p_{k}} a_{k}\right)$.

(v) It holds that $p_{k}^{a_{k}-1} \cdot\left(p_{k}-1\right) \leq e_{k}$. If, moreover, $a_{k}^{[\delta]}=1$, then it holds that $e_{k} \in p_{k}^{a_{k}-1} \cdot\left(p_{k}-1\right) \cdot \mathbb{Z}$.

Proof. Assertion (i) follows from [3], Lemma 1.2, (i), (ii), (v). Assertion (ii) is the content of [3], Lemma 1.3, (iii). Assertion (iii) follows from [11], Chapter II, Proposition 5.5. Finally, since $\left(f_{\mathbb{Q}_{p_{k}}\left(\zeta_{p_{k}} a_{k}\right)}, e_{\mathbb{Q}_{p_{k}}\left(\zeta_{p_{k}} a_{k}\right)}\right)=\left(1, p_{k}^{a_{k}-1}\right.$. $\left.\left(p_{k}-1\right)\right)$ if $a_{k}^{[\delta]}=1$ [cf. [11], Chapter II, Proposition 7.13, (i)], assertions (iv), (v) follow immediately from the [easily verified] fact that $k$ always contains an MLF isomorphic to $\mathbb{Q}_{p_{k}}\left(\zeta_{p_{k}}^{a_{k}}\right)$. This completes the proof of Proposition 1.1.

Lemma 1.2. Let $a \in k \backslash\{0\}$ be an element of $k \backslash\{0\}$. Then the integer $\operatorname{ord}_{k}(a) \in \mathbb{Z}$ coincides with the uniquely determined integer $n$ such that Frob $_{k}^{n} \in$ $G_{k} / I_{k}$ coincides with the image of $a \in k \backslash\{0\}$ by the composite of the injective homomorphism $\operatorname{rec}_{k}: k^{\times} \hookrightarrow G_{k}^{\mathrm{ab}}$ of [3], Lemma 1.7, and the natural surjection $G_{k}^{\mathrm{ab}} \rightarrow G_{k} / I_{k}[c f$. [3], Lemma 1.5, (i)].

Proof. This assertion follows immediately from [3], Lemma 1.7, (1).

LEMMA 1.3. The following hold:

(i) Suppose that $a_{k}^{[\delta]}=1$. Let $v$ be an integer such that $1 \leq v \leq a_{k}$. Then it holds that $\zeta_{p_{k}^{v}} \in \mathcal{O}_{k}^{\prec \prec e_{k}^{[\mu]} / p_{k}^{v-1}}\left[\right.$ cf. Proposition 1.1, (v)] but $\zeta_{p_{k}^{v}} \notin \mathcal{O}_{k}^{\prec\left(e_{k}^{[\mu]} / p_{k}^{v-1}\right)+1}$.

(ii) Let $n$ be a positive integer. Then the modules $\mathcal{O}_{k}^{<n} / \mathcal{O}_{k}^{<n+1}, \mathscr{I}_{k}^{(n)} / \mathscr{I}_{k}^{(n+1)}$ are annihilated by $p_{k}$. In particular, these modules have respective natural structures of $\mathbb{F}_{p_{k}}$-vector spaces. Moreover, the $\mathbb{F}_{p_{k}}$-vector space $\mathcal{O}_{k}^{\prec n} / \mathcal{O}_{k}^{\prec n+1}$ is of dimension $f_{k}$. 
(iii) Let $n$ be a positive integer. Then the $p_{k}$-adic logarithm $\log _{k}: \mathcal{O}_{k}^{\times} \rightarrow$ $k_{+}$determines a surjection of $\mathbb{F}_{p_{k}}$-vector spaces [cf. (ii)]

$$
\mathcal{O}_{k}^{\prec n} / \mathcal{O}_{k}^{\prec n+1} \rightarrow \mathscr{I}_{k}^{(n)} / \mathscr{I}_{k}^{(n+1)} .
$$

(iv) In the situation of (iii), if the integer $n$ is of the form " $e_{k}^{[\boldsymbol{\mu}]} / p_{k}^{v-1}$ " for some integer $v$ such that $1 \leq v \leq a_{k}$, then the kernel of the surjection of (iii) is generated by the image of $\zeta_{p_{k}^{v}} \in \mathcal{O}_{k}^{\prec e_{k}^{[\mu]} / p_{k}^{v-1}}[c f$. (i)] [hence also of dimension one over $\left.\mathbb{F}_{p_{k}}\right]$. If the integer $n$ is not of the form " $e_{k}^{[\mu]} / p_{k}^{v-1}$ " for any integer $v$ such that $1 \leq v \leq a_{k}$, then the surjection of (iii) is an isomorphism.

Proof. Assertion (i) follows immediately from Proposition 1.1, (iv), together with [11], Chapter II, Proposition 7.13, (iv). Assertions (ii), (iii) follow from [11], Chapter II, Proposition 3.10, together with the definition of " $\mathscr{I}_{k}^{(n)}$ ", Assertion (iv) follows immediately from assertion (i), together with [3], Lemma 1.2, (ii), (v). This completes the proof of Lemma 1.3.

DEFINITION 1.4 .

(i) For each positive integer $v$, we shall write

$$
b_{k}(v) \stackrel{\text { def }}{=}\left(\left\lfloor\frac{\varepsilon_{k} \cdot e_{k}-1}{p_{k}^{v-1}}\right\rfloor-2 \cdot\left\lfloor\frac{\varepsilon_{k} \cdot e_{k}-1}{p_{k}^{v}}\right\rfloor+\left\lfloor\frac{\varepsilon_{k} \cdot e_{k}-1}{p_{k}^{v+1}}\right\rfloor\right) \cdot f_{k} .
$$

Moreover, we shall write

$$
b_{k}(0) \stackrel{\text { def }}{=} \infty \text {. }
$$

(ii) We shall write

$$
\mathbb{I}_{k} \stackrel{\text { def }}{=} \prod_{v=1}^{\infty}\left(\mathbb{Z}_{+} / p_{k}^{v} \mathbb{Z}_{+}\right)^{\oplus b_{k}(v)-\delta\left(v, a_{k}\right)}
$$

-where we write $\delta(i, j) \stackrel{\text { def }}{=} 1$ (respectively, $\stackrel{\text { def }}{=} 0)$ if $i=j$ (respectively, $i \neq j$ ).

REMARK 1.4.1. One verifies easily that the isomorphism class of the module $\mathbb{I}_{k}$ of Definition 1.4, (ii), depends only on $p_{k}, f_{k}, e_{k}$, and $a_{k}$.

Proposition 1.5. The module $\mathscr{I}_{k} /\left(\mathcal{O}_{k}\right)_{+}[c f$. [3], Lemma 1.2, (vi)] is isomorphic, as an abstract module, to the module $\mathbb{I}_{k}$. In particular, the isomorphism class of $\mathscr{I}_{k} /\left(\mathcal{O}_{k}\right)_{+}$depends only on $p_{k}, f_{k}, e_{k}$, and $a_{k}[c f$. Remark 1.4.1].

Proof. If $\left(\varepsilon_{k}, e_{k}\right)=(1,1)$, then Proposition 1.5 follows from Proposition 1.1, (ii), (v). Thus, we may assume without loss of generality that $\left(\varepsilon_{k}, e_{k}\right) \neq$ $(1,1)$. If $a_{k}^{[\delta]}=0$, then Proposition 1.5 follows immediately from [10], The- 
orem 2 [i.e., in the case where we take the " $(N, t)$ " of [10], Theorem 2 , to be $\left.\left(\varepsilon_{k} \cdot e_{k}-1,0\right)\right]$, together with Proposition 1.1, (iii); Lemma 1.3, (iv). If $a_{k}^{[\delta]}=1$, then Proposition 1.5 follows immediately from [10], Theorem 3 [i.e., in the case where we take the " $N$ " of [10], Theorem 3 , to be $\varepsilon_{k} \cdot e_{k}-1$ ], together with Proposition 1.1, (iii); Lemma 1.3, (i), (iv). This completes the proof of Proposition 1.5.

REMARK 1.5.1. One may give an alternative proof of Proposition 1.1, (ii), by applying Proposition 1.5. Indeed, it follows from conditions (1) and (2) of [3], Lemma 1.3, (i), that $\mu_{k}\left(\mathscr{I}_{k}\right)=\#\left(\mathscr{I}_{k} /\left(\mathscr{O}_{k}\right)_{+}\right)$. On the other hand, it follows from Proposition 1.5 that

$$
\begin{aligned}
\log _{p_{k}}\left(\#\left(\mathscr{I}_{k} /\left(\mathcal{O}_{k}\right)_{+}\right)\right) & =\log _{p_{k}}\left(\# \mathbb{I}_{k}\right)=\sum_{v=1}^{\infty}\left(v \cdot\left(b_{k}(v)-\delta\left(v, a_{k}\right)\right)\right) \\
& =\left\lfloor\frac{\varepsilon_{k} \cdot e_{k}-1}{p_{k}^{0}}\right\rfloor \cdot f_{k}-a_{k}=\varepsilon_{k} \cdot d_{k}-f_{k}-a_{k} .
\end{aligned}
$$

Thus, Proposition 1.1, (ii), holds.

LEMMA 1.6. The following hold:

(i) The $\mathbb{F}_{p_{k}}$-vector space $\left(\mathscr{I}_{k} /\left(\mathcal{O}_{k}\right)_{+}\right) \otimes_{\mathbb{Z}} \mathbb{F}_{p_{k}}$ is of dimension

$$
\varepsilon_{k} \cdot d_{k}-f_{k}-a_{k}^{[\delta]}-\left\lfloor\frac{\varepsilon_{k} \cdot e_{k}-1}{p_{k}}\right\rfloor \cdot f_{k} .
$$

(ii) If $p_{k}=2$, then the $\mathbb{F}_{p_{k}}$-vector space $\left(\mathscr{I}_{k} /\left(\mathcal{O}_{k}\right)_{+}\right) \otimes_{\mathbb{Z}} \mathbb{F}_{p_{k}}$ is of dimension $d_{k}-1$.

(iii) The $\mathbb{F}_{p_{k}}$-vector space $\left(\mathscr{I}_{k} /\left(\mathcal{O}_{k}\right)_{+}\right) \otimes_{\mathbb{Z}} \mathbb{F}_{p_{k}}$ is of dimension $<d_{k}$.

Proof. First, we verify assertion (i). It follows from Proposition 1.5, together with the definition of $\mathbb{I}_{k}$, that the dimension under consideration is given by

$$
\begin{aligned}
\sum_{v=1}^{\infty}\left(b_{k}(v)-\delta\left(v, a_{k}\right)\right) & =\left(\left\lfloor\frac{\varepsilon_{k} \cdot e_{k}-1}{p_{k}^{0}}\right\rfloor-\left\lfloor\frac{\varepsilon_{k} \cdot e_{k}-1}{p_{k}^{1}}\right\rfloor\right) \cdot f_{k}-a_{k}^{[\delta]} \\
& =\varepsilon_{k} \cdot d_{k}-f_{k}-a_{k}^{[\delta]}-\left\lfloor\frac{\varepsilon_{k} \cdot e_{k}-1}{p_{k}}\right\rfloor \cdot f_{k} .
\end{aligned}
$$

This completes the proof of assertion (i). Assertion (ii) follows from assertion (i), together with the [easily verified] fact that if $p_{k}=2$, then $\left(\varepsilon_{k}, a_{k}^{[\delta]}\right)=(2,1)$.

Finally, we verify assertion (iii). If $p_{k}$ is $o d d$, then since $\varepsilon_{k}=1, f_{k} \geq 1$, $e_{k} \geq 1$, and $a_{k}^{[\delta]} \geq 0$, assertion (iii) follows from assertion (i). If $p_{k}=2$, then 
assertion (iii) follows from assertion (ii). This completes the proof of assertion (iii), hence also of Lemma 1.6.

COROLLARY 1.7. It holds that

$$
\left(\mathcal{O}_{k}\right)_{+} \nsubseteq \frac{1}{2} \cdot \log _{k}\left(\mathcal{O}_{k}^{\times}\right)
$$

ProOF. Since $\mathscr{I}_{k}$ is given by $\left(2 p_{k}\right)^{-1} \cdot \log _{k}\left(\mathcal{O}_{k}^{\times}\right)$, it follows immediately from [3], Lemma 1.2, (vi), that it holds that $\left(\mathcal{O}_{k}\right)_{+}$is contained in $2^{-1} \cdot \log _{k}\left(\mathcal{O}_{k}^{\times}\right)$ if and only if $\operatorname{dim}_{\mathbb{F}_{p_{k}}}\left(\left(\mathscr{I}_{k} /\left(\mathcal{O}_{k}\right)_{+}\right) \otimes_{\mathbb{Z}} \mathbb{F}_{p_{k}}\right)$ is equal to $\operatorname{dim}_{\mathbb{F}_{p_{k}}}\left(\mathscr{I}_{k} \otimes_{\mathbb{Z}} \mathbb{F}_{p_{k}}\right)$, i.e., $d_{k}$. Thus, Corollary 1.7 follows from Lemma 1.6, (iii). This completes the proof of Corollary 1.7.

LEMMA 1.8. The following hold:

(i) The following four conditions are equivalent:

(1) The submodule $\mathscr{I}_{k} \subseteq k_{+}$coincides with the submodule $\left(\mathcal{O}_{k}\right)_{+} \subseteq k_{+}$.

(2) There exists $a(n)$ [necessarily nonpositive-cf. [3], Lemma 1.2, (vi)] integer $v$ such that the submodule $\mathscr{I}_{k} \subseteq k_{+}$coincides with the submodule $p_{k}^{v} \cdot\left(\mathcal{O}_{k}\right)_{+} \subseteq k_{+}$.

(3) It holds that $\varepsilon_{k} \cdot d_{k}=f_{k}+a_{k}$.

(4) One of the following three conditions is satisfied:

(a) It holds that $\left(\varepsilon_{k}, e_{k}\right)=(1,1)\left[i . e\right.$. , that the prime number $p_{k}$ is odd, and, moreover, $e_{k}=1$ ].

(b) It holds that $\left(p_{k}, f_{k}, e_{k}\right)=(2,1,1)$ [i.e., that $k$ is isomorphic to $\left.\mathbb{Q}_{2}\right]$.

(c) It holds that $\left(p_{k}, f_{k}, e_{k}, a_{k}\right)=(3,1,2,1)$ [i.e., that $k$ is isomorphic to $\mathbb{Q}_{3}\left(\zeta_{3}\right)$ - cf. Proposition 1.1, (iv)].

(ii) Suppose that either (a) or (b) in (i) is satisfied. Then, for each nonnegative integer $v$, it holds that $p_{k}^{v} \cdot \mathscr{I}_{k}=\mathrm{m}_{k}^{v}$.

(iii) Suppose that (c) in (i) is satisfied. Then, for each nonnegative integer $v$, it holds that $p_{k}^{v} \cdot \mathscr{I}_{k}=\mathrm{m}_{k}^{2 v}, p_{k}^{v-1} \cdot \mathrm{m}_{k}^{3}=\mathrm{m}_{k}^{2 v+1}$.

(iv) Suppose that (c) in (i) is satisfied. Write $K \stackrel{\text { def }}{=} k\left(\zeta_{9}\right) \subseteq \bar{k}$. Then the image of the composite

$$
\mathcal{O}_{K}^{\prec} \hookrightarrow \mathcal{O}_{K}^{\times} \stackrel{\mathrm{Nm}_{K / k}}{\longrightarrow} \mathcal{O}_{k}^{\times} \stackrel{\log _{k}}{\longrightarrow} k_{+}
$$

- where we write $\mathrm{Nm}_{K / k}$ for the Norm map with respect to the finite extension $K / k$-coincides with $\mathrm{m}_{k}^{3} \subseteq k_{+}$.

Proof. First, we verify assertion (i). The implication $(1) \Rightarrow(2)$ is immediate. Moreover, the equivalence $(1) \Leftrightarrow(3)$ follows from Proposition 1.1, (ii), and [3], Lemma 1.2, (vi). One also verifies immediately the implication (4) $\Rightarrow$ (3) by straightforward calculations [cf. also Proposition 1.1, (v)]. 
Next, we verify the implication $(2) \Rightarrow(1)$. Suppose that condition (2) is satisfied. Then since $\left(\mathcal{O}_{k}\right)_{+}$is a free $\mathbb{Z}_{p_{k}}$-module of rank $d_{k}$, we conclude that the module $\mathscr{I}_{k} /\left(\mathcal{O}_{k}\right)_{+}$is a free $\mathbb{Z} / p_{k}^{-v} \mathbb{Z}$-module of rank $d_{k}$. In particular, if $v \neq 0$, then the $\mathbb{F}_{p_{k}}$-vector space $\left(\mathscr{I}_{k} /\left(\mathcal{O}_{k}\right)_{+}\right) \otimes_{\mathbb{Z}} \mathbb{F}_{p_{k}}$ is of dimension $d_{k}$. Thus, it follows from Lemma 1.6, (iii), that $v=0$, as desired. This completes the proof of the implication $(2) \Rightarrow(1)$.

Finally, we verify the implication $(3) \Rightarrow(4)$. Suppose that condition (3) is satisfied. Then since $p_{k}^{a_{k}-1} \cdot\left(p_{k}-1\right) \leq e_{k}$ [cf. Proposition 1.1, (v)], we obtain that

$$
\varepsilon_{k} \cdot f_{k} \cdot p_{k}^{a_{k}-1} \cdot\left(p_{k}-1\right) \leq \varepsilon_{k} \cdot d_{k}=f_{k}+a_{k} .
$$

Now suppose that $p_{k}$ is $o d d$, i.e., $\geq 3$. Then we obtain that

$$
\left(3^{a_{k}-1} \cdot\left(p_{k}-1\right)-1\right) \cdot f_{k} \leq a_{k} .
$$

Thus, one verifies easily that either $\left(p_{k}, f_{k}, a_{k}\right)=(3,1,1)$ or $a_{k}=0$. Now observe that it follows from condition (3) that $\left(p_{k}, f_{k}, a_{k}\right)=(3,1,1)$ (respectively, $\left.a_{k}=0\right)$ implies that $\left(p_{k}, f_{k}, e_{k}, a_{k}\right)=(3,1,2,1)$ (respectively, $\left.e_{k}=1\right)$, as desired. This completes the proof of the implication $(3) \Rightarrow(4)$ in the case where $p_{k}$ is odd.

Next, suppose that $p_{k}=2$. Then, by the above inequality $\varepsilon_{k} \cdot f_{k} \cdot p_{k}^{a_{k}-1}$. $\left(p_{k}-1\right) \leq f_{k}+a_{k}$, we obtain that

$$
\left(2^{a_{k}}-1\right) \cdot f_{k} \leq a_{k},
$$

which thus implies that $a_{k}=1$. In particular, it follows from condition (3) that $2 d_{k}=f_{k}+1$, i.e., $f_{k} \cdot\left(2 e_{k}-1\right)=1$. Thus, we conclude that $\left(f_{k}, e_{k}\right)=$ $(1,1)$, as desired. This completes the proof of the implication $(3) \Rightarrow(4)$, hence also of assertion (i).

Assertions (ii), (iii) follow from the implication (4) $\Rightarrow(1)$ of assertion (i). Finally, we verify assertion (iv). Let us first observe that one verifies easily that the integer " $t$ " discussed in [14], Chapter V, $\S 3$, for the finite Galois extension $K / k$ [that is totally ramified and of degree 3] is equal to 2. Moreover, it follows from Proposition 1.1, (iv), that $f_{K}=1$.

Now since " $t$ " is equal to 2 , it follows from the second equality of [14], Chapter V, $\S$, Corollary 3, that $\operatorname{Nm}_{K / k}\left(\mathcal{O}_{K}^{\prec}\right)$ contains $\mathcal{O}_{k}^{\prec 3}$, which thus implies [cf. [11], Chapter II, Proposition 5.5] that

$$
\mathrm{m}_{k}^{3} \subseteq \log _{k}\left(\mathrm{Nm}_{K / k}\left(\mathcal{O}_{K}^{\prec}\right)\right) .
$$

Next, observe that since $f_{K}=1$, one verifies immediately from Lemma 1.3, (i), (ii), that $\mathcal{O}_{K}^{\prec}$ is generated by $\mathcal{O}_{K}^{\prec} \subseteq \mathcal{O}_{K}^{\prec}$ and $\zeta_{9} \in \mathcal{O}_{K}^{\prec}$. Thus, it follows from [3], 
Lemma 1.2, (v), that

$$
\log _{k}\left(\mathrm{Nm}_{K / k}\left(\mathcal{O}_{K}^{\prec}\right)\right)=\log _{k}\left(\mathrm{Nm}_{K / k}\left(\mathcal{O}_{K}^{\prec 2}\right)\right) .
$$

Next, observe that since " $t$ " is equal to 2 , and $f_{K}=1$, it follows immediately from [14], Chapter V, §3, Proposition 5, (iii), together with Lemma 1.3, (ii), that $\operatorname{Nm}_{K / k}\left(\mathcal{O}_{K}^{\prec 2}\right)$ is contained in $\mathcal{O}_{k}^{\prec 3}$, which thus implies [cf. [11], Chapter II, Proposition 5.5] that

$$
\log _{k}\left(\mathrm{Nm}_{K / k}\left(\mathcal{O}_{K}^{\prec 2}\right)\right) \subseteq \mathrm{m}_{k}^{3} .
$$

Thus, we conclude that $\mathrm{m}_{k}^{3}=\log _{k}\left(\mathrm{Nm}_{K / k}\left(\mathcal{O}_{K}^{\prec}\right)\right)$, as desired. This completes the proof of assertion (iv), hence also of Lemma 1.8 .

DEFINITION 1.9.

(i) We shall write

$v_{k}$

for the nonnegative integer defined as follows [cf. also Remark 1.9.1 below]:

(1) Suppose that either $\left(\varepsilon_{k}, e_{k}\right)=(1,1)$ or $\left(p_{k}, f_{k}, e_{k}, a_{k}\right) \in\{(2,1,1,1)$, $(3,1,2,1)\}$. Then

$$
v_{k} \stackrel{\text { def }}{=} 0 \text {. }
$$

(2) Suppose that the condition in (1) is not satisfied [which thus implies that $\left.\varepsilon_{k} \cdot e_{k}-1 \neq 0\right]$, and that either $p_{k} \geq 5$ or $k \neq \mathbb{Q}_{p_{k}}\left(\zeta_{p_{k}}\right)$. Then

$$
v_{k} \stackrel{\text { def }}{=} \max \left\{v \geq 0 \mid\left\lfloor\frac{\varepsilon_{k} \cdot e_{k}-1}{p_{k}^{v-1}}\right\rfloor \neq 0\right\} .
$$

(3) Suppose that the condition in (1) is not satisfied [which thus implies that $\varepsilon_{k} \cdot e_{k}-1 \neq 0$ ], that $p_{k} \leq 3$, and that $k \cong \mathbb{Q}_{p_{k}}\left(\zeta_{p_{k}}\right)$ [which thus implies that $\left.a_{k}^{[\delta]}=1\right]$. Then

$$
v_{k} \stackrel{\text { def }}{=} a_{k}-1,
$$

or, alternatively [cf. the proof of Proposition 1.10, (i), below],

$$
v_{k} \stackrel{\text { def }}{=} \max \left\{v \geq 0 \mid\left\lfloor\frac{\varepsilon_{k} \cdot e_{k}-1}{p_{k}^{v-1}}\right\rfloor \neq 0\right\}-1 .
$$

(ii) We shall write

$$
\operatorname{ord}_{k}^{[\mathscr{I}]}: k \backslash\{0\} \rightarrow \mathbb{Z}
$$

for the map of sets defined by

$$
\operatorname{ord}_{k}^{[\mathscr{I}]}(a) \stackrel{\text { def }}{=}-e_{k} \cdot \min \left\{v \in \mathbb{Z} \mid p_{k}^{v} \cdot a \in \mathscr{I}_{k}\right\}+e_{k}-1 .
$$


REMARK 1.9.1. One verifies easily that the nonnegative integer $v_{k}$ of Definition 1.9, (i), may be defined as follows:

(a) If either $p_{k} \geq 5$ or $k$ is not isomorphic to $\mathbb{Q}_{p_{k}}\left(\zeta_{p_{k}}\right)$, then

$$
v_{k} \stackrel{\text { def }}{=} \min \left\{v \geq 0 \mid \varepsilon_{k} \cdot e_{k} \leq p_{k}^{v}\right\} .
$$

(b) If $p_{k} \leq 3$, and $k$ is isomorphic to $\mathbb{Q}_{p_{k}}\left(\zeta_{p_{k}} a_{k}\right)$, then

$$
v_{k} \stackrel{\text { def }}{=} \min \left\{v \geq 0 \mid \varepsilon_{k} \cdot e_{k} \leq p_{k}^{v+1}\right\}=\min \left\{v \geq 1 \mid \varepsilon_{k} \cdot e_{k} \leq p_{k}^{v}\right\}-1 .
$$

Proposition 1.10. The following hold:

(i) The nonnegative integer $v_{k}$ is the smallest integer such that

$$
p_{k}^{v_{k}} \cdot \mathscr{I}_{k} \subseteq\left(\mathcal{O}_{k}\right)_{+} \subseteq \mathscr{I}_{k} .
$$

(ii) For each $a \in k \backslash\{0\}$, it holds that

$$
\operatorname{ord}_{k}(a) \leq \operatorname{ord}_{k}^{[\mathscr{I}]}(a)<\operatorname{ord}_{k}(a)+e_{k} \cdot\left(v_{k}+1\right) .
$$

Proof. First, we verify assertion (i). Assertion (i) in the case where the condition in (1) of Definition 1.9, (i), is satisfied follows from the implication $(4) \Rightarrow(1)$ of Lemma 1.8, (i). Thus, we may assume without loss of generality that the condition in (1) of Definition 1.9, (i), is not satisfied. [In particular, it holds that $\varepsilon_{k} \cdot e_{k}-1 \neq 0$.]

Write

$$
v_{\mathscr{I}}
$$

for the smallest integer such that $p_{k}^{v_{\mathscr{f}}} \cdot \mathscr{I}_{k} \subseteq\left(\mathcal{O}_{k}\right)_{+} \subseteq \mathscr{I}_{k}$ and

$$
v_{b} \stackrel{\text { def }}{=} \max \left\{v \geq 0 \mid b_{k}(v) \neq 0\right\} .
$$

Then it is immediate from Proposition 1.5 that

$$
v_{\mathscr{I}}=\max \left\{v \geq 0 \mid b_{k}(v)-\delta\left(v, a_{k}\right) \neq 0\right\} .
$$

In particular, we obtain the following two assertions:

(a) If $b_{k}\left(v_{b}\right) \neq \delta\left(v_{b}, a_{k}\right)$, then it holds that $v_{\mathscr{I}}=v_{b}$.

(b) If $b_{k}\left(v_{b}\right)=\delta\left(v_{b}, a_{k}\right)$ [or, alternative, $v_{b}=a_{k} \geq 1$ and $b_{k}\left(v_{b}\right)=1$ ], and $b_{k}\left(v_{b}-1\right) \neq 0$, then it holds that $v_{\mathscr{I}}=v_{b}-1$.

Moreover, let us observe that it follows immediately from the definition of $b_{k}(v)$ that

$$
v_{b}=\max \left\{v \geq 0\left\lfloor\mid \frac{\varepsilon_{k} \cdot e_{k}-1}{p_{k}^{v-1}}\right\rfloor \neq 0\right\} .
$$

Now we verify assertion (i) in the case where the condition in (2) of Definition 1.9, (i), is satisfied. Suppose that the condition in (2) of Definition 1.9, (i), is satisfied. Assume, moreover, that $b_{k}\left(v_{b}\right)=\delta\left(v_{b}, a_{k}\right)$ [which thus 
implies - cf. the above assertion (b) - that $v_{b}=a_{k} \geq 1$ and $\left.b_{k}\left(v_{b}\right)=1\right]$. Then one verifies immediately that

$$
v_{b}=a_{k} \geq 1, \quad f_{k}=1, \quad p_{k}^{v_{b}-1} \leq \varepsilon_{k} \cdot e_{k}-1<2 \cdot p_{k}^{v_{b}-1} .
$$

In particular, since $p_{k}^{a_{k}-1} \cdot\left(p_{k}-1\right) \leq e_{k}$ [cf. Proposition 1.1, (v)], we obtain that

$$
\varepsilon_{k} \cdot p_{k}^{a_{k}-1} \cdot\left(p_{k}-1\right)-1<2 \cdot p_{k}^{a_{k}-1},
$$

which thus implies that

$$
\varepsilon_{k} \cdot\left(p_{k}-1\right)-p_{k}^{1-a_{k}}<2 .
$$

Thus, since $a_{k} \geq 1$, we obtain that $p_{k} \leq 3$.

Next, let us observe that since $a_{k} \geq 1, f_{k}=1$, and $p_{k} \leq 3$, it follows immediately from the condition in (2) of Definition 1.9, (i), together with Proposition 1.1, (iv), (v), that

$$
2 \cdot p_{k}^{a_{k}-1} \cdot\left(p_{k}-1\right) \leq e_{k} .
$$

In particular, since $\varepsilon_{k} \cdot e_{k}-1<2 \cdot p_{k}^{v_{b}-1}$, we obtain that

$$
2 \cdot \varepsilon_{k} \cdot p_{k}^{a_{k}-1} \cdot\left(p_{k}-1\right)-1<2 \cdot p_{k}^{a_{k}-1},
$$

which thus implies that

$$
2 \cdot \varepsilon_{k} \cdot\left(p_{k}-1\right)-p_{k}^{1-a_{k}}<2 .
$$

Thus, since $a_{k} \geq 1$, we obtain a contradiction. In particular, we obtain that $b_{k}\left(v_{b}\right) \neq \delta\left(v_{b}, a_{k}\right)$, which thus implies [cf. the above assertion (a)] assertion (i) in the case where the condition in (2) of Definition 1.9, (i), is satisfied. This completes the proof of assertion (i) in the case where the condition in (2) of Definition 1.9, (i), is satisfied.

Finally, we verify assertion (i) in the case where the condition in (3) of Definition 1.9, (i), is satisfied. Suppose that the condition in (3) of Definition 1.9, (i), is satisfied. Then since $k$ is isomorphic to $\mathbb{Q}_{p_{k}}\left(\zeta_{p_{k}}\right)$, and $a_{k}^{[\delta]}=1$, it follows from Proposition 1.1, (iv), that $e_{k}=p_{k}^{a_{k}-1} \cdot\left(p_{k}-1\right)$. In particular, since $p_{k} \leq 3$, we obtain that

$$
\begin{aligned}
& \left\lfloor\frac{\varepsilon_{k} \cdot e_{k}-1}{p_{k}^{a_{k}}}\right\rfloor=\left\lfloor\frac{\varepsilon_{k} \cdot p_{k}^{a_{k}-1} \cdot\left(p_{k}-1\right)-1}{p_{k}^{a_{k}}}\right\rfloor=\left\lfloor\varepsilon_{k}-\frac{\varepsilon_{k}}{p_{k}}-\frac{1}{p_{k}^{a_{k}}}\right\rfloor=0, \\
& \left\lfloor\frac{\varepsilon_{k} \cdot e_{k}-1}{p_{k}^{a_{k}-1}}\right\rfloor=\left\lfloor\frac{\varepsilon_{k} \cdot p_{k}^{a_{k}-1} \cdot\left(p_{k}-1\right)-1}{p_{k}^{a_{k}-1}}\right\rfloor=\left\lfloor\varepsilon_{k} \cdot p_{k}-\varepsilon_{k}-\frac{1}{p_{k}^{a_{k}-1}}\right\rfloor=1, \\
& \left\lfloor\frac{\varepsilon_{k} \cdot e_{k}-1}{p_{k}^{a_{k}-2}}\right\rfloor=\left\lfloor\frac{\varepsilon_{k} \cdot p_{k}^{a_{k}-1} \cdot\left(p_{k}-1\right)-1}{p_{k}^{a_{k}-2}}\right\rfloor=\left\lfloor\varepsilon_{k} \cdot p_{k}^{2}-\varepsilon_{k} \cdot p_{k}-\frac{1}{p_{k}^{a_{k}-2}}\right\rfloor \geq 3 \text {. }
\end{aligned}
$$


Thus, since $f_{k}=1$ [cf. Proposition 1.1, (iv)], we conclude that

$$
v_{b}=a_{k} \geq 1, \quad b_{k}\left(v_{b}\right)=\delta\left(v_{b}, a_{k}\right), \quad b_{k}\left(v_{b}-1\right) \neq 0 .
$$

In particular, assertion (i) in the case where the condition in (3) of Definition 1.9, (i), is satisfied follows from the above assertion (b). This completes the proof of assertion (i) in the case where the condition in (3) of Definition 1.9, (i), is satisfied, hence also of assertion (i).

Next, we verify assertion (ii). Write $N \stackrel{\text { def }}{=}-\min \left\{v \in \mathbb{Z} \mid p_{k}^{v} \cdot a \in \mathscr{I}_{k}\right\}$. Then it follows from the definition of $N$ that $p_{k}^{-N} \cdot a \in \mathscr{I}_{k}$ but $p_{k}^{-N-1} \cdot a \notin \mathscr{I}_{k}$. Thus, it follows from assertion (i) that $p_{k}^{v_{k}-N} \cdot a \in p_{k}^{v_{k}} \cdot \mathscr{I}_{k} \subseteq\left(\mathcal{O}_{k}\right)_{+}$but $p_{k}^{-N-1} \cdot a \notin$ $\left(\mathcal{O}_{k}\right)_{+}$. In particular, we obtain that $\operatorname{ord}_{k}\left(p_{k}^{v_{k}-N} \cdot a\right) \geq 0$ and $\operatorname{ord}_{k}\left(p_{k}^{-N-1} \cdot a\right)<$ 0 , which thus implies that

$$
e_{k} \cdot\left(N-v_{k}\right) \leq \operatorname{ord}_{k}(a)<e_{k} \cdot(N+1) .
$$

Thus, it follows from the definition of $\operatorname{ord}_{k}^{[\mathscr{f}]}(a)$ that

$$
\operatorname{ord}_{k}^{[\mathscr{I}]}(a)-e_{k}+1-e_{k} \cdot v_{k} \leq \operatorname{ord}_{k}(a) \leq \operatorname{ord}_{k}^{[\mathscr{I}]}(a) .
$$

This completes the proof of assertion (ii), hence also of Proposition 1.10.

REMARK 1.10.1. By Proposition 1.10, (ii), one may regard the map $\operatorname{ord}_{k}^{[\mathscr{G}]}: k \backslash\{0\} \rightarrow \mathbb{Z}$ of Definition 1.9, (ii), as a sort of " $p_{k}$-adic valuation with an indeterminacy".

\section{Reconstruction algorithms related to valuations}

In the present $\S 2$, we maintain the notational conventions introduced at the beginning of the preceding $\S 1$. In particular, we have been given an $M L F$ $k$.

Moreover, let

\section{$G$}

be a [profinite - cf. [3], Proposition 3.3, (i)] group of MLF-type [cf. [3], Definition 3.1]. Thus, by applying the various group-theoretic reconstruction algorithms [cf. [8], Remark 1.9.8] of [3], §3, and [3], §4, to the group $G$ of MLF-type, we obtain

- a prime number $p(G)$,

- positive integers $d(G), f(G)$, and $e(G)$,

- subgroups $P(G) \subseteq I(G) \subseteq G$ of $G$,

- an element $\operatorname{Frob}(G) \in G / I(G)$ of $G / I(G)$, 
- topological monoids $\mathcal{O}^{\prec}(G) \subseteq \mathcal{O}^{\times}(G) \subseteq \mathcal{O}^{\triangleright}(G) \subseteq k^{\times}(G) \stackrel{\operatorname{rec}(G)}{\longrightarrow} G^{\mathrm{ab}}$,

- monoids $\underline{k}^{\times}(G) \subseteq \underline{k}_{\times}(G)$ and $k_{\times}(G)$,

- topological modules $\mathscr{I}(G) \subseteq k_{+}(G)$,

- a measure $\mu(G)$ on $k_{+}(G)$,

- $G$-monoids $\overline{\mathcal{O}}^{\times}(G) \subseteq \overline{\mathcal{O}}^{\triangleright}(G) \subseteq \bar{k}^{\times}(G) \subseteq \bar{k}_{\times}(G)$ and $\underline{\bar{k}}^{\times}(G) \subseteq \underline{\bar{k}}_{\times}(G)$,

- a $G$-module $\bar{k}_{+}(G)$,

- a $G$-module $\mu(G)$, and

- a topological $G$-module $\Lambda(G)$

[cf. [3], Summary 3.15; [3], Summary 4.3].

In the present $\S 2$, we establish group-theoretic reconstruction algorithms for constructing, from the group $G$ of MLF-type, a homomorphism of modules

$$
\operatorname{ord}_{\bigotimes}(G): k^{\times}(G) \rightarrow \mathbb{Z}_{+}
$$

which "corresponds" to the $p_{k}$-adic valuation $\operatorname{ord}_{k}: k \backslash\{0\} \rightarrow \mathbb{Z}$ [cf. Definition 2.2, Proposition 2.3 below] and a map of sets

$$
\operatorname{ord}_{\boxplus}(G): k_{+}(G) \backslash\{0\} \rightarrow \mathbb{Z}
$$

which "corresponds" to the map ord ${ }_{k}^{[\mathscr{I}]}: k \backslash\{0\} \rightarrow \mathbb{Z}$ of sets of Definition 1.9, (ii) [cf. Definition 2.6, (ii); Proposition 2.7, (ii), below], i.e., a sort of " $p_{k}$-adic valuation with an indeterminacy" [cf. Remark 1.10.1]. Moreover, we also establish group-theoretic reconstruction algorithms for constructing, from a group of MLF-type that satisfies an additional condition, topological submodules

$$
\text { " } \mathrm{m}^{n}(-) \subseteq \mathcal{O}_{+}(-) \subseteq k_{+}(-) "
$$

- where $n$ is a nonnegative integer - of " $k_{+}(-)$" which "correspond" to the topological submodules $\mathrm{m}_{k}^{n} \subseteq\left(\mathcal{O}_{k}\right)_{+} \subseteq k_{+}$of $k_{+}$, respectively [cf. Definition 2.9, (i), (ii); Proposition 2.10 below].

Lemma 2.1. The module $k^{\times}(G) / \mathcal{O}^{\times}(G)$ is torsion-free and generated $b y$ $\operatorname{Frob}(G) \in k^{\times}(G) / \mathcal{O}^{\times}(G)(\subseteq G / I(G))$.

Proof. This assertion follows - in light of [3], Proposition 3.6; [3], Proposition 3.9; [3], Proposition 3.11, (i)—from [3], Lemma 1.5, (i), and [3], Lemma $1.7,(1)$.

DEFINITION 2.2. We shall write

$$
\operatorname{ord}_{\bigotimes}(G): k^{\times}(G) \rightarrow \mathbb{Z}
$$

for the map defined as follows [cf. [2], Theorem 1.4, (7)]: For each $a \in k^{\times}(G)$, write $\operatorname{ord}_{\bigotimes}(G)(a) \in \mathbb{Z}$ for the uniquely determined [cf. Lemma 2.1] integer $n$ such that the image of $a \in k^{\times}(G)$ in $k^{\times}(G) / \mathcal{O}^{\times}(G)$ coincides with $\operatorname{Frob}(G)^{n} \in$ $k^{\times}(G) / \mathcal{O}^{\times}(G)$. 
One verifies immediately that this map is, in fact, a homomorphism $k^{\times}(G) \rightarrow \mathbb{Z}_{+}$of modules.

Proposition 2.3. The vertical isomorphism $k^{\times} \stackrel{\sim}{\rightarrow} k^{\times}\left(G_{k}\right)$ in the diagram of [3], Proposition 3.11, (i), fits into a commutative diagram of modules

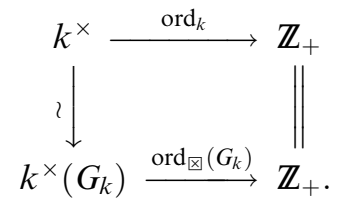

Proof. This assertion follows - in light of [3], Proposition 3.6; [3], Proposition 3.9; [3], Proposition 3.11, (i)—from Lemma 1.2.

REMARK 2.3.1. Let us observe that one verifies immediately from Proposition 2.3 that

- the open subsets of the topological module $k^{\times}(G)\left(\subseteq k_{\times}(G)\right)$ and,

- for each positive integer $n$, the subsets of $k_{\times}(G)$

$$
\left\{a \in k^{\times}(G) \mid \operatorname{ord}_{\bigotimes}(G)(a) \geq n\right\} \cup\left\{*_{k^{\times}(G)}\right\} \subseteq k_{\times}(G)
$$

generate a topology on the underlying set of the monoid $k_{\times}(G)$ by means of which one may regard $k_{\times}(G)$ as a topological monoid. Moreover, one also verifies immediately from Proposition 2.3 that the isomorphism $k_{\times} \stackrel{\sim}{\rightarrow} k_{\times}\left(G_{k}\right)$ of [3], Proposition 3.11, (ii), is an isomorphism of topological monoids.

DEFINITION 2.4 .

(i) We shall write

$$
\varepsilon(G) \stackrel{\text { def }}{=} \begin{cases}1 & \text { if } p(G) \neq 2 \\ 2 & \text { if } p(G)=2\end{cases}
$$

[cf. [3], Definition 3.13].

(ii) We shall write

$$
a(G) \stackrel{\text { def }}{=} \log _{p(G)}\left(\#\left(\left(k^{\times}(G)_{\text {tor }}\right)^{(p(G))}\right)\right)
$$

[cf. [3], Lemma 1.2, (i); [3], Proposition 3.11, (i)].

(iii) Let $v$ be a positive integer. Then we shall write

$$
\begin{gathered}
b(G, v) \stackrel{\text { def }}{=}\left(\left\lfloor\frac{\varepsilon(G) \cdot e(G)-1}{\left.p(G)^{v-1}\right\rfloor-2 \cdot\left\lfloor\frac{\varepsilon(G) \cdot e(G)-1}{p(G)^{v}}\right\rfloor}\right.\right. \\
\left.+\left\lfloor\frac{\varepsilon(G) \cdot e(G)-1}{p(G)^{v+1}}\right\rfloor\right) \cdot f(G) .
\end{gathered}
$$


(iv) We shall write

$$
\mathbb{I}(G) \stackrel{\text { def }}{=} \prod_{v=1}^{\infty}\left(\mathbb{Z}_{+} / p(G)^{v} \mathbb{Z}_{+}\right)^{\oplus b(G, v)-\delta(v, a(G))}
$$

-where we write $\delta(i, j) \stackrel{\text { def }}{=} 1$ (respectively, $\stackrel{\text { def }}{=} 0$ ) if $i=j$ (respectively, $i \neq j$ ).

Proposition 2.5. The following hold:

(i) It holds that

$$
\varepsilon_{k}=\varepsilon\left(G_{k}\right), \quad a_{k}=a\left(G_{k}\right) .
$$

(ii) The module $\mathscr{I}_{k} /\left(\mathcal{O}_{k}\right)_{+}$is isomorphic, as an abstract module, to the module $\mathbb{I}\left(G_{k}\right)$.

Proof. Assertion (i) follows from [3], Proposition 3.6, and [3], Proposition 3.11, (i). Assertion (ii) follows - in light of assertion (i); [3], Proposition 3.6-from Proposition 1.5. This completes the proof of Proposition 2.5.

DEFINITION 2.6.

(i) We shall write

$$
v(G)
$$

for the nonnegative integer defined as follows:

(1) If either $p(G) \geq 5$ or $(f(G), e(G)) \neq\left(1, p(G)^{a(G)-1} \cdot(p(G)-1)\right)$, then

$$
v(G) \stackrel{\text { def }}{=} \min \left\{v \geq 0 \mid \varepsilon(G) \cdot e(G) \leq p(G)^{v}\right\} .
$$

(2) If $p(G) \leq 3$ and $(f(G), e(G))=\left(1, p(G)^{a(G)-1} \cdot(p(G)-1)\right)$, then

$$
v(G) \stackrel{\text { def }}{=} \min \left\{v \geq 0 \mid \varepsilon(G) \cdot e(G) \leq p(G)^{v+1}\right\} .
$$

(ii) We shall write

$$
\operatorname{ord}_{\boxplus}(G): k_{+}(G) \backslash\{0\} \rightarrow \mathbb{Z}
$$

for the map of sets defined by

$$
\operatorname{ord}_{\boxplus}(G)(a) \stackrel{\text { def }}{=}-e(G) \cdot \min \left\{v \in \mathbb{Z} \mid p(G)^{v} \cdot a \in \mathscr{I}(G)\right\}+e(G)-1 .
$$

Proposition 2.7. The following hold:

(i) It holds that

$$
v_{k}=v\left(G_{k}\right)
$$


(ii) The vertical isomorphism $k_{+} \stackrel{\sim}{\rightarrow} k_{+}\left(G_{k}\right)$ in the diagram of [3], Proposition 3.11, (iv), fits into a commutative diagram of sets

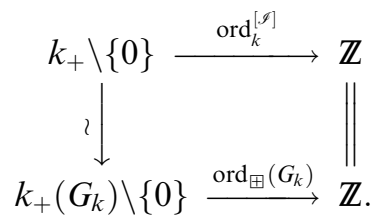

(iii) For each $a \in k \backslash\{0\}$, it holds that

$$
\operatorname{ord}_{k}(a) \leq \operatorname{ord}_{\boxplus}\left(G_{k}\right)(a)<\operatorname{ord}_{k}(a)+e\left(G_{k}\right) \cdot\left(v\left(G_{k}\right)+1\right) .
$$

Proof. Assertion (i) follows from Proposition 2.5, (i), and [3], Proposition 3.6, together with Proposition 1.1, (iv) [cf. also Remark 1.9.1]. Assertion (ii) follows from [3], Proposition 3.6, and [3], Proposition 3.11, (iv). Assertion (iii) follows - in light of assertions (i), (ii); [3], Proposition 3.6-from Proposition 1.10, (ii). This completes the proof of Proposition 2.7.

LEMMA 2.8. The following two conditions are equivalent:

(1) It holds that $\varepsilon(G) \cdot d(G)=f(G)+a(G)$.

(2) One of the following three conditions is satisfied:

(a) It holds that $(\varepsilon(G), e(G))=(1,1)$.

(b) It holds that $(p(G), f(G), e(G))=(2,1,1)$.

(c) It holds that $(p(G), f(G), e(G), a(G))=(3,1,2,1)$.

Proof. This assertion follows - in light of Proposition 2.5, (i); [3], Proposition 3.6 - from the equivalence $(3) \Leftrightarrow(4)$ of Lemma 1.8, (i).

Definition 2.9. Suppose that $\varepsilon(G) \cdot d(G)=f(G)+a(G)$.

(i) We shall write

$$
\mathcal{O}_{+}(G) \stackrel{\text { def }}{=} \mathscr{I}(G) \subseteq k_{+}(G)
$$

(ii) Let $n$ be a nonnegative integer. Then we shall define a topological submodule

$$
\mathrm{m}^{n}(G) \subseteq \mathcal{O}_{+}(G)
$$

of $\mathcal{O}_{+}(G)$ as follows:

(1) Suppose that either $(\varepsilon(G), e(G))=(1,1)$ or $(p(G), f(G), e(G))=$ $(2,1,1)$ [cf. Lemma 2.8]. Then we shall write

$$
\mathrm{m}^{n}(G) \stackrel{\text { def }}{=} p(G)^{n} \cdot \mathcal{O}_{+}(G) \subseteq \mathcal{O}_{+}(G) .
$$


(2) Suppose that $(p(G), f(G), e(G), a(G))=(3,1,2,1)$ [cf. Lemma 2.8]. If $n$ is even, then we shall write

$$
\mathrm{m}^{n}(G) \stackrel{\text { def }}{=} p(G)^{n / 2} \cdot \mathcal{O}_{+}(G) .
$$

If $n$ is odd, then we shall write

$$
\mathrm{m}^{n}(G) \stackrel{\text { def }}{=} p(G)^{(n-3) / 2} \cdot \operatorname{Im}\left(\mathcal{O}^{\prec}(H) \hookrightarrow \mathcal{O}^{\times}(H) \rightarrow \mathcal{O}^{\times}(G) \rightarrow k_{+}(G)\right)
$$

-where we write $H \subseteq G$ for the kernel of the natural action of $G$ on $\boldsymbol{\mu}(G)[9]$ $(\subseteq \boldsymbol{\mu}(G))$; the first arrow " $\hookrightarrow$ " is the natural inclusion; the second arrow " $\rightarrow$ " is the homomorphism induced by the homomorphism $H^{\mathrm{ab}} \rightarrow G^{\mathrm{ab}}$ determined by the inclusion $H \hookrightarrow G$; the third arrow " $\rightarrow$ " is the natural homomorphism.

Proposition 2.10. Suppose that $\varepsilon_{k} \cdot e_{k}=f_{k}+a_{k}$, or, alternatively [cf. Proposition 2.5, (i); [3], Proposition 3.6], that $\varepsilon\left(G_{k}\right) \cdot e\left(G_{k}\right)=f\left(G_{k}\right)+a\left(G_{k}\right)$. Let $n$ be a nonnegative integer. Then the vertical isomorphism $k_{+} \stackrel{\sim}{\rightarrow} k_{+}\left(G_{k}\right)$ in the diagram of [3], Proposition 3.11, (iv), fits into a commutative diagram of topological modules

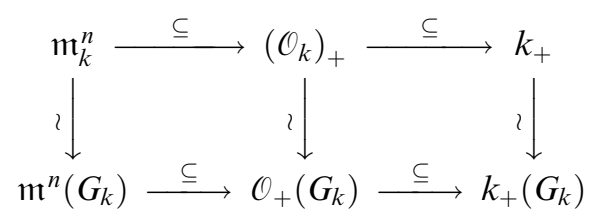

- where the horizontal arrows are the natural inclusions, and the vertical arrows are isomorphisms.

Proof. This assertion follows - in light of Proposition 2.5, (i); [3], Lemma 1.7, (2); [3], Proposition 3.6; [3], Proposition 3.11, (i), (iv)—from Lemma 1.8, (i), (ii), (iii), (iv).

Some of the group-theoretic reconstruction algorithms discussed in the present $\S 2$ may be summarized as follows.

SUMMARY 2.11 .

(i) There exist group-theoretic reconstruction algorithms [cf. [8], Remark 1.9.8] for constructing, from a group $G$ of MLF-type,

- nonnegative integers $\varepsilon(G), a(G)$, and $v(G)$ [cf. Definition 2.4, (i), (ii); Definition 2.6, (i)],

- a module $\mathbb{I}(G)[c f$. Definition 2.4, (iv)], 2.2], and

- a homomorphism $\operatorname{ord}_{凶}(G): k^{\times}(G) \rightarrow \mathbb{Z}_{+}$of modules $[$cf. Definition

- a map $\operatorname{ord}_{\boxplus}(G): k_{+}(G) \backslash\{0\} \rightarrow \mathbb{Z}$ of sets [cf. Definition 2.6, (ii)] which "correspond" to 
- the nonnegative integers $\varepsilon_{k}$, $a_{k}$, and $v_{k}$ [cf. Proposition 2.5, (i); Proposition 2.7, (i)],

- the quotient of $\mathscr{I}_{k}$ by $\left(\mathcal{O}_{k}\right)_{+}$[cf. Proposition 2.5, (ii)],

- the $p_{k}$-adic valuation $\operatorname{ord}_{k}: k \backslash\{0\} \rightarrow \mathbb{Z} \quad[c f$. Proposition 2.3],

and

- the " $p_{k}$-adic valuation with an indeterminacy" [cf. Remark 1.10.1] $\operatorname{ord}_{k}^{[\mathscr{f}]}: k \backslash\{0\} \rightarrow \mathbb{Z}[$ cf. Proposition 2.7, (ii)], respectively.

(ii) There exist group-theoretic reconstruction algorithms for constructing, from a group $G$ of MLF-type such that $\varepsilon(G) \cdot d(G)=f(G)+a(G)$, 2.9, (i)] and,

- a topological submodule $\mathcal{O}_{+}(G) \subseteq k_{+}(G)$ of $k_{+}(G)$ [cf. Definition

- for each nonnegative integer $n$, a topological submodule $\mathrm{m}^{n}(G) \subseteq$ $\mathcal{O}_{+}(G)$ of $\mathcal{O}_{+}(G)[c f$. Definition 2.9, (ii)]

which "correspond" to

and,

- the topological submodule $\left(\mathcal{O}_{k}\right)_{+} \subseteq k_{+}$of $k_{+}[c f$. Proposition 2.10]

- for each nonnegative integer $n$, the topological submodule $\mathrm{m}_{k}^{n} \subseteq\left(\mathcal{O}_{k}\right)_{+}$of $\left(\mathcal{O}_{k}\right)_{+}[$cf. Proposition 2.10],

respectively.

REMARK 2.11.1. Let us recall that, as asserted in Summary 2.11, (i), we have established [cf. Definition 2.6, (ii)] a group-theoretic reconstruction algorithm for constructing, from a group $G$ of MLF-type, a map $\operatorname{ord}_{\boxplus}(G)$ : $k_{+}(G) \backslash\{0\} \rightarrow \mathbb{Z}$ of sets which "corresponds" to the " $p_{k}$-adic valuation with an indeterminacy" $\operatorname{ord}_{k}^{[\mathscr{f}]}: k \backslash\{0\} \rightarrow \mathbb{Z}$ [cf. Remark 1.10.1].

Here, let us also recall that, as discussed in [3], Remark 4.3.1, (i) [cf. also [3], Remark 4.3.2], it is impossible to establish a group-theoretic reconstruction algorithm for constructing, from a group $G$ of MLF-type, a topology on the module $\bar{k}_{+}(G)$ which "corresponds" to the $p_{k}$-adic topology on the module $\bar{k}_{+}$. In particular, it is impossible to establish a group-theoretic reconstruction algorithm for constructing, from an arbitrary group $G$ of MLF-type, a map $k_{+}(G) \backslash\{0\} \rightarrow \mathbb{Z}$ of sets which "corresponds" to the $p_{k}$-adic valuation $k \backslash\{0\} \rightarrow$ $\mathbb{Z}$ [i.e., without any indeterminacy].

REMARK 2.11.2. Let us recall that, as asserted in Summary 2.11, (ii), we have established [cf. Definition 2.9, (i)] a group-theoretic reconstruction algorithm for constructing, from a group $G$ of MLF-type such that $\varepsilon(G) \cdot d(G)=$ $f(G)+a(G)$, a topological submodule $\mathcal{O}_{+}(G) \subseteq k_{+}(G)$ of $k_{+}(G)$ which "corresponds" to the topological submodule $\left(\mathcal{O}_{k}\right)_{+} \subseteq k_{+}$of $k_{+}$.

Here, let us also recall that, as discussed in [3], Remark 4.3.1, (iii) [cf. also [2], Remark 1.4.3], it is impossible to establish a group-theoretic reconstruction 
algorithm for constructing, from an arbitrary group $G$ of MLF-type, such a topological submodule of $k_{+}(G)$.

REMARK 2.11.3. Let us recall that, as asserted in Summary 2.11, (i), and [3], Summary 3.15, we have established [cf. Definition 2.4, (iv); [3], Definition 3.10, (vi)] group-theoretic reconstruction algorithms for constructing, from a group $G$ of MLF-type, modules $\mathscr{I}(G)$ and $\mathbb{I}(G)$ which "correspond" to the logshell $\mathscr{I}_{k}$ and the quotient $\mathscr{I}_{k} /\left(\mathcal{O}_{k}\right)_{+}$, respectively.

Here, let us also recall that, as discussed in [3], Remark 4.3.1, (iii) [cf. also [2], Remark 1.4.3], it is impossible to establish a group-theoretic reconstruction algorithm for constructing, from an arbitrary group $G$ of MLF-type, a surjection $\mathscr{I}(G) \rightarrow \mathbb{I}(G)$ which "corresponds" to the natural surjection $\mathscr{I}_{k} \rightarrow$ $\mathscr{I}_{k} /\left(\mathcal{O}_{k}\right)_{+}$.

\section{Open homomorphisms between profinite groups of MLF-type}

In the present $\S 3$, we maintain the notational conventions introduced at the beginnings of $\S 1$ and $\S 2$. In particular, we have been given a group of MLF-type

$G$.

In the present $\S 3$, we consider open homomorphisms between profinite groups of MLF-type. As a consequence of the results in the present $\S 3$, we prove that every open homomorphism between profinite groups of MLF-type such that the positive integer " $e(-)$ " [cf. the notational conventions introduced at the beginning of the preceding $\S 2]$ of the domain is equal to the positive integer " $e(-)$ " of the codomain is injective [cf. Corollary 3.7 below].

LEMMA 3.1. The following hold:

(i) The topological module $\left(G^{(p(G))}\right)^{\mathrm{ab} / \mathrm{tor}}$ is a free $\mathbb{Z}_{p(G)}$-module of rank $\boldsymbol{d}(\boldsymbol{G})+\mathbf{1}$. Moreover, the kernel of the natural homomorphism $\left(G^{(p(G))}\right)^{\mathrm{ab}} \rightarrow$ $\left(G^{(p(G))}\right)^{\mathrm{ab} / \text { tor }}$ is cyclic.

(ii) The closed subgroup $I(G) / P(G) \subseteq G / P(G)$ of $G / P(G)$ coincides with the kernel of the natural surjection $G / P(G) \rightarrow(G / P(G))^{\mathrm{ab} / \mathrm{tor}}$.

(iii) It holds that

$$
f(G)=\log _{p(G)}\left(1+\#\left((G / P(G))^{\text {ab-tor }}\right)\right) .
$$

Proof. Assertion (i) follows immediately - in light of [3], Proposition 3.6; the isomorphism in the final display of [3], Lemma 1.7, (1)-from [3], Lemma 1.2, (i). Assertions (ii), (iii) follow immediately - in light of [3], Proposition 3.6; [3], Proposition 3.9-from [3], Lemma 1.5, (i), (ii), (iii). This completes the proof of Lemma 3.1 . 
Definition 3.2. Let $J$ be a profinite group. Then we shall say that a closed subgroup $N \subseteq J$ of $J$ is quasi-normal [i.e., in $J$ ] if $N$ is normal in an open subgroup of $J$ that contains $N$.

REMARK 3.2.1. Let $J$ be a profinite group and $N \subseteq J$ a quasi-normal closed subgroup of $J$. Then one verifies easily that, for each closed subgroup $J_{1} \subseteq J$ of $J$, the closed subgroup $N \cap J_{1} \subseteq J_{1}$ of $J_{1}$ is quasi-normal.

Lemma 3.3. Let $J \subseteq G$ be a nontrivial closed subgroup of $G$. Then the following hold:

(i) Suppose that $J$ is quasi-normal in $G$. Then one of the following three conditions is satisfied [cf. also Remark 3.3.1 below]:

(1) The image of $J$ in $G^{(p(G))}$ is open.

(2) The maximal pro-p $(G)$ quotient $J^{(p(G))}$ is not topologically finitely generated.

(3) There is no nontrivial pro-p $(G)$ quotient of $J$.

(ii) Suppose that $J$ is quasi-normal in $G$. Then there exists an open subgroup of $J$ that has a nontrivial pro-p $(G)$ quotient.

(iii) Suppose that the maximal pro-p $(G)$ quotient $J^{(p(G))}$ is not procyclic. Then there exists an open subgroup $H \subseteq G$ of $G$ such that $J \subseteq H$, and, moreover, the image of $J$ in $\left(H^{(p(G))}\right)^{\mathrm{ab} / \mathrm{tor}}$ is nontrivial [hence also infinite].

(iv) Suppose that $J$ is quasi-normal in $G$. Then the following two conditions are equivalent:

(a) There is a nontrivial pro-p $(G)$ quotient of $J$.

(b) There exists an open subgroup $H \subseteq G$ of $G$ such that $J \subseteq H$, and, moreover, the image of $J$ in $\left(H^{(p(G))}\right)^{\text {ab/tor }}$ is nontrivial [hence also infinite].

Proof. First, we verify assertion (i). Let us first observe that, to verify assertion (i), it suffices to verify that if $J$ satisfies neither condition (1) nor condition (3), then $J$ satisfies condition (2). Suppose that $J$ satisfies neither condition (1) nor condition (3).

To verify that $J$ satisfies condition (2), let us observe that since $J$ does not satisfy condition (3), there exists a normal open subgroup $N \subseteq G$ of $G$ such that $J /(J \cap N)$ has a quotient that is a nontrivial $p(G)$-group. Thus, by considering the composite $J \hookrightarrow J \cdot N \rightarrow(J \cdot N) / N$ [that determines an isomorphism $J /(J \cap N) \stackrel{\sim}{\rightarrow}(J \cdot N) / N]$, we conclude that the image of $J$ in $(J \cdot N)^{(p(G))}$ is nontrivial. Next, since $J$ does not satisfy condition (1), the image of $J$ in $(J \cdot N)^{(p(G))}$ is not open. Thus, it follows immediately-in light of [3], Proposition 3.6-from [7], Theorem 1.7, (ii) [cf. also Remark 3.2.1], that the image of $J$ in $(J \cdot N)^{(p(G))}$ is not topologically finitely generated, which thus 
implies that $J$ satisfies condition (2), as desired. This completes the proof of assertion (i).

Assertion (ii) follows immediately - in light of [3], Proposition 3.6-from [1], Lemma 2.3. Next, we verify assertion (iii). Let us first observe that, by our assumption, there exists a normal open subgroup $N \subseteq G$ of $G$ such that $J /(J \cap N)$ has a quotient that is a noncyclic $p(G)$-group. Write $H \stackrel{\text { def }}{=} J \cdot N \subseteq$ $G$. Now let us recall the easily verified fact that, for a given $p(G)$-group, it holds that the $p(G)$-group is cyclic if and only if the abelianization of the $p(G)$-group is cyclic. Thus, by considering the composite $J \hookrightarrow H \rightarrow H / N$ [that determines an isomorphism $J /(J \cap N) \stackrel{\sim}{\rightarrow} H / N$ ], we conclude immediately that the image $\operatorname{Im}(J) \subseteq\left(H^{(p(G))}\right)^{\text {ab }}$ of $J$ in $\left(H^{(p(G))}\right)^{\text {ab }}$ is not cyclic. In particular, it follows immediately from Lemma 3.1, (i), that the image of $\operatorname{Im}(J) \subseteq$ $\left(H^{(p(G))}\right)^{\text {ab }}$ in $\left(H^{(p(G))}\right)^{\text {ab/tor }}$ is nontrivial. This completes the proof of assertion (iii).

Finally, we verify assertion (iv). The implication (b) $\Rightarrow(a)$ is immediate. Next, we verify the implication $(a) \Rightarrow(b)$. Suppose that the condition (a) is satisfied. If condition (1) of assertion (i) is satisfied, then the condition (b) is immediate. On the other hand, if condition (2) of assertion (i) is satisfied, then the condition (b) follows from assertion (iii). This completes the proof of assertion (iv), hence also of Lemma 3.3.

REMARK 3.3.1. Let us give an example that satisfies each of the three conditions in Lemma 3.3, (i):

(i) One verifies easily that $G$ itself satisfies condition (1) of Lemma 3.3, (i), i.e., that condition (1) of Lemma 3.3, (i), in the case where we take the " $J$ " to be $G$ is always satisfied.

(ii) Next, let us verify that condition (2) of Lemma 3.3, (i), in the case where we take the " $J$ " to be the normal closed subgroup $P(G) \subseteq G$ of $G$ is always satisfied. Indeed, this follows from [12], Proposition 7.5.1, together with [3], Proposition 3.6.

(iii) Finally, one verifies easily that condition (3) of Lemma 3.3, (i), in the case where we take the " $J$ " to be the kernel of the natural surjection $G \rightarrow G^{(p(G))}$ is always satisfied [cf. also [3], Lemma 1.5, (i)].

Proposition 3.4. For each $\square \in\{0, \bullet\}$, let $G_{\square}$ be a profinite group of MLF-type. Let

$$
\alpha: G_{\circ} \rightarrow G_{\bullet}
$$

be an open homomorphism. Then the following hold:

(i) The open homomorphism $\alpha$ fits into a commutative diagram of profinite groups 


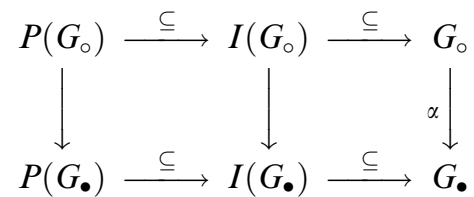

- where the horizontal arrows are the natural inclusions, and the vertical arrows are open. If, moreover, $\alpha$ is surjective, then the vertical arrows are surjective.

(ii) In the resulting $[c f$. (i)] commutative diagram of profinite groups

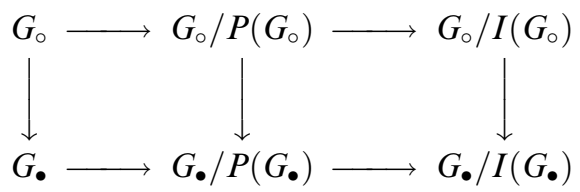

- where the horizontal arrows are the natural surjections - the middle and righthand vertical arrows are open injections. In particular, if, moreover, $\alpha$ is surjective, then the middle and right-hand vertical arrows are isomorphisms.

(iii) It holds that

$$
p\left(G_{\circ}\right)=p\left(G_{\bullet}\right), \quad d\left(G_{\circ}\right) \geq d\left(G_{\bullet}\right), \quad f\left(G_{\circ}\right) \in f\left(G_{\bullet}\right) \mathbb{Z}, \quad e\left(G_{\circ}\right) \geq e\left(G_{\bullet}\right) .
$$

If, moreover, $\alpha$ is surjective, then

$$
f\left(G_{\circ}\right)=f\left(G_{\bullet}\right) .
$$

(iv) The right-hand vertical arrow of the diagram of (ii) maps $\operatorname{Frob}\left(G_{\circ}\right) \in$ $G_{\circ} / I\left(G_{\circ}\right)$ to $\operatorname{Frob}\left(G_{\bullet}\right)^{f\left(G_{\circ}\right) / f\left(G_{\bullet}\right)} \in G_{\bullet} / I\left(G_{\bullet}\right)[c f$. (iii)]. In particular, if, moreover, $\alpha$ is surjective, then the right-hand vertical arrow of the diagram of (ii) maps $\operatorname{Frob}\left(G_{\circ}\right) \in G_{\circ} / I\left(G_{\circ}\right)$ to $\operatorname{Frob}\left(G_{\bullet}\right) \in G_{\bullet} / I\left(G_{\bullet}\right)[c f$. (iii) $]$.

Proof. Let us first observe that it follows immediately from [3], Proposition 3.6, and [3], Proposition 3.9, that, to verify Proposition 3.4, we may assume without loss of generality, by replacing $G_{\bullet}$. by the image of $\alpha$ [which is of MLF-type - cf. the discussion following [3], Proposition 3.3], that $\alpha$ is surjective.

First, we verify assertions (i), (ii). The assertion that $\alpha$ restricts to a surjection $P\left(G_{\circ}\right) \rightarrow P\left(G_{\bullet}\right)$, as well as the assertion that the resulting homomorphism $G_{\circ} / P\left(G_{\circ}\right) \rightarrow G_{\bullet} / P\left(G_{\bullet}\right)$ is an isomorphism, follows immediatelyin light of [3], Proposition 3.6-from [7], Proposition 3.4. In particular, the assertion that $\alpha$ restricts to a surjection $I\left(G_{\circ}\right) \rightarrow I\left(G_{\bullet}\right)$, as well as the assertion that the resulting homomorphism $G_{\circ} / I\left(G_{\circ}\right) \rightarrow G_{\bullet} / I\left(G_{\bullet}\right)$ is an isomorphism, follows immediately from Lemma 3.1, (ii). This completes the proofs of assertions (i), (ii).

Next, we verify assertion (iii). Let us first observe that the surjection $\alpha$ induces a surjection $G_{\circ}^{\mathrm{ab} / \text { tor }} /\left(p\left(G_{\bullet}\right) \cdot G_{\circ}^{\mathrm{ab} / \text { tor }}\right) \rightarrow G_{\bullet}^{\mathrm{ab} / \text { tor }} /\left(p\left(G_{\bullet}\right) \cdot G_{\bullet}^{\mathrm{ab} / \text { tor }}\right)$. Thus, 
it holds that $p\left(G_{\circ}\right)=p\left(G_{\bullet}\right)$ and $d\left(G_{\circ}\right) \geq d\left(G_{\bullet}\right)$. In particular, it followsin light of assertion (ii)-from Lemma 3.1, (iii), that $f\left(G_{\circ}\right)=f\left(G_{\bullet}\right)$, which thus implies that $e\left(G_{\circ}\right) \geq e\left(G_{\bullet}\right)$. This completes the proof of assertion (iii). Assertion (iv) follows from assertions (ii), (iii). This completes the proof of Proposition 3.4 .

Proposition 3.5. In the situation of Proposition 3.4, write $H_{\bullet} \subseteq G_{\bullet}$ for the image of $\alpha$ [which is of MLF-type - cf. the discussion following [3], Proposition 3.3]:

$$
\alpha: G_{\circ} \rightarrow H_{\bullet} \hookrightarrow G_{\bullet}
$$

Then the following hold:

(i) The open homomorphism $\alpha$ determines a commutative diagram of topological monoids

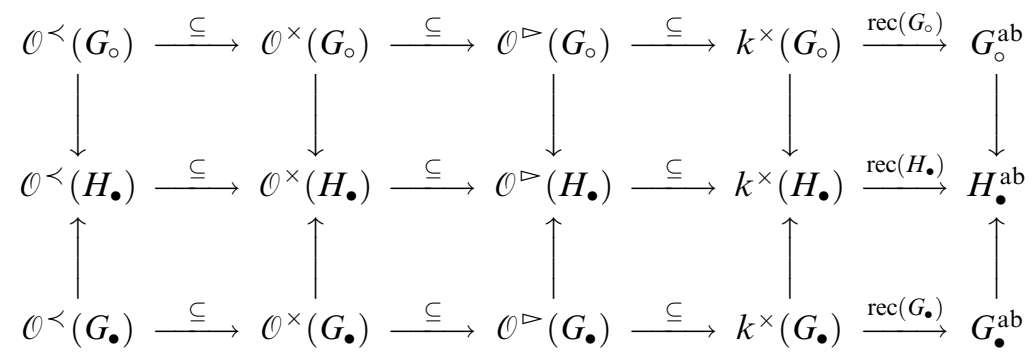

- where the horizontal arrows are the natural inclusions, the upper vertical arrows are the surjections induced by $\alpha$, and the lower vertical arrows are the injections determined by the transfer map [i.e., with respect to $\left.H_{\bullet} \subseteq G_{\bullet}\right]$ [cf. [3], Lemma 1.7, (3)].

(ii) The left-hand upper and left-hand lower squares of the diagram of (i) determine homomorphisms of modules

$$
\underline{k}^{\times}\left(G_{\circ}\right) \stackrel{\sim}{\rightarrow} \underline{k}^{\times}\left(H_{\bullet}\right) \hookleftarrow \underline{k}^{\times}\left(G_{\bullet}\right)
$$

-where the first arrow is an isomorphism, and the second arrow is injective.

(iii) The vertical open homomorphisms $\mathcal{O}^{\times}\left(G_{\circ}\right) \rightarrow \mathcal{O}^{\times}\left(H_{\bullet}\right) \hookleftarrow \mathcal{O}^{\times}\left(G_{\bullet}\right)$ in the diagram of (i) fit into a commutative diagram of topological modules

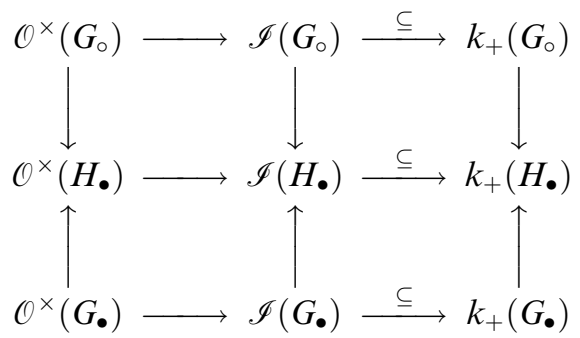


- where the horizontal arrows are the natural homomorphisms, the upper vertical arrows are surjective, and the lower vertical arrows are injective.

Proof. These assertions follow immediately from Proposition 3.4, (i), (iii), (iv).

REMARK 3.5.1. It follows immediately from Proposition 3.4, (iv), that the vertical surjection $k^{\times}\left(G_{\circ}\right) \rightarrow k^{\times}\left(H_{\bullet}\right)$ in the diagram of Proposition 3.5, (i), fits into a commutative diagram of modules

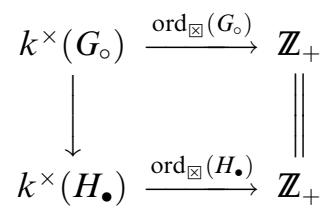

[cf. Definition 2.2].

TheOREM 3.6. For each $\square \in\{0, \bullet\}$, let $G_{\square}$ be a profinite group of MLFtype. Let

$$
\alpha: G_{\circ} \rightarrow G_{\bullet}
$$

be an open homomorphism. Suppose that $d\left(G_{\circ}\right) \leq d\left(G_{\bullet}\right)$ [which is the case if, for instance, $d\left(G_{\circ}\right)=1$ ]. Then $\alpha$ is an isomorphism.

Proof. Since $d\left(G_{\circ}\right) \leq d\left(G_{\bullet}\right)$, by applying Proposition 3.4, (iii), to the natural surjection $G_{\circ} \rightarrow \alpha\left(G_{\circ}\right)$ and the natural inclusion $\alpha\left(G_{\circ}\right) \hookrightarrow G_{\bullet}$. [note that $\alpha\left(G_{\circ}\right)$ is of MLF-type - cf. the discussion following [3], Proposition 3.3], we obtain that $d\left(\alpha\left(G_{\circ}\right)\right)=d\left(G_{\bullet}\right)$. On the other hand, it follows from [3], Proposition 3.6, that this equality implies the equality $\alpha\left(G_{\circ}\right)=G_{\bullet}$, i.e., that $\alpha$ is surjective.

Now assume that $\alpha$ is not injective, i.e., that $J \stackrel{\text { def }}{=} \operatorname{Ker}(\alpha)$ is nontrivial. Let us first observe that since $J$ is contained in $P\left(G_{\circ}\right)$ [cf. Proposition 3.4, (ii)], the profinite group $J$ is pro-p $\left(G_{\circ}\right)$, which thus implies that $J$ does not satisfy condition (3) of Lemma 3.3, (i). Thus, it follows from Lemma 3.3, (iv), that there exists an open subgroup $H_{\circ} \subseteq G_{\circ}$ of $G_{\circ}$ such that $J \subseteq H_{\circ}$ [i.e., $\left.H_{\circ}=\alpha^{-1}\left(\alpha\left(H_{\circ}\right)\right)\right]$, and, moreover, the image of $J$ in $\left(H_{\circ}^{\left(p\left(G_{\circ}\right)\right)}\right)^{\text {ab/tor }}$ is nontrivial. In particular, since $d\left(H_{\circ}\right)=d\left(G_{\circ}\right) \cdot\left[G_{\circ}: H_{\circ}\right] \leq d\left(G_{\bullet}\right) \cdot\left[G_{\circ}: H_{\circ}\right]=$ $d\left(G_{\bullet}\right) \cdot\left[G_{\bullet}: \alpha\left(H_{\circ}\right)\right]=d\left(\alpha\left(H_{\circ}\right)\right)$ [cf. [3], Proposition 3.6], we may assume without loss of generality, by replacing $\left(G_{\circ}, G_{\bullet}\right)$ by $\left(H_{\circ}, \alpha\left(H_{\circ}\right)\right)$, that the image of $J$ in $\left(G_{\circ}^{\left(p\left(G_{\circ}\right)\right)}\right)^{\text {ab/tor }}$ is nontrivial. On the other hand, this implies that the surjection $\left(G_{\circ}^{\left(p\left(G_{\circ}\right)\right)}\right)^{\text {ab/tor }} \rightarrow\left(G_{\bullet}^{\left(p\left(G_{\circ}\right)\right)}\right)^{\text {ab/tor }}=\left(G_{\bullet}^{\left(p\left(G_{\bullet}\right)\right)}\right)^{\text {ab/tor }}$ [cf. Proposition 3.4, (iii)] induced by $\alpha$ is not injective. Thus, it follows immediately from Lemma 
3.1, (i), that $d\left(G_{\circ}\right)>d\left(G_{\bullet}\right)$ - in contradiction to our assumption that $d\left(G_{\circ}\right) \leq$ $d\left(G_{\bullet}\right)$. This completes the proof of Theorem 3.6.

Corollary 3.7. For each $\square \in\{\circ, \bullet\}$, let $G_{\square}$ be a profinite group of MLFtype. Let

$$
\alpha: G_{\circ} \rightarrow G_{\bullet}
$$

be an open homomorphism. Suppose that $e\left(G_{\circ}\right) \leq e\left(G_{\bullet}\right)$ [which is the case if, for instance, $e\left(G_{\circ}\right)=1$ ]. Then $\alpha$ is injective.

Proof. Since $e\left(G_{\circ}\right) \leq e\left(G_{\bullet}\right)$, by applying Proposition 3.4, (iii), to the natural surjection $G_{\circ} \rightarrow \alpha\left(G_{\circ}\right)$ and the natural inclusion $\alpha\left(G_{\circ}\right) \hookrightarrow G_{\bullet}$. [note that $\alpha\left(G_{\circ}\right)$ is of MLF-type-cf. the discussion following [3], Proposition 3.3], we obtain that $e\left(G_{\circ}\right)=e\left(\alpha\left(G_{\circ}\right)\right)$. Thus, to verify Corollary 3.7, we may assume without loss of generality, by replacing $G_{\bullet}$ by $\alpha\left(G_{\circ}\right)$, that $\alpha$ is surjective, and that $e\left(G_{\circ}\right)=e\left(G_{\bullet}\right)$. Then since $\alpha$ is surjective, and $e\left(G_{\circ}\right)=e\left(G_{\bullet}\right)$, it follows immediately from Proposition 3.4, (iii), that $d\left(G_{\circ}\right)=d\left(G_{\bullet}\right)$. Thus, it follows from Theorem 3.6 that $\alpha$ is an isomorphism, as desired. This completes the proof of Corollary 3.7.

Corollary 3.8. For each $\square \in\{\circ, \bullet\}$, let $k_{\square}$ be an MLF and $\bar{k}_{\square}$ an algebraic closure of $k_{\square}$; write $G_{\square} \stackrel{\text { def }}{=} \operatorname{Gal}\left(\bar{k}_{\square} / k_{\square}\right)$. Suppose that $e_{k_{\circ}}=1$. Then the following three conditions are equivalent:

(1) The field $k_{\circ}$ is isomorphic to the field $k_{\bullet}$.

(2) There exists a surjection $G_{\circ} \rightarrow G_{\text {o }}$.

(3) The group $G_{\circ}$ is isomorphic to the group $G_{\bullet}$.

Proof. The implication $(1) \Rightarrow(2)$ is immediate. The implication $(2) \Rightarrow(3)$ follows - in light of [3], Proposition 3.6-from Corollary 3.7. Finally, since [we have assumed that] $e_{k_{\circ}}=1$, the implication $(3) \Rightarrow(1)$ follows immediately from [3], Proposition 3.6 [cf. also [3], Lemma 1.5, (i)]. This completes the proof of Corollary 3.8 .

REMARK 3.8.1. Suppose that we are in the situation of Corollary 3.8, that $p_{k_{\circ}} \neq 2$, and that the conditions (1), (2), and (3) of Corollary 3.8 hold. Then since $e_{k_{\circ}}=1$, one verifies easily that the MLF $k_{\circ}$ is absolutely abelian [cf. Definition 4.2, (ii), below], hence also [cf. Theorem 6.3, (i), below] absolutely characteristic [cf. Definition 5.7 below]. Thus, it follows from Theorem 7.2, (i), below that there exists an outer automorphism of $G_{\circ}$ that does not arise from any field automorphism of $k_{\circ}$. In particular, there exists an outer isomorphism $G_{\circ} \stackrel{\sim}{\rightarrow} G_{\bullet}$ [cf. condition (3) of Corollary 3.8] that does not arise from any field isomorphism $k_{\bullet} \stackrel{\sim}{\rightarrow} k_{\circ}$ [cf. condition (1) of Corollary 3.8]. 


\section{Reconstruction algorithms related to absolutely abelian MLF's}

In the present $\S 4$, we maintain the notational conventions introduced at the beginnings of $\S 1$ and $\S 2$. In the present $\S 4$, we discuss some group-theoretic reconstruction algorithms [cf. [8], Remark 1.9.8] related to absolutely abelian [cf. Definition 4.2, (ii), below] MLF's. We establish, for instance, a grouptheoretic reconstruction algorithm for constructing, from a group of MLF-type, a homomorphism which "corresponds" to the Norm map $\mathrm{Nm}_{k / k^{(d=1)}}: k^{\times} \rightarrow$ $\left(k^{(d=1)}\right)^{\times}$with respect to the finite extension $k / k^{(d=1)}$ [cf. Definition 4.7, (iii); Proposition 4.9, (i), below], which leads us to the notion of MLF-Galois label [cf. Definition 4.10, Theorem 4.11 below]. Finally, as a consequence of the group-theoretic reconstruction algorithms, we also obtain a refinement of the main theorem of [6] [cf. Corollary 4.14, (i); Remark 4.14.1 below].

LEMMA 4.1. The following hold:

(i) The natural homomorphisms

$$
\mathbb{Z}_{p_{k}} \rightarrow \operatorname{End}_{\mathbb{Z}_{p_{k}}}\left(\Lambda(\bar{k})^{\left(p_{k}\right)}\right), \quad \mathbb{Q}_{p_{k}} \rightarrow \operatorname{End}_{\mathbb{Q}_{p_{k}}}\left(\Lambda(\bar{k})^{\left(p_{k}\right)} \otimes_{\mathbb{Z}_{p_{k}}} \mathbb{Q}_{p_{k}}\right)
$$

are isomorphisms of topological algebras. Moreover, these isomorphisms restrict to isomorphisms of topological groups

$$
\mathbb{Z}_{p_{k}}^{\times} \stackrel{\sim}{\rightarrow} \operatorname{Aut}_{\mathbb{Z}_{p_{k}}}\left(\Lambda(\bar{k})^{\left(p_{k}\right)}\right), \quad \mathbb{Q}_{p_{k}}^{\times} \stackrel{\sim}{\rightarrow} \operatorname{Aut}_{\mathbb{Q}_{p_{k}}}\left(\Lambda(\bar{k})^{\left(p_{k}\right)} \otimes_{\mathbb{Z}_{p_{k}}} \mathbb{Q}_{p_{k}}\right),
$$

respectively.

We shall write

$$
\chi_{p_{k} \text {-cyc }}: G_{k} \rightarrow \mathbb{Z}_{p_{k}}^{\times}
$$

for the composite of the natural action $G_{k} \rightarrow \operatorname{Aut}_{\mathbb{Z}_{p_{k}}}\left(\Lambda(\bar{k})^{\left(p_{k}\right)}\right)$ and the above isomorphism $\operatorname{Aut}_{\mathbb{Z}_{p_{k}}}\left(\Lambda(\bar{k})^{\left(p_{k}\right)}\right) \stackrel{\sim}{\leftarrow} \mathbb{Z}_{p_{k}}^{\times}$, i.e., the $p_{k}$-adic cyclotomic character.

(ii) Let $\overline{\mathbb{Q}}_{p_{k}}$ be an algebraic closure of $\mathbb{Q}_{p_{k}}$. Then the homomorphism $G_{k}^{\mathrm{ab}} \rightarrow \mathbb{Z}_{p_{k}}^{\times}$determined by the $p_{k}$-adic cyclotomic character $\chi_{p_{k} \text {-cyc }}: G_{k} \rightarrow \mathbb{Z}_{p_{k}}^{\times}[c f$. (i)] coincides with the composite

$$
\begin{aligned}
& G_{k}^{\mathrm{ab}} \rightarrow \operatorname{Gal}\left(\bar{k} / k^{(d=1)}\right)^{\mathrm{ab}} \stackrel{\sim}{\rightarrow} \operatorname{Gal}\left(\overline{\mathbb{Q}}_{p_{k}} / \mathbb{Q}_{p_{k}}\right)^{\mathrm{ab}}
\end{aligned}
$$

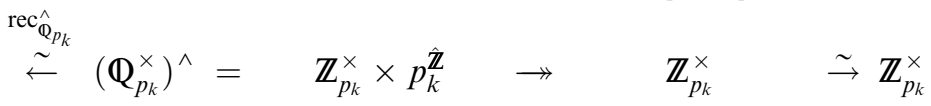

-where the first arrow " $\rightarrow$ " is the homomorphism induced by the natural inclusion $G_{k} \hookrightarrow \operatorname{Gal}\left(\bar{k} / k^{(d=1)}\right)$; the second arrow “ $\stackrel{\sim}{\rightarrow}$ " is the isomorphism induced by an isomorphism $\overline{\mathbb{Q}}_{p_{k}} \stackrel{\sim}{\rightarrow} \bar{k}$ of fields [that necessarily restricts to an isomorphism $\mathbb{Q}_{p_{k}} \stackrel{\sim}{\rightarrow} k^{(d=1)}$ of fields]; the third arrow $\operatorname{rec}_{\mathbb{Q}_{p_{k}}}$ is the isomorphism in the final display of [3], Lemma 1.7, (1) [in the case where we take the " $k$ " of [3], Lemma 1.7 , to be $\left.\mathbb{Q}_{p_{k}}\right]$; the fourth arrow " $\rightarrow$ " is the first projection; the fifth arrow " $\rightarrow$ ", is the isomorphism given by " $a \mapsto a^{-1}$ ". 
(iii) The composite of the Norm map $\mathrm{Nm}_{k / k^{(d=1)}}: k^{\times} \rightarrow\left(k^{(d=1)}\right)^{\times}$with respect to the finite extension $k / k^{(d=1)}$ and the isomorphism $\left(k^{(d=1)}\right)^{\times} \stackrel{\sim}{\rightarrow} \mathbb{Q}_{p_{k}}^{\times}$ induced by the [uniquely determined] isomorphism $k^{(d=1)} \stackrel{\sim}{\rightarrow} \mathbb{Q}_{p_{k}}$ of fields coincides with the homomorphism $k^{\times} \rightarrow \mathbb{Q}_{p_{k}}^{\times}$given by

$$
k^{\times} \ni a \mapsto \chi_{p_{k} \text {-cyc }}\left(\operatorname{rec}_{k}(a)\right)^{-1} \cdot p_{k}^{f_{k} \cdot \operatorname{ord}_{k}(a)} \in \mathbb{Q}_{p_{k}}^{\times}
$$

[cf. [3], Lemma 1.7].

Proof. Assertion (i) follows from the [easily verified] fact that the $\mathbb{Z}_{p_{k}}$-module $\Lambda(\bar{k})^{\left(p_{k}\right)}$ is free of rank one. Assertion (ii) follows immediately from the well-known [cf., e.g., [15], Chapter III, § A.4, Corollary] fact that the $p_{k}$-adic cyclotomic character in the case where we take the " $k$ " to be the MLF $\mathbb{Q}_{p_{k}}$ coincides with the "Lubin-Tate character $\chi_{\sigma, \pi}^{\mathrm{LT}}$ " [cf. the notational convention introduced in [1], Definition 1.2, (ii)] in the case where we take the " $E$ " (respectively, " $\sigma$ "; " $\pi$ ") of [1], Definition 1.2, (ii), to be $\mathbb{Q}_{p_{k}}$ (respectively, the identity automorphism of $\mathbb{Q}_{p_{k}} ; p_{k} \in \mathcal{O}_{\mathbb{Q}_{p_{k}}}=\mathbb{Z}_{p_{k}}$ ). Assertion (iii) follows immediately from assertion (ii) and [3], Lemma 1.7, (1), (2). This completes the proof of Lemma 4.1 .

DEFINITION 4.2.

(i) We shall say that the MLF $k$ is absolutely Galois if $k$ is Galois over $k^{(d=1)}$.

(ii) We shall say that the MLF $k$ is absolutely abelian if $k$ is absolutely Galois, and, moreover, the Galois group $\operatorname{Gal}\left(k / k^{(d=1)}\right)$ is abelian.

(iii) We shall write $k^{(\mathrm{ab})} \subseteq k$ for the [uniquely determined] maximal absolutely abelian MLF contained in $k$.

(iv) We shall write

$$
d_{k}^{(\mathrm{ab}) \stackrel{\text { def }}{=}} d_{k^{(\mathrm{ab})}}, \quad e_{k}^{(\mathrm{ab})} \stackrel{\text { def }}{=} e_{k^{(\mathrm{ab})}}
$$

for the " $d_{k}$ ", " $e_{k}$ " in the case where we take the " $k$ " to be $k^{(\mathrm{ab})}$ of (iii), respectively.

LEMMA 4.3. Let $K$ be an intermediate field of the finite extension $k / k^{(\mathrm{ab})}$. Then the following hold:

(i) It holds that $K^{(\mathrm{ab})}=k^{(\mathrm{ab})}$.

(ii) There is no nontrivial intermediate field of the finite extension $k / k^{(\mathrm{ab})}$, hence also of $K / k^{(\mathrm{ab})}$, that is unramified over $k^{(\mathrm{ab})}$.

Proof. Assertion (i) follows from the definition of " $(-)^{(a b)}$ ", Assertion (ii) follows immediately from the [easily verified] fact that every intermediate field of $k / k^{(\mathrm{ab})}$ unramified over $k^{(\mathrm{ab})}$ is absolutely abelian [cf. [3], Lemma 1.5, (i)]. This completes the proof of Lemma 4.3. 
LEMMA 4.4. The following hold:

(i) It holds that

$$
d_{k}^{(\mathrm{ab})}=e_{k}^{(\mathrm{ab})} \cdot f_{k}, \quad d_{k}^{(\mathrm{ab})}=\left[\left(k^{(d=1)}\right)^{\times}: \mathrm{Nm}_{k / k^{(d=1)}}\left(k^{\times}\right)\right] .
$$

(ii) The following three conditions are equivalent:

(1) The $M L F k$ is absolutely abelian.

(2) It holds that $d_{k}=d_{k}^{(\mathrm{ab})}$.

(3) It holds that $e_{k}=e_{k}^{(\mathrm{ab})}$.

Proof. First, we verify assertion (i). The equality $d_{k}^{(\mathrm{ab})}=e_{k}^{(\mathrm{ab})} \cdot f_{k}$ follows from Lemma 4.3, (ii), together with [3], Lemma 1.2, (iii). The equality $d_{k}^{(\mathrm{ab})}=\left[\left(k^{(d=1)}\right)^{\times}: \mathrm{Nm}_{k / k^{(d=1)}}\left(k^{\times}\right)\right]$follows immediately from [3], Lemma 1.7, (1), (2). This completes the proof of assertion (i). Assertion (ii) follows immediately from assertion (i), together with [3], Lemma 1.2, (iii). This completes the proof of Lemma 4.4 .

Recall the group of MLF-type

$$
G
$$

introduced at the beginning of $\S 2$.

DEFINITION 4.5.

(i) We shall write

$$
\Lambda(G)^{(p(G))}
$$

for the maximal pro- $p(G)$ quotient of the cyclotome $\Lambda(G)$ associated to $G$. Note that since $\Lambda(G)^{(p(G))}$ has a natural structure of free $\mathbb{Z}_{p(G)}$-module of rank one [cf. [3], Proposition 4.2, (iv)], the perfection

$$
\left(\Lambda(G)^{(p(G))}\right)^{\mathrm{pf}}
$$

of $\Lambda(G)^{(p(G))}$ has a natural structure of $\mathbb{Q}_{p(G)}$-vector space of dimension one.

(ii) We shall write

$$
\mathbb{Z}_{p}(G) \stackrel{\text { def }}{=} \operatorname{End}\left(\Lambda(G)^{(p(G))}\right)
$$

for the topological algebra of endomorphisms of the topological module $\Lambda(G)^{(p(G))}$.

(iii) We shall write

$$
\mathbb{Q}_{p}(G) \stackrel{\text { def }}{=} \operatorname{End}\left(\left(\Lambda(G)^{(p(G))}\right)^{\mathrm{pf}}\right)
$$

for the algebra of endomorphisms of the perfection $\left(\Lambda(G)^{(p(G))}\right)^{\mathrm{pf}}$. Thus, we have a natural inclusion

$$
\mathbb{Z}_{p}(G) \hookrightarrow \mathbb{Q}_{p}(G)
$$


By considering the topology induced by the topology of $\mathbb{Z}_{p}(G)$ [cf. (ii)], we regard $\mathbb{Q}_{p}(G)$ as a topological algebra.

LEMMA 4.6. The following hold:

(i) The natural homomorphism

$$
\mathbb{Z}_{p(G)} \rightarrow \mathbb{Z}_{p}(G)
$$

[i.e., obtained by the natural $\mathbb{Z}_{p(G)}$-module structure of $\Lambda(G)^{p(G)}$ ] is an isomorphism of topological algebra. Moreover, this isomorphism determines an isomorphism of topological algebra

$$
\mathbb{Q}_{p(G)} \stackrel{\sim}{\rightarrow} \mathbb{Q}_{p}(G) .
$$

(ii) We have natural identifications

$$
\mathbb{Z}_{p}(G)^{\times}=\operatorname{Aut}\left(\Lambda(G)^{p(G)}\right) \subseteq \mathbb{Q}_{p}(G)^{\times}=\operatorname{Aut}\left(\left(\Lambda(G)^{p(G)}\right)^{\mathrm{pf}}\right)
$$

[cf. (i)].

Proof. These assertions follow immediately - in light of [3], Proposition 3.6; [3], Proposition 4.2, (iv)-from Lemma 4.1, (i).

DEFINITION 4.7.

(i) We shall write

$$
\chi_{p-\mathrm{cyc}}(G): G \rightarrow \mathbb{Z}_{p}(G)^{\times}
$$

for the natural action of $G$ on $\Lambda(G)^{p(G)}$ [cf. Lemma 4.6, (ii)].

(ii) We shall write

$$
p \in(G) \in \mathbb{Q}_{p}(G)^{\times}
$$

for the automorphism of the module $\left(\Lambda(G)^{p(G)}\right)^{\text {pf }}$ given by multiplication by $p(G)$ [cf. Lemma 4.6, (ii)].

(iii) We shall write

$$
\mathrm{Nm}_{\mathrm{abs}}(G): k^{\times}(G) \rightarrow \mathbb{Q}_{p}(G)^{\times}
$$

for the homomorphism of topological modules defined by

$$
k^{\times}(G) \ni a \mapsto \chi_{p \text {-cyc }}(G)(\operatorname{rec}(G)(a))^{-1} \cdot p \in(G)^{f(G) \cdot \operatorname{ord}_{凶}(G)(a)} \in \mathbb{Q}_{p}(G)^{\times}
$$

[cf. Definition 2.2].

(iv) We shall write

$$
d^{(\mathrm{ab})}(G) \stackrel{\text { def }}{=}\left[\mathbb{Q}_{p}(G)^{\times}: \operatorname{Im}\left(\mathrm{Nm}_{\mathrm{abs}}(G)\right)\right], \quad e^{(\mathrm{ab})}(G) \stackrel{\text { def }}{=} d^{(\mathrm{ab})}(G) / f(G) .
$$


DeFINITION 4.8. We shall say that $G$ is of AAMLF-type if $d(G)=$ $d^{(\mathrm{ab})}(G)$. [Here, "AAMLF" is to be understood as an abbreviation for "absolutely abelian mixed-characteristic local field"-cf. Proposition 4.9, (iii), below.]

Proposition 4.9. The following hold:

(i) Write $\mathrm{Nm}_{k / k^{(d=1)}}: k^{\times} \rightarrow\left(k^{(d=1)}\right)^{\times}$for the Norm map with respect to the finite extension $k / k^{(d=1)}$. Then the vertical isomorphism $k^{\times} \stackrel{\sim}{\rightarrow} k^{\times}\left(G_{k}\right)$ in the diagram of [3], Proposition 3.11, (i), fits into a commutative diagram of topological modules

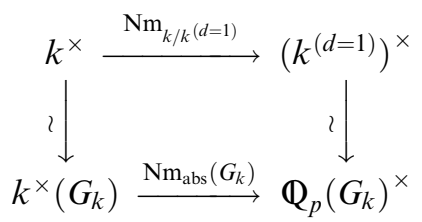

-where the right-hand vertical arrow is the composite of the isomorphism $\left(k^{(d=1)}\right)^{\times} \stackrel{\sim}{\rightarrow} \mathbb{Q}_{p\left(G_{k}\right)}^{\times}$induced by the [uniquely determined] isomorphism $k^{(d=1)} \stackrel{\sim}{\rightarrow}$ $\mathbb{Q}_{p\left(G_{k}\right)}$ of fields and the isomorphism $\mathbb{Q}_{p_{k}}^{\times}=\mathbb{Q}_{p\left(G_{k}\right)}^{\times} \stackrel{\sim}{\rightarrow} \mathbb{Q}_{p}\left(G_{k}\right)^{\times}[c f$. [3], Proposition 3.6] determined by the isomorphism of Lemma 4.6, (i).

(ii) It holds that

$$
d_{k}^{(\mathrm{ab})}=d^{(\mathrm{ab})}\left(G_{k}\right), \quad e_{k}^{(\mathrm{ab})}=e^{(\mathrm{ab})}\left(G_{k}\right) .
$$

(iii) It holds that the MLF $k$ is absolutely abelian if and only if the group $G_{k}$ is of AAMLF-type.

Proof. Assertion (i) follows - in light of Proposition 2.3; [3], Proposition 3.6; [3], Proposition 3.11, (i); [3], Proposition 4.2, (iv)—from Lemma 4.1, (iii). Assertion (ii) follows immediately from Lemma 4.4, (i), together with assertion (i) and [3], Proposition 3.6. Assertion (iii) follows from Lemma 4.4, (ii), together with assertion (ii) and [3], Proposition 3.6. This completes the proof of Proposition 4.9.

REMARK 4.9.1. Let $H \subseteq G$ be an open subgroup of $G$. Then one verifies immediately from Proposition 4.9, (i), together with [3], Lemma 1.7, (2), that the diagram of topological modules

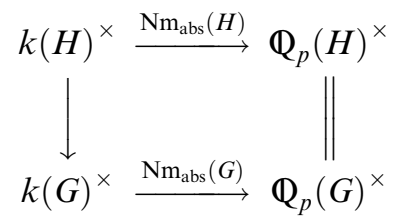


-where the left-hand vertical arrow is the homomorphism induced by the homomorphism $H^{\mathrm{ab}} \rightarrow G^{\mathrm{ab}}$ determined by the inclusion $H \hookrightarrow G$, and the righthand vertical arrow is the composite of the isomorphisms $\mathbb{Q}_{p(G)}^{\times} \stackrel{\sim}{\rightarrow} \mathbb{Q}_{p}(G)^{\times}$, $\mathbb{Q}_{p(H)}^{\times} \stackrel{\sim}{\rightarrow} \mathbb{Q}_{p}(H)^{\times}$determined by the isomorphism of Lemma 4.6, (i) [cf. also [3], Proposition 3.6]-commutes.

REMARK 4.9.2. Suppose that $G$ is of AAMLF-type. Then a profinite group isomorphic to $G$ may be constructed as follows: Let $\tilde{G}$ be a group of MLF-type such that $(p(\tilde{\boldsymbol{G}}), d(\tilde{\boldsymbol{G}}))=(p(G), 1)$. [Note that one verifies easily from [3], Proposition 3.6, that this condition $(p(\tilde{G}), d(\tilde{G}))=(p(G), 1)$ completely determines the isomorphism class of the group $\tilde{G}$.] Write $J \subseteq \tilde{G}^{\text {ab }}$ for the closure, i.e., in $\tilde{G}^{\mathrm{ab}}$, of the inverse image $\left(\subseteq k^{\times}(\tilde{G}) \subseteq \tilde{G}^{\mathrm{ab}}\right)$ of $\operatorname{Im}\left(\operatorname{Nm}_{\mathrm{abs}}(G)\right)$ $\subseteq \mathbb{Q}_{p}(G)^{\times}$by $\operatorname{Nm}_{\text {abs }}(\tilde{\boldsymbol{G}}): k^{\times}(\tilde{\boldsymbol{G}}) \rightarrow \mathbb{Q}_{p}(\tilde{\boldsymbol{G}})^{\times}$-relative to the composite of the isomorphisms $\mathbb{Q}_{p(G)}^{\times} \stackrel{\sim}{\rightarrow} \mathbb{Q}_{p}(G)^{\times}, \mathbb{Q}_{p(\tilde{\boldsymbol{G}})}^{\times} \stackrel{\sim}{\rightarrow} \mathbb{Q}_{p}(\tilde{\boldsymbol{G}})^{\times}$determined by the isomorphism of Lemma 4.6, (i). Then it follows immediately from Remark 4.9.1 that $G$ is isomorphic, as an abstract profinite group, to the inverse image of $J \subseteq \tilde{G}^{\text {ab }}$ in $\tilde{G}$.

Definition 4.10. We shall refer to the collection of data

$$
\left(p(G), d(G), \operatorname{Im}\left(\mathrm{Nm}_{\mathrm{abs}}(G)\right) \subseteq \mathbb{Q}_{p}(G)^{\times} \check{\leftarrow} \mathbb{Q}_{p(G)}^{\times}\right)
$$

[cf. Lemma 4.6, (i)] consisting of the prime number $p(G)$, the positive integer $d(G)$, and the open subgroup $\operatorname{Im}\left(\mathrm{Nm}_{\mathrm{abs}}(G)\right) \subseteq \mathbb{Q}_{p(G)}^{\times}$of $\mathbb{Q}_{p(G)}^{\times}$as the MLFGalois label of $G$.

THEOREM 4.11. For each $\square \in\{\circ, \bullet\}$, let $G_{\square}$ be a group of MLF-type. Suppose that one of the following two conditions is satisfied:

(1) It holds that $\left\{\left(p\left(G_{\circ}\right), a\left(G_{\circ}\right)\right),\left(p\left(G_{\bullet}\right), a\left(G_{\bullet}\right)\right)\right\} \nsubseteq\{(2,1)\}[c f$. Definition 2.4, (ii)].

(2) Either $G_{\circ}$ or $G_{\bullet}$ is of AAMLF-type.

Then it holds that the group $G_{\circ}$ is isomorphic to the group $G_{\bullet}$ if and only if the $M L F$-Galois label of $G_{\circ}$ coincides with the MLF-Galois label of $G_{\bullet}$.

Proof. The necessity is immediate. Next, we verify the sufficiency in the case where condition (1) is satisfied. Suppose that condition (1) is satisfied, and that the MLF-Galois label of $G_{\circ}$ coincides with the MLF-Galois label of $G_{\bullet}$. Then since $\operatorname{Im}\left(\mathrm{Nm}_{\mathrm{abs}}\left(G_{\circ}\right)\right)=\operatorname{Im}\left(\mathrm{Nm}_{\mathrm{abs}}\left(G_{\bullet}\right)\right)$, one verifies immediately from Proposition 2.5, (i); Proposition 4.9, (i); [3], Lemma 1.7, (1), (2); [3], Proposition 3.6, that $(2,1) \notin\left\{\left(p\left(G_{\circ}\right), a\left(G_{\circ}\right)\right),\left(p\left(G_{\bullet}\right), a\left(G_{\bullet}\right)\right)\right\}$. Thus, since the MLF-Galois label of $G_{\circ}$ coincides with the MLF-Galois label of $G_{\bullet}$, it follows immediately - in light of Proposition 2.5, (i); Proposition 4.9, (i); [3], Proposition 3.6-from the main theorems of [4] and [13], together 
with [3], Lemma 1.7, (1), (2), that $G_{\circ}$ is isomorphic to $G_{\bullet}$, as desired. This completes the proof of the sufficiency in the case where condition (1) is satisfied.

Finally, we verify the sufficiency in the case where condition (2) is satisfied. Suppose that $G_{\circ}$ is of AAMLF-type, and that the MLF-Galois label of $G_{\circ}$ coincides with the MLF-Galois label of $G_{\bullet}$. Then since $\operatorname{Im}\left(\mathrm{Nm}_{\mathrm{abs}}\left(G_{\circ}\right)\right)=$ $\operatorname{Im}\left(\mathrm{Nm}_{\mathrm{abs}}\left(G_{\bullet}\right)\right)$, we obtain that $d^{(\mathrm{ab})}\left(G_{\circ}\right)=d^{(\mathrm{ab})}\left(G_{\bullet}\right)$. In particular, since $G_{\circ}$ is of $A A M L F$-type, the equality $d\left(G_{\circ}\right)=d\left(G_{\bullet}\right)$ implies that $G_{\bullet}$ is of $A A M L F$ type. Thus, since the MLF-Galois label of $G_{\circ}$ coincides with the MLF-Galois label of $G_{\bullet}$, it follows immediately from Remark 4.9.2 that $G_{\circ}$ is isomorphic to $G_{\bullet}$, as desired. This completes the proof of the sufficiency in the case where condition (2) is satisfied, hence also of Theorem 4.11 .

REMARK 4.11.1.

(i) Let us recall that the main theorem of [1] asserts that, roughly speaking, the Hodge-Tate-ness of $p_{k}$-adic representations of the group $G_{k}$ of MLFtype is closely related to the ring structures of the fields $k \subseteq \bar{k}$.

(ii) Let us also recall that, as discussed in [3], Proposition 4.2, (iv), the $p_{k}$-adic cyclotomic character may be "reconstructed" from just the group structure of the group $G_{k}$ of MLF-type.

Next, let us recall that Theorem 4.11 asserts that-under a mild assumption on " $\left(p_{k}, a_{k}\right)$ " - the isomorphism class of the group $G_{k}$ is completely determined by the MLF-Galois label of $G_{k}$. Now observe that the main component of the notion of MLF-Galois label is the third component, i.e., the image of the Norm map to $\left(k^{(d=1)}\right)^{\times}$. Moreover, recall that, as discussed in Lemma 4.1, (iii), roughly speaking, the Norm map to $\left(k^{(d=1)}\right)^{\times}$may be essentially described by the $p_{k}$-adic cyclotomic character.

Thus, one may conclude that, roughly speaking, the $p_{k}$-adic cyclotomic character is closely related to the group structure of the group $G_{k}$ of MLFtype.

(iii) It follows from the observations of (i), (ii), together with [3], Remark 4.3.3, that, in summary,

Hodge-Tate representations is closely related to arithmetic holomorphic structures [i.e., roughly speaking, ring structures - cf. [9], §2.7, (vii)] of MLF's,

and, moreover,

the cyclotomic character [that is one of Hodge-Tate representations] is closely related to mono-analytic structures [i.e., roughly speaking, structures that arise from dismantling the complicated intertwining inherent in ring structures-cf. [9], §2.7, (vii)] of MLF's. 
Hodge-Tate representations $\Leftrightarrow$ arithmetic holomorphic structures of MLF's

$$
\begin{array}{cc}
\Rightarrow: & \text { the main theorem of }[1] \\
\Leftarrow: & \text { immediate }
\end{array}
$$

cyclotomic characters $\quad \Leftrightarrow \quad$ mono-analytic structures of MLF's

$\Rightarrow$ : $\quad$ Lemma 4.1, (iii), and Theorem 4.11

$\Leftarrow: \quad$ [3], Proposition 4.2, (iv)

Definition 4.12. For each $\square \in\{0, \bullet\}$, let $G_{\square}$ be a group of MLF-type. Let $\alpha: G_{\circ} \rightarrow G_{\bullet}$ be a homomorphism. Then we shall say that $\alpha$ is cyclotomically compatible if $p\left(G_{\circ}\right)=p\left(G_{\bullet}\right)$, and, moreover, the diagram

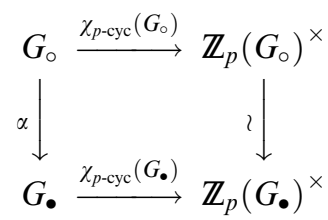

-where the right-hand vertical arrow is the composite of the isomorphisms $\mathbb{Z}_{p\left(G_{\circ}\right)}^{\times} \stackrel{\sim}{\rightarrow} \mathbb{Z}_{p}\left(G_{\circ}\right)^{\times}, \mathbb{Z}_{p\left(G_{\bullet}\right)}^{\times} \stackrel{\sim}{\rightarrow} \mathbb{Z}_{p}\left(G_{\bullet}\right)^{\times}$determined by the isomorphism of Lemma 4.6, (i)-commutes.

REMARK 4.12.1. Let us recall that it follows immediately from the various definitions involved that every open injection between profinite groups of MLFtype induces a natural isomorphism between the cyclotomes " $\Lambda(-)$ " [cf. [3], Definition 4.1, (i), (ii), (iii)]. In particular, every open injection between profinite groups of MLF-type is cyclotomically compatible.

REMARK 4.12.2. Let $l$ be a prime number such that $l \neq p(G)$. Then, in the situation of Definition 4.7, (i), by considering the natural action on the maximal pro-l quotient of the cyclotome $\Lambda(G)$ [i.e., as opposed to the natural action on $\Lambda(G)^{p(G)}$ discussed in Definition 4.7, (i)], one may define the notion of "l-adic cyclotomic character" of $G$ [i.e., as opposed to the " $p(G)$-adic cyclotomic character" $\chi_{p \text {-cyc }}(G)$ defined in Definition 4.7, (i)].

Now let us observe that it follows immediately - in light of [3], Proposition 3.6; [3], Proposition 4.2, (iv)-from Proposition 3.4, (iii), (iv), and [3], Lemma 1.5 , (i), (ii), (iii), that every open homomorphism between profinite groups of MLF-type is compatible with the respective "l-adic cyclotomic characters", i.e., that a similar diagram to the diagram of Definition 4.12 commutes.

THEOREM 4.13. For each $\square \in\{\circ, \bullet\}$, let $G_{\square}$ be a profinite group of MLFtype. Let

$$
\alpha: G_{\circ} \rightarrow G_{\bullet}
$$

be an open homomorphism. Then the following hold: 
(i) If $\alpha$ is cyclotomically compatible and surjective, then the surjection $k^{\times}\left(G_{\circ}\right) \rightarrow k^{\times}\left(G_{\bullet}\right)$ induced by $\alpha[c f$. Proposition 3.5, (i)] fits into a commutative diagram of topological modules

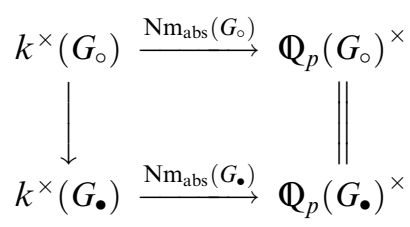

-where the right-hand vertical arrow is the composite of the isomorphisms $\mathbb{Q}_{p\left(G_{\circ}\right)}^{\times} \stackrel{\sim}{\rightarrow} \mathbb{Q}_{p}\left(G_{\circ}\right)^{\times}, \mathbb{Q}_{p\left(G_{\bullet}\right)}^{\times} \stackrel{\sim}{\rightarrow} \mathbb{Q}_{p}\left(G_{\bullet}\right)^{\times}$determined by the isomorphism of Lemma 4.6, (i) [cf. also Proposition 3.4, (iii)]. Moreover, it holds that

$$
d^{(\mathrm{ab})}\left(G_{\circ}\right)=d^{(\mathrm{ab})}\left(G_{\bullet}\right), \quad e^{(\mathrm{ab})}\left(G_{\circ}\right)=e^{(\mathrm{ab})}\left(G_{\bullet}\right) .
$$

(ii) If $G_{\circ}$ is of AAMLF-type, then the following two conditions are equivalent:

(1) The homomorphism $\alpha$ is injective.

(2) The homomorphism $\alpha$ is cyclotomically compatible.

(iii) In the situation of (ii), if, moreover, (1) and (2) of (ii) are satisfied, then the group $G$. is of AAMLF-type.

Proof. First, we verify assertion (i). The first assertion, hence also the equality $d^{(\mathrm{ab})}\left(G_{\circ}\right)=d^{(\mathrm{ab})}\left(G_{\bullet}\right)$, follows immediately from Proposition 3.4, (iii), and Remark 3.5.1. Thus, the equality $e^{(\mathrm{ab})}\left(G_{\circ}\right)=e^{(\mathrm{ab})}\left(G_{\bullet}\right)$ follows from Proposition 3.4, (iii). This completes the proof of assertion (i).

Next, we verify assertion (ii). The implication $(1) \Rightarrow(2)$ was already discussed in Remark 4.12.1. We verify the implication $(2) \Rightarrow(1)$. Suppose that condition (2) is satisfied. Let us first observe that it follows from Remark 4.12.1 that, to verify the implication $(2) \Rightarrow(1)$, we may assume without loss of generality, by replacing $G_{0}$. by the image of $\alpha$ [which is of MLF-type-cf. the discussion following [3], Proposition 3.3], that $\alpha$ is surjective. Thus, since [we have assumed that] $d\left(G_{\circ}\right)=d^{(\mathrm{ab})}\left(G_{\circ}\right)$, it follows from assertion (i), together with the [easily verified] inequality $d^{(\mathrm{ab})}\left(G_{\bullet}\right) \leq d\left(G_{\bullet}\right)$, that $d\left(G_{\circ}\right) \leq d\left(G_{\bullet}\right)$. In particular, it follows from Theorem 3.6 that $\alpha$ is an isomorphism, as desired. This completes the proof of the implication $(2) \Rightarrow(1)$, hence also of assertion (ii).

Assertion (iii) follows - in light of Proposition 4.9, (iii) - from the [easily verified] fact that an MLF contained in an absolutely abelian MLF is absolutely abelian. This completes the proof of Theorem 4.13.

Corollary 4.14. For each $\square \in\{\circ, \bullet\}$, let $k_{\square}$ be an MLF and $\bar{k}_{\square}$ an algebraic closure of $k_{\square}$; write $G_{\square} \stackrel{\text { def }}{=} \operatorname{Gal}\left(\bar{k}_{\square} / k_{\square}\right)$. Then the following hold: 
(i) Suppose that there exists a cyclotomically compatible surjection $G_{\circ} \rightarrow G_{\bullet}$. Then the field $k_{\circ}^{(\mathrm{ab})}$ is isomorphic to the field $k_{\bullet}^{(\mathrm{ab})}$.

(ii) Suppose that $k_{\circ}$ is absolutely abelian. Then the following three conditions are equivalent:

(1) The field $k_{\circ}$ is isomorphic to the field $k_{\bullet}$.

(2) There exists a cyclotomically compatible surjection $G_{\circ} \rightarrow G_{\bullet}$.

(3) The group $G_{\circ}$ is isomorphic to the group $G_{\bullet}$.

Proof. Assertion (i) follows immediately - in light of Proposition 4.9, (i) - from Theorem 4.13, (i), and [3], Lemma 1.7, (1), (2). Next, we verify assertion (ii). The implication $(1) \Rightarrow(2)$ is immediate. The implication $(2) \Rightarrow(3)$ follows - in light of Proposition 4.9, (iii) - from Theorem 4.13, (ii). Finally, we verify the implication $(3) \Rightarrow(1)$. Suppose that condition (3) is satisfied. Then it follows from Proposition 4.9, (iii), that $k_{\bullet}$ is absolutely abelian. Thus, the implication $(3) \Rightarrow(1)$ follows from assertion (i). This completes the proof of the implication $(3) \Rightarrow(1)$, hence also of assertion (ii).

REMARK 4.14.1. The main theorem of [6] is equivalent to Corollary 4.14, (i), in the case where the surjection " $G_{\circ} \rightarrow G_{\bullet}$ " is an isomorphism. Now let us recall that it is immediate that every isomorphism between groups of MLF-type is cyclotomically compatible. Thus, Corollary 4.14, (i), may be regarded as a refinement of the main theorem of [6].

Some of the group-theoretic reconstruction algorithms discussed in the present $\S 4$ may be summarized as follows.

SUMMARY 4.15.

(i) There exist group-theoretic reconstruction algorithms [cf. [8], Remark 1.9.8] for constructing, from a group $G$ of MLF-type,

- topological rings $\mathbb{Z}_{p}(G) \subseteq \mathbb{Q}_{p}(G)[c f$. Definition 4.5, (ii), (iii)],

- a homomorphism $\mathrm{Nm}_{\mathrm{abs}}(G): k^{\times}(G) \rightarrow \mathbb{Q}_{p}(G)^{\times}$of topological modules [cf. Definition 4.7, (iii)], and

- integers $d^{(\mathrm{ab})}(G)$ and $e^{(\mathrm{ab})}(G)$ [cf. Definition 4.7, (iv)]

which "correspond" to

- the topological rings $\mathbb{Z}_{p_{k}} \subseteq \mathbb{Q}_{p_{k}}[$ cf. Lemma 4.6, (i)],

- the Norm map $\mathrm{Nm}_{k / k^{(d=1)}}: k^{\times} \rightarrow\left(k^{(d=1)}\right)^{\times}$with respect to the finite extension $k / k^{(d=1)}$ [cf. Proposition 4.9, (i)], and respectively.

- the integers $d_{k}^{(\mathrm{ab})}$ and $e_{k}^{(\mathrm{ab})}$ [cf. Proposition 4.9, (ii)],

(ii) There exists a group-theoretic condition for a group of MLF-type [cf. Definition 4.8] which "corresponds" to the condition for an MLF to be absolutely abelian [cf. Proposition 4.9, (iii)]. 
REMARK 4.15.1.

(i) By Summary 4.15, (ii), one may conclude that

the condition for an MLF to be absolutely abelian may be considered to be "group-theoretic".

(ii) On the other hand, it follows from example (1) given in [4], §2 [i.e., " $L_{1}$ " and " $L_{3}$ " in (1) of [4], §2], that there exist an absolutely Galois MLF $k_{\circ}$ and an MLF $k_{\bullet}$ that is not absolutely Galois such that the absolute Galois group of $k_{\circ}$ is isomorphic, as an abstract profinite group, to the absolute Galois group of $k_{\bullet}$. By this fact, one may conclude that

the condition for an MLF to be absolutely Galois should be considered to be "not group-theoretic".

\section{Reconstruction algorithms related to MLF's of degree one}

In the present $\S 5$, we maintain the notational conventions introduced at the beginnings of $\S 1$ and $\S 2$. In particular, we have been given a group of MLF-type

$G$.

In the present $\S 5$, suppose that

$$
d(G)=1 .
$$

In the present $\S 5$, we establish some group-theoretic reconstruction algorithms [cf. [8], Remark 1.9.8] related to MLF's of degree one, i.e., such that the integer " $d_{(-)}$" [cf. the notational conventions introduced at the beginning of $\S 1$ ] is equal to one. As a consequence of the group-theoretic reconstruction algorithms, we also prove [cf. Theorem 5.9, (ii), below] that every absolutely strictly radical [cf. Definition 5.6, (iii), below] MLF is absolutely characteristic [cf. Definition 5.7 below].

LEMMA 5.1. The homomorphism

$$
\operatorname{Nm}_{\mathrm{abs}}(G): k^{\times}(G) \rightarrow \mathbb{Q}_{p}(G)^{\times}
$$

[cf. Definition 4.7, (iii)] is an isomorphism of topological modules.

Proof. Since [we have assumed that] $d(G)=1$, this assertion follows from Proposition 4.9, (i).

Definition 5.2. Consider the isomorphism $k_{\times}(G) \stackrel{\sim}{\rightarrow} \mathbb{Q}_{p}(G)_{\times}$of topological monoids [cf. Remark 2.3.1] determined by the isomorphism $k^{\times}(G) \stackrel{\sim}{\rightarrow}$ $\mathbb{Q}_{p}(G)^{\times}$of Lemma 5.1 [cf. the discussion entitled "Fields" in $\S 0$ ]. Then, by 
means of the topological field structure of $\mathbb{Q}_{p}(G)$, i.e., on $\mathbb{Q}_{p}(G)_{\times}$, together with this isomorphism, one may define a structure of topological field on $k_{\times}(G)$. We shall write

$$
k(G)
$$

for the resulting topological field. Thus, we have a tautological isomorphism of topological fields

$$
k(G) \stackrel{\sim}{\rightarrow} \mathbb{Q}_{p}(G)
$$

and natural identifications

$$
k(G)_{\times}=k_{\times}(G), \quad k(G)^{\times}=k^{\times}(G) .
$$

REMARK 5.2.1. One verifies immediately that the topological field $k(G)$ is isomorphic, as an abstract topological field, to the topological field $\mathbb{Q}_{p(G)}$ [cf. also Lemma 4.6, (i)].

Definition 5.3. Let $H \curvearrowright M$ be an $M^{\circ} F^{\triangleright}$-pair [cf. [3], Definition 5.3] such that $d(H)=1$ [cf. [3], Remark 5.4.1]. Thus, the Kummer polyisomorphism $\kappa(H \curvearrowright M):(H \curvearrowright M) \stackrel{\sim}{\rightarrow}\left(H \curvearrowright M^{\triangleright}(H)=\overline{\mathcal{O}} \triangleright(H)\right)$ [cf. [3], Definition 5.8; [3], Definition 7.4] associated to $H \curvearrowright M$ consists of a single isomorphism [cf. [3], Definition 5.5] [i.e., as opposite to just a polyisomorphism]. Then, by means of the topological field structure of $k(H)$, i.e., on $k_{\times}(H)$, of Definition 5.2, together with the isomorphism $\left(\left(M^{\mathrm{gp}}\right)^{H}\right)^{\circledast} \stackrel{\sim}{\rightarrow}$ $\left(\bar{k}^{\times}(H)^{H}\right)^{\circledast}=k_{\times}(H)$ [cf. [3], Proposition 5.7, (i)] induced by the Kummer polyisomorphism $\kappa(H \curvearrowright M)$ [consisting of a single isomorphism], one may define a structure of topological field on $\left(\left(M^{\mathrm{gp}}\right)^{H}\right)^{\circledast}$. We shall write

$$
k(H \curvearrowright M)
$$

for the resulting topological field. Thus, we have tautological isomorphisms of topological fields

$$
k(H \curvearrowright M) \stackrel{\sim}{\rightarrow} k(H) \stackrel{\sim}{\rightarrow} \mathbb{Q}_{p}(H)
$$

and natural identifications

$$
k(H \curvearrowright M)_{\times}=\left(\left(M^{\mathrm{gp}}\right)^{H}\right)^{\circledast}, \quad k(H \curvearrowright M)^{\times}=\left(M^{\mathrm{gp}}\right)^{H} .
$$

REMARK 5.3.1. One verifies immediately that, in the situation of Definition 5.3, the topological field $k(H \curvearrowright M)$ is isomorphic, as an abstract topological field, to the topological field $\mathbb{Q}_{p(H)}$ [cf. also Lemma 4.6, (i)].

REMARK 5.3.2. Let us recall the "étale-like" $\mathrm{MLF}^{\triangleright}$-pair $G \curvearrowright \overline{\mathcal{O}} \triangleright(G)[\mathrm{cf}$. [3], Definition 5.8]. Then one verifies immediately from the various definitions 
involved that we have a natural identification

$$
k(G)=k\left(G \curvearrowright \overline{\mathcal{O}}^{\triangleright}(G)\right) .
$$

Recall the MLF

$$
k
$$

introduced at the beginning of $\S 1$.

THEOREM 5.4. Suppose that $d_{k}=1$, which thus implies that $d\left(G_{k}\right)=1$ [cf. [3], Proposition 3.6]. Then the following hold:

(i) The homomorphism

$$
\operatorname{rec}_{k}: k^{\times} \hookrightarrow G_{k}^{\mathrm{ab}}
$$

of [3], Lemma 1.7, determines an isomorphism of topological fields

$$
k \stackrel{\sim}{\rightarrow} k\left(G_{k}\right) .
$$

(ii) By applying the reconstruction algorithm of Definition 5.3 to the model MLF $^{\triangleright}$-pair $G_{k} \curvearrowright \mathcal{O}_{\vec{k}}^{\triangleright}$ [cf. [3], Definition 5.2], we obtain a topological field $k\left(G_{k} \curvearrowright \mathcal{O}_{\vec{k}}^{\triangleright}\right)$ whose underlying set may be identified with the underlying set of $k$. Then the topological field structure of $k$ on the underlying set of $k$ coincides, relative to this identification, with the topological field structure of $k\left(G_{k} \curvearrowright \mathcal{O}_{\vec{k}}^{\triangleright}\right)$ on the underlying set of $k$.

Proof. These assertions follow immediately from Proposition 4.9, (i).

COROLlary 5.5. The image of the natural homomorphism $\operatorname{Aut}(G) \rightarrow$ $\operatorname{Aut}\left(G^{\mathrm{ab}}\right)$ is trivial.

Proof. Let $\alpha$ be an automorphism of $G$. Now let us observe that since the subset $k^{\times}(G) \subseteq G^{\mathrm{ab}}$ of $G^{\mathrm{ab}}$ is dense [cf. [3], Lemma 1.7, (1); [3], Proposition 3.11 , (i)], to verify Corollary 5.5, it suffices to verify that the automorphism $k^{\times}(\alpha)$ of $k^{\times}(G)$ induced by $\alpha$ is the identity automorphism. On the other hand, since $k^{\times}(\alpha)$ extends to an automorphism of the topological field $k(G)$, and the topological field $k(G)$ is isomorphic, as an abstract topological field, to the topological field $\mathbb{Q}_{p_{k}}$ [cf. Remark 5.2.1; [3], Proposition 3.6], we conclude that $k^{\times}(\alpha)$ is the identity automorphism, as desired. This completes the proof of Corollary 5.5.

DEFINITION 5.6.

(i) We shall refer to a collection of data

$$
\left(n ; m ; r_{1}, \ldots, r_{m} ; a_{1}, \ldots, a_{m}\right)
$$

consisting of 
- positive integers $n, m, r_{1}, \ldots, r_{m}$ such that $n \in \bigcap_{i=1}^{m} r_{i} \mathbb{Z}$ and

- elements $a_{1}, \ldots, a_{m} \in k^{\times}$of $k^{\times}$

as a strictly radical data for $k$.

(ii) Let $K \subseteq \bar{k}$ be a finite extension of $k$. Then we shall say that the finite extension $K / k$ is strictly radical if there exists a strictly radical data $\left(n ; m ; r_{1}, \ldots, r_{m} ; a_{1}, \ldots, a_{m}\right)$ for $k$ such that

$$
K=k\left(\zeta_{n}, a_{1}^{1 / r_{1}}, \ldots, a_{m}^{1 / r_{m}}\right) \subseteq \bar{k} .
$$

(iii) We shall say that the MLF $k$ is absolutely strictly radical if the finite extension $k / k^{(d=1)}$ is strictly radical.

REMARK 5.6.1. One verifies easily that a strictly radical extension is Galois. In particular, an absolutely strictly radical MLF is absolutely Galois [cf. Definition 4.2, (i)].

DEFINITION 5.7. We shall say that the MLF $k$ is absolutely characteristic if the open subgroup $G_{k} \subseteq \operatorname{Gal}\left(\bar{k} / k^{(d=1)}\right)$ of $\operatorname{Gal}\left(\bar{k} / k^{(d=1)}\right)$ is characteristic [cf. Remark 5.7.1 below].

REMARK 5.7.1. One verifies immediately that the issue of whether or not the MLF $k$ satisfies the condition that the open subgroup $G_{k} \subseteq \operatorname{Gal}\left(\bar{k} / k^{(d=1)}\right)$ of $\operatorname{Gal}\left(\bar{k} / k^{(d=1)}\right)$ is characteristic [cf. Definition 5.7] does not depend on the choice of $\bar{k}$, i.e., depends only on $k$.

REMARK 5.7.2.

(i) Let us recall that since $G$ is topologically finitely generated [cf., e.g., [3], Lemma 1.4, (i)], one verifies easily that the topology of the profinite group $G$ admits a basis of characteristic open subgroups.

(ii) It follows from (i) that there exists a finite extension $K \subseteq \bar{k}$ of $k$ such that the MLF $K$ is absolutely characteristic.

Definition 5.8.

(i) Let $H \curvearrowright M$ be an MLF $^{\triangleright}$-pair such that $d(H)=1$ and $(n ; m$; $\left.r_{1}, \ldots, r_{m} ; a_{1}, \ldots, a_{m}\right)$ a strictly radical data for the MLF $k(H \curvearrowright M)$ of Definition 5.3 [cf. also Remark 5.3.1]. Then we shall write

$$
(H \curvearrowright M)\left(n ; m ; r_{1}, \ldots, r_{m} ; a_{1}, \ldots, a_{m}\right) \subseteq H
$$

for the uniquely determined maximal subgroup of $H$ which acts trivially on the subset of $M^{\text {gp }}$

$$
\left\{a \in M^{\mathrm{gp}} \mid a^{n}=1 \text { or } a^{r_{i}}=a_{i} \text { for some } i \in\{1, \ldots, m\}\right\} .
$$

(ii) Let $\left(n ; m ; r_{1}, \ldots, r_{m} ; a_{1}, \ldots, a_{m}\right)$ be a strictly radical data for the MLF $k(G)$ of Definition 5.2 [cf. also Remark 5.2.1]. Then we shall write 


$$
\begin{aligned}
& G\left(n ; m ; r_{1}, \ldots, r_{m} ; a_{1}, \ldots, a_{m}\right) \\
& \quad \stackrel{\text { def }}{=}\left(G \curvearrowright \overline{\mathcal{O}}^{\triangleright}(G)\right)\left(n ; m ; r_{1}, \ldots, r_{m} ; a_{1}, \ldots, a_{m}\right) \subseteq G
\end{aligned}
$$

[cf. Remark 5.3.2].

THEOREM 5.9. The following hold:

(i) Suppose that $d_{k}=1$, which thus implies that $d\left(G_{k}\right)=1$ [cf. [3], Proposition 3.6]. Let $\left(n ; m ; r_{1}, \ldots, r_{m} ; a_{1}, \ldots, a_{m}\right)$ be a strictly radical data for $k \stackrel{\sim}{\rightarrow} k\left(G_{k}\right)[c f$. Theorem 5.4, (i)]. Then it holds that

$$
G_{k}\left(n ; m ; r_{1}, \ldots, r_{m} ; a_{1}, \ldots, a_{m}\right)=\operatorname{Gal}\left(\bar{k} / k\left(\zeta_{n}, a_{1}^{1 / r_{1}}, \ldots, a_{m}^{1 / r_{m}}\right)\right)
$$

-i.e., as subgroups of $G_{k}$.

(ii) Every absolutely strictly radical $M L F$ is absolutely characteristic $[c f$. also Remark 5.9 .1 below].

Proof. Assertion (i) follows immediately from the various definitions involved. Assertion (ii) follows immediately from assertion (i) and Corollary 5.5. This completes the proof of Theorem 5.9.

REMARK 5.9.1. Note that there exists an absolutely characteristic MLF that is not absolutely strictly radical. Indeed, let us observe that one verifies immediately from Kummer theory that if $k$ is absolutely strictly radical, then the Galois group $\operatorname{Gal}\left(k / k^{(d=1)}\right)$ [cf. Remark 5.6.1] has a structure of extension of an abelian group by an abelian group. Thus, it follows from Remark 5.7.2, (i), that if every absolutely characteristic MLF is absolutely strictly radical, then we conclude that the absolute Galois group $\operatorname{Gal}\left(\bar{k} / k^{(d=1)}\right)$ has a structure of extension of an abelian group by an abelian group-in contradiction to some well-known group-theoretic properties [cf., e.g., [12], Theorem 7.5.12] of the $\operatorname{group} \operatorname{Gal}\left(\bar{k} / k^{(d=1)}\right)$.

\section{REMARK 5.9.2.}

(i) It follows from example (1) given in [4], §2 [i.e., " $L_{1}$ " and " $L_{3}$ " in (1) of [4], §2], that there exist an absolutely strictly radical MLF $k_{\circ}$ and an MLF $k_{\bullet}$ that is not absolutely strictly radical [cf. Remark 5.6.1] such that the absolute Galois group of $k_{\circ}$ is isomorphic, as an abstract profinite group, to the absolute Galois group of $k_{\bullet}$. By this fact, one may conclude that

the condition for an MLF to be absolutely strictly radical should be considered to be "not group-theoretic".

(ii) It follows from example (1) given in [4], $\$ 2$ [i.e., " $L_{1}$ " and " $L_{3}$ " in (1) of [4], §2], that there exist an absolutely characteristic MLF $k_{\circ}$ [cf. Theorem 


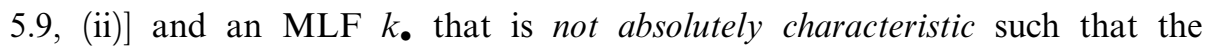
absolute Galois group of $k_{\circ}$ is isomorphic, as an abstract profinite group, to the absolute Galois group of $k_{\bullet}$. By this fact, one may conclude that

the condition for an MLF to be absolutely characteristic should be considered to be "not group-theoretic".

REMARK 5.9.3.

(i) Let us first observe the following "tautological" assertion in the anabelian geometry of absolutely characteristic MLF's:

For each $\square \in\{0, \bullet\}$, let $k_{\square}$ be an absolutely characteristic MLF and $\bar{k}_{\square}$ an algebraic closure of $k_{\square}$; write $G_{\square} \stackrel{\text { def }}{=} \operatorname{Gal}\left(\bar{k}_{\square} / k_{\square}\right)$. Then the following two conditions are equivalent:

(1) The field $k_{\circ}$ is isomorphic to the field $k_{\bullet}$.

(2) There exists an isomorphism $G_{\circ} \stackrel{\sim}{\rightarrow} G_{\bullet}$ compatible with the respective natural outer actions of $\operatorname{Gal}\left(k_{\circ} / k_{\circ}^{(d=1)}\right), \operatorname{Gal}\left(k_{\bullet} / k_{\bullet}^{(d=1)}\right)$ [i.e., by conjugation] relative to some isomorphism $\operatorname{Gal}\left(k_{\circ} / k_{\circ}^{(d=1)}\right) \stackrel{\sim}{\rightarrow}$ $\operatorname{Gal}\left(k_{\bullet} / k_{\bullet}^{(d=1)}\right)$.

[This assertion follows immediately from the definition of the notion of absolutely characteristic MLF-cf. also [3], Proposition 3.6.]

(ii) It follows from Theorem 5.9, (ii), that one may apply the "tautological" assertion of (i) to absolutely strictly radical MLF's.

(iii) Finally, let us observe that it follows from example (1) given in [4], $\S 2$ [i.e., " $L_{1}$ " and " $L_{2}$ " in (1) of [4], §2], that there exist absolutely strictly radical MLF's $k_{\circ}, k_{\bullet}$ such that the field $k_{\circ}$ is not isomorphic to the field $k_{\bullet}$, but the absolute Galois group of $k_{\circ}$ is isomorphic, as an abstract profinite group, to the absolute Galois group of $k_{\bullet}$. Thus, we conclude from Theorem 5.9, (ii), that, in the "tautological" assertion of (i), one cannot replace condition (2) by the following condition:

$\left(2^{\prime}\right)$ There exists an isomorphism $G_{\circ} \stackrel{\sim}{\rightarrow} G_{\bullet}$.

REMARK 5.9.4. Let us recall the following three well-known facts in anabelian geometry:

(1) One verifies easily that an immediate consequence of the NeukirchUchida theorem [cf. the main theorem of [16]] is that every normal open subgroup of the absolute Galois group of the field of rational numbers is characteristic.

(2) It follows immediately from [7], Corollary 3.7, that it holds that the natural injection $\operatorname{Aut}(k) \hookrightarrow \operatorname{Out}\left(G_{k}\right)$ [cf., e.g., [3], Proposition 2.1] is bijective if and only if each member of the filtration on $G_{k}$ given by the higher ramification groups in the upper numbering is characteristic. 
(3) Suppose that $d_{k}=1$. Then it follows immediately from the equivalence of (2) [cf. also the argument in Remark 6.3.1, (ii), below] that the natural injection $\operatorname{Aut}(k) \hookrightarrow \operatorname{Out}\left(G_{k}\right)$ [cf., e.g., [3], Proposition 2.1] is bijective if and only if every normal open subgroup of $G_{k}$ is characteristic. [Note that this equivalence also follows from [5], Theorem A.]

By these facts, one may find the importance of discussing the issue of whether or not a given closed subgroup of the absolute Galois group of a field is characteristic in the study of anabelian geometry. This observation is one of motivations of studying Theorem 5.9, (ii).

Some of the group-theoretic reconstruction algorithms discussed in the present $\S 5$ may be summarized as follows.

SUMmARY 5.10. There exist group-theoretic reconstruction algorithms $[c f$. [8], Remark 1.9.8] for constructing, from a group $G$ of MLF-type such that $d(G)=1$,

- a structure of topological field on $k_{\times}(G)$ [cf. Definition 5.2] and

- a collection of subgroups of $G$ [cf. Definition 5.8, (ii)]

which "correspond" to

- the topological field structure of $k$ on $k_{\times}[c f$. Theorem 5.4, (i)] and

- the collection of open subgroups of $G_{k}$ corresponding to the absolutely strictly radical MLF's contained in $\bar{k}$ [cf. Theorem 5.9, (i)], respectively.

REMARK 5.10.1. Let us recall that, as asserted in Summary 5.10, we have established [cf. Definition 5.2] a group-theoretic reconstruction algorithm for constructing, from a group $G$ of MLF-type such that $d(G)=1$, a structure of topological field on $k_{\times}(G)$ which "corresponds" to the topological field structure of $k$, i.e., on $k_{\times}$.

Here, let us also recall that, as discussed in [2], Remark 1.4.1, (ii), it is impossible to establish a group-theoretic reconstruction algorithm for constructing, from an arbitrary group $G$ of MLF-type, such a structure of topological field on $k_{\times}(G)$.

\section{Reconstruction algorithms related to Galois-specifiable MLF's}

In the present $\S 6$, we maintain the notational conventions introduced at the beginnings of $\S 1$ and $\S 2$. In the present $\S 6$, we consider Galois-specifiable [cf. Definition 6.1 below] MLF's. Moreover, we also establish some grouptheoretic reconstruction algorithms [cf. [8], Remark 1.9.8] related to Galoisspecifiable MLF's. For instance, we establish a group-theoretic reconstruction algorithm for constructing, from a group of MLF-type that satisfies a certain 
condition, a collection of subgroups of the outer automorphism group of the group of MLF-type which "corresponds" to the $\operatorname{Out}\left(G_{k}\right)$-orbit, i.e., by conjugation, of the subgroup of $\operatorname{Out}\left(G_{k}\right)$ determined by the field automorphisms of $k$ [cf. Definition 6.8, (ii); Theorem 6.12, (ii), below].

Definition 6.1. We shall say that the MLF $k$ is Galois-specifiable if the MLF $k$ is absolutely Galois [cf. Definition 4.2, (i)], and, moreover, the following condition is satisfied: If $L$ is an MLF such that there exist an algebraic closure $\bar{L}$ of $L$ and an isomorphism $G_{k} \stackrel{\sim}{\rightarrow} \operatorname{Gal}(\bar{L} / L)$ of groups, then the field $k$ is isomorphic, as an abstract field, to the field $L$ [cf. Remark 6.1.1 below].

REMARK 6.1.1. One verifies immediately that the issue of whether or not the MLF $k$ satisfies the condition in Definition 6.1 does not depend on the choice of $\bar{k}$, i.e., depends only on $k$.

REMARK 6.1.2. Suppose that $\left(p_{k}, a_{k}\right) \neq(2,1)$. Then it follows immediately - in light of Proposition 2.5, (i); Proposition 4.9, (i); [3], Proposition 3.6-from Theorem 4.11, together with [3], Lemma 1.7, (1), (2), that it holds that the MLF $k$ is Galois-specifiable if and only if the following condition is satisfied: If $K \subseteq \bar{k}$ is a finite extension of $k^{(d=1)}$ such that $d_{k}=d_{K}$, and, moreover, $k^{(\mathrm{ab})}=K^{(\mathrm{ab})}$ [cf. Definition 4.2, (iii)], i.e., as subfields of $\bar{k}$, then $k=K$, i.e., as subfields of $\bar{k}$.

LemMA 6.2. Suppose that $k$ is Galois over $k^{(\mathrm{ab})}$. Let $K \subseteq \bar{k}$ be a finite unramified [necessarily Galois] extension of $k^{(\mathrm{ab})}$. Note that it follows immediately from Lemma 4.3, (ii), that we have a natural isomorphism $\operatorname{Gal}\left(k \cdot K / k^{(\mathrm{ab})}\right)$ $\stackrel{\sim}{\rightarrow} \mathrm{Gal}\left(k / k^{(\mathrm{ab})}\right) \times \mathrm{Gal}\left(K / k^{(\mathrm{ab})}\right)$. Let $\phi: \mathrm{Gal}\left(K / k^{(\mathrm{ab})}\right) \rightarrow \mathrm{Gal}\left(k / k^{(\mathrm{ab})}\right)$ be a homomorphism of groups. Write $L$ for the intermediate field of the finite Galois extension $k \cdot K / k^{(\mathrm{ab})}$ which corresponds, relative to the above natural isomorphism $\mathrm{Gal}\left(k \cdot K / k^{(\mathrm{ab})}\right) \stackrel{\sim}{\rightarrow} \mathrm{Gal}\left(k / k^{(\mathrm{ab})}\right) \times \mathrm{Gal}\left(K / k^{(\mathrm{ab})}\right)$, to the graph $\left(\subseteq \operatorname{Gal}\left(k / k^{(\mathrm{ab})}\right) \times \operatorname{Gal}\left(K / k^{(\mathrm{ab})}\right)\right)$ of the homomorphism $\phi$. Then the equalities $d_{L}=d_{k}, L^{(\mathrm{ab})}=k^{(\mathrm{ab})}$ hold.

Proof. The equality $d_{L}=d_{k}$ follows from the fact that the graph $\left(\subseteq \operatorname{Gal}\left(k / k^{(\mathrm{ab})}\right) \times \operatorname{Gal}\left(K / k^{(\mathrm{ab})}\right)\right)$ of the homomorphism $\phi$ is isomorphic, as an abstract group, to the $\operatorname{group} \operatorname{Gal}\left(K / k^{(\mathrm{ab})}\right)$.

To verify the equality $L^{(\mathrm{ab})}=k^{(\mathrm{ab})}$, assume that $L^{(\mathrm{ab})} \neq k^{(\mathrm{ab})}$, i.e., that the extension $L^{(\mathrm{ab})} / k^{(\mathrm{ab})}$ is not of degree one. Then since the intermediate field $L$ corresponds to the graph of $\phi$, one verifies immediately from the natural isomorphism $\mathrm{Gal}\left(k \cdot K / k^{(\mathrm{ab})}\right) \stackrel{\sim}{\rightarrow} \mathrm{Gal}\left(k / k^{(\mathrm{ab})}\right) \times \mathrm{Gal}\left(K / k^{(\mathrm{ab})}\right)$ that the extension $L^{(\mathrm{ab})} \cdot K / K$ is not of degree one, which thus implies that the extension $k \cap\left(L^{(\mathrm{ab})} \cdot K\right) / k^{(\mathrm{ab})}$ is not of degree one. On the other hand, one verifies easily [cf. the proof of Lemma 4.3, (ii)] that the MLF $L^{(\mathrm{ab})} \cdot K$, hence also the MLF 
$k \cap\left(L^{(\mathrm{ab})} \cdot K\right)$, is absolutely abelian [cf. Definition 4.2, (ii)]. Thus, we obtain a contradiction [cf. Lemma 4.3, (i)]. This completes the proof of the equality $L^{(\mathrm{ab})}=k^{(\mathrm{ab})}$, hence also of Lemma 6.2.

THEOREM 6.3. Consider the following four conditions:

(1) The $M L F k$ is absolutely abelian [cf. Definition 4.2, (ii)].

(2) The $M L F k$ is Galois-specifiable.

(3) The $M L F k$ is absolutely characteristic [cf. Definition 5.7].

(4) The MLF $k$ is absolutely Galois [cf. Definition 4.2, (i)]. Then the following hold:

(i) The implications

$$
(1) \Rightarrow(2) \Rightarrow(3) \Rightarrow(4)
$$

hold [cf. also Remark 6.3.1 below].

(ii) Suppose that $\left(p_{k}, a_{k}\right) \neq(2,1)$. Then the equivalence

$$
(1) \Leftrightarrow(2)
$$

holds.

Proof. First, we verify assertion (i). The first implication in assertion (i) follows immediately from Corollary 4.14, (ii). The second and third implications in assertion (i) follow immediately from the various definitions involved. This completes the proof of assertion (i).

Next, we verify assertion (ii). By assertion (i), to verify assertion (ii), it suffices to verify the implication $(2) \Rightarrow(1)$. Suppose that $k$ is Galois-specifiable. To verify that $k$ is absolutely abelian, let $A \subseteq \mathrm{Gal}\left(k / k^{(\mathrm{ab})}\right)$ be a cyclic subgroup of $\operatorname{Gal}\left(k / k^{(\mathrm{ab})}\right)$. Then it follows from [3], Lemma 1.5, (i), that there exist a finite unramified [necessarily Galois] extension $K \subseteq \bar{k}$ of $k^{(\mathrm{ab})}$ and an isomorphism $\phi: \operatorname{Gal}\left(K / k^{(\mathrm{ab})}\right) \stackrel{\sim}{\rightarrow} A\left(\subseteq \operatorname{Gal}\left(k / k^{(\mathrm{ab})}\right)\right)$ of groups. Thus, it follows from Lemma 6.2 that the graph of $\phi$ determines an MLF $L \subseteq k \cdot K$ such that $d_{L}=d_{k}$, and, moreover, $L^{(\mathrm{ab})}=k^{(\mathrm{ab})}$. On the other hand, since [we have assumed that] $k$ is Galois-specifiable, it follows from Remark 6.1.2 that $k=L$, i.e., that the homomorphism $\phi$, hence also the subgroup $A$, is trivial. In particular, we conclude that every cyclic subgroup of $\operatorname{Gal}\left(k / k^{(\mathrm{ab})}\right)$, hence also the group $\operatorname{Gal}\left(k / k^{(\mathrm{ab})}\right)$ itself, is trivial, as desired. This completes the proof of assertion (ii), hence also of Theorem 6.3.

REMARK 6.3.1. Suppose that we are in the situation of Theorem 6.3.

(i) In general, the implication $(3) \Rightarrow(2)$ does not hold. Indeed, for an odd prime number $p_{k}$, the MLF $\mathbb{Q}_{p_{k}}\left(\zeta_{p_{k}}, p_{k}^{1 / p_{k}}\right)$ is absolutely characteristic [cf. Theorem 5.9, (ii)] but not Galois-specifiable [cf. example (1) given in [4], $\S 2]$. 
(ii) In general, the implication (4) $\Rightarrow(3)$ does not hold. Indeed, let us first observe that it is immediate that the implication $(4) \Rightarrow(3)$ is equivalent to the following assertion:

Every normal open subgroup of $G_{k^{(d=1)}} \stackrel{\text { def }}{=} \mathrm{Gal}\left(\bar{k} / k^{(d=1)}\right)$ is characteristic.

Thus, since every normal closed subgroup of $G_{k^{(d=1)}}$ may be obtained as the intersection of the normal open subgroups of $G_{k^{(d=1)}}$ that contain the normal closed subgroup, if the implication (4) $\Rightarrow(3)$ holds, then we conclude that every normal closed subgroup of $G_{k^{(d=1)}}$ is characteristic. In particular, if the implication $(4) \Rightarrow(3)$ holds, then it follows from [7], Corollary 3.7, that every outer automorphism of $G_{k^{(d=1)}}$ arises from an automorphism of the field $k^{(d=1)}$, i.e., that $\operatorname{Out}\left(G_{k^{(d=1)}}\right)=\{1\}$. But this contradicts the conclusion of the discussion given at the final portion of [12], Chapter VII, $\S 5$, if $p_{k}$ is odd.

Definition 6.4. Recall that the natural homomorphism $\operatorname{Aut}(k) \rightarrow$ $\operatorname{Out}\left(G_{k}\right)$ is injective [cf., e.g., [3], Proposition 2.1]. By means of this injection, let us regard $\operatorname{Aut}(k)$ as a [necessarily finite] subgroup of $\operatorname{Out}\left(G_{k}\right)$ :

$$
\operatorname{Aut}(k) \subseteq \operatorname{Out}\left(G_{k}\right)
$$

Then we shall write

$$
\operatorname{OrbAut}(k)
$$

for the set of $\operatorname{Out}\left(G_{k}\right)$-conjugates of the subgroup $\operatorname{Aut}(k) \subseteq \operatorname{Out}\left(G_{k}\right)$, i.e., the $\operatorname{Out}\left(G_{k}\right)$-orbit of the subgroup $\operatorname{Aut}(k) \subseteq \operatorname{Out}\left(G_{k}\right)$.

Recall the group of MLF-type

\section{G}

introduced at the beginning of $\S 2$.

Definition 6.5. Let $\Gamma \subseteq \operatorname{Out}(G)$ be a finite subgroup of the outer automorphism group $\operatorname{Out}(G)$ of $G$.

(i) We shall write

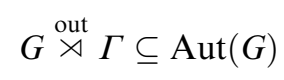

for the inverse image of $\Gamma \subseteq \operatorname{Out}(G)$ by the natural surjection $\operatorname{Aut}(G) \rightarrow$ $\operatorname{Out}(G)$. Thus, since $G$ may be identified with $\operatorname{Inn}(G) \subseteq \operatorname{Aut}(G)$ by the natural isomorphism $G \stackrel{\sim}{\rightarrow} \operatorname{Inn}(G)$ [cf. [3], Lemma 1.8], the group $G \stackrel{\text { out }}{\rtimes} \Gamma$ has a natural structure of extension of $\Gamma$ by $G$ :

$$
1 \rightarrow G \rightarrow G \stackrel{\text { out }}{\rtimes} \Gamma \rightarrow \Gamma \rightarrow 1
$$


By means of the second arrow of this exact sequence, let us always regard $G$ as a subgroup of $G \stackrel{\text { out }}{\rtimes} \Gamma$ :

$$
G \subseteq G \stackrel{\text { out }}{\rtimes} \Gamma
$$

(ii) We shall say that the finite subgroup $\Gamma$ is quasi-geometric if the group $G \stackrel{\text { out }}{\rtimes} \Gamma$ is of MLF-type.

(iii) We shall say that the finite subgroup $\Gamma$ is strictly quasi-geometric if $\Gamma$ is quasi-geometric, and, moreover, the equality $d(G \stackrel{\text { out }}{\rtimes} \Gamma)=1$ holds.

Lemma 6.6. Let $\Gamma \subseteq \operatorname{Out}(G)$ be a quasi-geometric subgroup of $\operatorname{Out}(G)$. Then the following hold:

(i) Every subgroup of $\Gamma$ is a quasi-geometric subgroup of $\operatorname{Out}(G)$.

(ii) The natural inclusion $k^{\times}(G$ out $\Gamma) \hookrightarrow k^{\times}(G)-i . e$., determined by the transfer map with respect to $G \subseteq G \rtimes \Gamma$ [cf. [3], Lemma 1.7, (3)]- and the natural action of $\Gamma$ on $k^{\times}(G)$ determine an isomorphism $k^{\times}(G \stackrel{\text { out }}{\rtimes} \Gamma) \stackrel{\sim}{\rightarrow} k^{\times}(G)^{\Gamma}$.

(iii) It holds that $d(G) \in \# \Gamma \mathbb{Z}$.

(iv) It holds that $\Gamma$ is strictly quasi-geometric if and only if $\# \Gamma=d(G)$.

Proof. Assertion (i) follows from the discussion following [3], Proposition 3.3. Assertion (ii) follows immediately from [3], Proposition 3.11, (i). Assertions (iii), (iv) follow immediately from [3], Proposition 3.6. This completes the proof of Lemma 6.6.

Proposition 6.7. The subgroup $\operatorname{Aut}(k) \subseteq \operatorname{Out}\left(G_{k}\right)$ is quasi-geometric. If, moreover, the $M L F k$ is absolutely Galois, then the $\operatorname{subgroup} \operatorname{Aut}(k) \subseteq \operatorname{Out}\left(G_{k}\right)$ is strictly quasi-geometric.

Proof. This assertion follows immediately from the various definitions involved.

DEFINITION 6.8.

(i) We shall say that $G$ is of GSMLF-type if the following two conditions are satisfied:

(1) There exists a strictly quasi-geometric subgroup of $\operatorname{Out}(G)$.

(2) For each strictly quasi-geometric subgroup $\Gamma \subseteq \operatorname{Out}(G)$ of $\operatorname{Out}(G)$ and each open subgroup $H \subseteq G \stackrel{\text { out }}{\rtimes} \Gamma$ of $G \stackrel{\text { out }}{\rtimes} \Gamma$, if $H$ is isomorphic, as an abstract group, to $G$, then $H=G$, i.e., as subgroups of $G \rtimes \Gamma$ out

[Here, "GSMLF" is to be understood as an abbreviation for "Galois-specifiable mixed-characteristic local field"-cf. Theorem 6.10 below.]

(ii) Suppose that $G$ is of GSMLF-type. Then we shall write

$$
\operatorname{Orb}_{\text {sqg }}(G)
$$

for the set of strictly quasi-geometric subgroups of $\operatorname{Out}(G)$. 
LEMMA 6.9. Let $\overline{\mathbb{Q}}_{p_{k}}$ be an algebraic closure of $k^{(d=1)}$. Let us fix an isomorphism $\imath: \bar{k} \stackrel{\sim}{\rightarrow} \overline{\mathbb{Q}}_{p_{k}}$ of fields. Write $k_{l} \stackrel{\text { def }}{=} \imath(k) \subseteq \overline{\mathbb{Q}}_{p_{k}}$ for the finite extension of $k^{(d=1)}$ obtained by forming the image of $k \subseteq \bar{k}$ by $l$. Let $\Gamma \subseteq \operatorname{Out}\left(G_{k}\right)$ be a strictly quasi-geometric subgroup of the outer automorphism group $\operatorname{Out}\left(G_{k}\right)$ of the group $G_{k}$ of MLF-type. Then the following hold:

(i) There exists an isomorphism $\alpha: G_{k} \stackrel{\text { out }}{\rtimes} \stackrel{\sim}{\rightarrow} \operatorname{Gal}\left(\overline{\mathbb{Q}}_{p_{k}} / k^{(d=1)}\right)$ of groups.

(ii) Suppose either that the MLF $k$ is Galois-specifiable, or that the group $G_{k}$ is of GSMLF-type. Then the isomorphism $\alpha$ of (i) restricts to an isomorphism between the subgroup $G_{k} \subseteq G_{k} \stackrel{\text { out }}{\rtimes} \Gamma$ with the subgroup $\operatorname{Gal}\left(\overline{\mathbb{Q}}_{p_{k}} / k_{l}\right) \subseteq$ $\operatorname{Gal}\left(\overline{\mathbb{Q}}_{p_{k}} / k^{(d=1)}\right)$.

Proof. Assertion (i) follows immediately from the definition of the notion of strictly quasi-geometric subgroup [cf. also [3], Proposition 3.6].

Next, we verify assertion (ii) in the case where the MLF $k$ is Galoisspecifiable. Suppose that $k$ is Galois-specifiable. Write $K \subseteq \overline{\mathbb{Q}}_{p_{k}}$ for the finite extension of $k^{(d=1)}$ that corresponds to the open subgroup $\alpha\left(G_{k}\right) \subseteq$ $\operatorname{Gal}\left(\overline{\mathbb{Q}}_{p_{k}} / k^{(d=1)}\right)$ of $\operatorname{Gal}\left(\overline{\mathbb{Q}}_{p_{k}} / k^{(d=1)}\right)$. [So we have a natural identification $\operatorname{Gal}\left(\overline{\mathbb{Q}}_{p_{k}} / K\right)=\alpha\left(G_{k}\right)$.] Thus, since $k$ is Galois-specifiable, we conclude that $k$ is isomorphic, as an abstract field, to $K$. In particular, since $k$ is absolutely Galois, we conclude that $k_{l}=K$, i.e., as subfields of $\overline{\mathbb{Q}}_{p_{k}}$, as desired. This completes the proof of assertion (ii) in the case where the MLF $k$ is Galoisspecifiable.

Finally, we verify assertion (ii) in the case where the group $G_{k}$ is of GSMLF-type. Suppose that $G_{k}$ is of GSMLF-type. Let us first observe that it is immediate that the group $G_{k}$ is isomorphic, as an abstract group, to the group $\operatorname{Gal}\left(\overline{\mathbb{Q}}_{p_{k}} / k_{l}\right) \subseteq \operatorname{Gal}\left(\overline{\mathbb{Q}}_{p_{k}} / k^{(d=1)}\right)$, hence also to the group $\alpha^{-1}\left(\operatorname{Gal}\left(\overline{\mathbb{Q}}_{p_{k}} / k_{l}\right)\right)$ $\subseteq G_{k} \rtimes \Gamma$. Thus, since $G_{k}$ is of GSMLF-type, one may conclude that $G_{k}=$ $\alpha^{-1}\left(\operatorname{Gal}\left(\overline{\mathbb{Q}}_{p_{k}} / k_{l}\right)\right)$, i.e., as subgroups of $G_{k} \stackrel{\text { out }}{\rtimes} \Gamma$, as desired. This completes the proof of assertion (ii) in the case where the group $G_{k}$ is of GSMLF-type, hence also of Lemma 6.9 .

THEOREM 6.10. It holds that the MLF $k$ is Galois-specifiable if and only if the group $G_{k}$ is of GSMLF-type.

Proof. First, we verify the necessity. Suppose that the MLF $k$ is Galoisspecifiable. To verify that the group $G_{k}$ is of $G S M L F$-type, let $\Gamma \subseteq \operatorname{Out}\left(G_{k}\right)$ be a strictly quasi-geometric subgroup of $\operatorname{Out}\left(G_{k}\right)$ [cf. Theorem 6.3, (i); Prop-

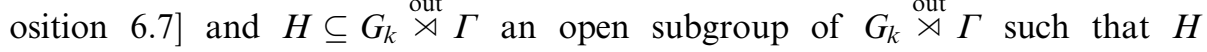
is isomorphic, as an abstract group, to $G_{k}$. Now suppose that we are in the situation of Lemma 6.9. Thus, we have an isomorphism $\alpha: G_{k} \stackrel{\text { out }}{\rtimes} \Gamma \stackrel{\sim}{\rightarrow}$ $\operatorname{Gal}\left(\overline{\mathbb{Q}}_{p_{k}} / k^{(d=1)}\right)$ of groups [cf. Lemma 6.9, (i)] that restricts to an isomorphism $G_{k} \stackrel{\sim}{\rightarrow} \operatorname{Gal}\left(\overline{\mathbb{Q}}_{p_{k}} / k_{l}\right)$ [cf. Lemma 6.9, (ii)]. Write $K \subseteq \overline{\mathbb{Q}}_{p_{k}}$ for the finite extension 
of $k^{(d=1)}$ which corresponds to the open subgroup $\alpha(H) \subseteq \operatorname{Gal}\left(\overline{\mathbb{Q}}_{p_{k}} / k^{(d=1)}\right)$ of $\operatorname{Gal}\left(\overline{\mathbb{Q}}_{p_{k}} / k^{(d=1)}\right)$. [So we have a natural identification $\operatorname{Gal}\left(\overline{\mathbb{Q}}_{p_{k}} / K\right)=\alpha(H)$.] Thus, since $H$ is isomorphic to both $G_{k}$ and $\operatorname{Gal}\left(\overline{\mathbb{Q}}_{p_{k}} / K\right)$, and $k$ is Galoisspecifiable, we conclude that $k$ is isomorphic, as an abstract field, to $K$. In particular, since $k$ is absolutely Galois, it holds that $k_{l}=K$, i.e., as subfields of $\overline{\mathbb{Q}}_{p_{k}}$, which thus implies that $\alpha\left(G_{k}\right)=\alpha(H)$, i.e., as subgroups of $\operatorname{Gal}\left(\overline{\mathbb{Q}}_{p_{k}} / k^{(d=1)}\right)$, as desired. This completes the proof of the necessity.

Next, we verify the sufficiency. Suppose that the group $G_{k}$ is of GSMLFtype. To verify that the MLF $k$ is Galois-specifiable, let $L$ be an MLF and $\bar{L}$ an algebraic closure of $L$ such that $G_{k}$ is isomorphic, as an abstract group, to $\operatorname{Gal}(\bar{L} / L)$. Now take a strictly quasi-geometric subgroup $\Gamma \subseteq \operatorname{Out}\left(G_{k}\right)$ of $\operatorname{Out}\left(G_{k}\right)$ [cf. condition (1) of Definition 6.8, (i)], and suppose that we are in the situation of Lemma 6.9. Thus, we have an isomorphism $\alpha: G_{k} \stackrel{\text { out }}{\rtimes} \Gamma \stackrel{\sim}{\rightarrow}$ $\operatorname{Gal}\left(\overline{\mathbb{Q}}_{p_{k}} / k^{(d=1)}\right)$ of groups [cf. Lemma 6.9, (i)] that restricts to an isomorphism $G_{k} \stackrel{\sim}{\rightarrow} \operatorname{Gal}\left(\overline{\mathbb{Q}}_{p_{k}} / k_{l}\right)$ [cf. Lemma 6.9, (ii)]—which thus implies that the MLF $k_{l}$, hence also the MLF $k$, is absolutely Galois. Then let us observe that it follows from [3], Proposition 3.6, that $\bar{L}$ is isomorphic, as an abstract field, to $\overline{\mathbb{Q}}_{p_{k}}$. Let us identify $\bar{L}$ with $\overline{\mathbb{Q}}_{p_{k}}$ by means of some fixed isomorphism of $\bar{L}$ with $\overline{\mathbb{Q}}_{p_{k}}$. Then since $G_{k}$ is isomorphic to $\operatorname{Gal}(\bar{L} / L)=\operatorname{Gal}\left(\overline{\mathbb{Q}}_{p_{k}} / L\right)$, and $G_{k}$ is of GSMLF-type, we conclude that $G_{k}=\alpha^{-1}\left(\operatorname{Gal}\left(\overline{\mathbb{Q}}_{p_{k}} / L\right)\right)$, i.e., as subgroups of $G_{k} \stackrel{\text { out }}{\rtimes} \Gamma$, which thus implies that $k_{l}=L$, i.e., as subfields of $\overline{\mathbb{Q}}_{p_{k}}$. Thus, the field $k$ is isomorphic to the field $L$, as desired. This completes the proof of the sufficiency, hence also of Theorem 6.10 .

COROLlaRY 6.11. The following hold:

(i) If the group $G$ is of AAMLF-type [cf. Definition 4.8], then $G$ is of GSMLF-type.

(ii) Suppose that $(p(G), a(G)) \neq(2,1)[c f$. Definition 2.4, (ii)]. Then if the group $G$ is of GSMLF-type, then $G$ is of AAMLF-type.

Proof. These assertions follow-in light of Proposition 2.5, (i), and [3], Proposition 3.6-from Theorem 6.3, (i), (ii), together with Proposition 4.9, (iii), and Theorem 6.10.

THEOREM 6.12. Suppose that the MLF $k$ is Galois-specifiable, which thus implies that the group $G_{k}$ is of GSMLF-type [cf. Theorem 6.10]. Then the following hold:

(i) Let $\Gamma_{1}, \Gamma_{2} \subseteq \operatorname{Out}\left(G_{k}\right)$ be strictly quasi-geometric subgroups of $\operatorname{Out}\left(G_{k}\right)$. Then $\Gamma_{1}$ is an $\operatorname{Out}\left(\boldsymbol{G}_{\boldsymbol{k}}\right)$-conjugate of $\Gamma_{2}$.

(ii) It holds that

$$
\operatorname{OrbAut}(k)=\operatorname{Orb}_{\text {sqg }}\left(G_{k}\right) \text {. }
$$


Proof. First, we verify assertion (i). Let $\alpha: G_{k} \stackrel{\text { out }}{\rtimes} \Gamma_{1} \stackrel{\sim}{\rightarrow} G_{k} \stackrel{\text { out }}{\rtimes} \Gamma_{2}$ be an isomorphism of groups [cf. Lemma 6.9, (i)]. Then since the group $G_{k}$ is of GSMLF-type, it is immediate that the isomorphism $\alpha$ restricts to an automorphism of $G_{k}$. Moreover, one verifies immediately from the various definitions involved that $\Gamma_{1}$ is the conjugate, by the outer automorphism of $G_{k}$ determined by the resulting automorphism of $G_{k}$, of $\Gamma_{2}$. This completes the proof of assertion (i).

Assertion (ii) follows from assertion (i) and Proposition 6.7. This completes the proof of Theorem 6.12.

REMARK 6.12.1. Note that, in general, a similar assertion to Theorem 6.12, (i), for [the absolute Galois group of] an absolutely characteristic MLF does not hold [cf. Remark 8.6.1, (ii), below].

Some of the group-theoretic reconstruction algorithms discussed in the present $\S 6$ may be summarized as follows.

SUMMARY 6.13 .

(i) There exists a group-theoretic condition for a group of MLF-type [cf. Definition 6.8, (i)] which "corresponds" to the condition for an MLF to be Galois-specifiable [cf. Theorem 6.10].

(ii) There exists a group-theoretic reconstruction algorithm [cf. [8], Remark 1.9.8] for constructing, from a group $G$ of MLF-type that satisfies the condition of (i), a collection $\operatorname{Orb}_{\mathrm{sqg}}(G)$ of subgroups of $\operatorname{Out}(G)$ [cf. Definition 6.8, (ii)] which "corresponds" to the $\operatorname{Out}\left(G_{k}\right)$-orbit $\operatorname{OrbAut}(k)$, i.e., by conjugation, of the subgroup $\operatorname{Aut}(k) \subseteq \operatorname{Out}\left(G_{k}\right)$ [cf. Theorem 6.12, (ii)].

REMARK 6.13.1. By Summary 6.13, (i), one may conclude that

the condition for an MLF to be Galois-specifiable may be considered to be "group-theoretic".

\section{On outer automorphisms arising from field automorphisms I}

In the present $\S 7$, we maintain the notational conventions introduced at the beginnings of $\S 1$ and $\S 2$. In particular, we have a natural open injection

$$
G_{k} \hookrightarrow G_{k^{(d=1)}} \stackrel{\text { def }}{=} \operatorname{Gal}\left(\bar{k} / k^{(d=1)}\right) .
$$

In the present $\S 7$, we discuss outer automorphisms of the absolute Galois groups of MLF's that arise from field automorphisms of the MLF's. We prove that if the MLF $k$ is absolutely characteristic, and that $p_{k}$ is odd, then the 
subgroup of the outer automorphism group of $G_{k}$ determined by the field automorphisms of $k$ is not normally terminal [cf. Theorem 7.2, (i), below]. Moreover, we also prove that, under some conditions, the outer automorphism group of $G_{k}$ has "many" finite abelian subgroups [cf. Theorem 7.5, Remark 7.5.1, below].

Lemma 7.1. Let $H \subseteq G$ be a characteristic open subgroup of the group $G$ of MLF-type. Thus, we have, by considering restrictions, a natural homomorphism

$$
\operatorname{Aut}(G) \rightarrow \operatorname{Aut}(H)
$$

Then the following hold:

(i) The homomorphism $\operatorname{Aut}(G) \rightarrow \operatorname{Aut}(H)$ is injective. In particular, we also have an injection $\operatorname{Aut}(G) / \operatorname{Inn}(H) \hookrightarrow \operatorname{Out}(H)$. Let us regard $\operatorname{Aut}(G)$, $\operatorname{Aut}(G) / \operatorname{Inn}(H)$ as subgroups of $\operatorname{Aut}(H), \operatorname{Out}(H)$ by means of these injections, respectively:

$$
\operatorname{Aut}(G) \subseteq \operatorname{Aut}(H), \quad \operatorname{Aut}(G) / \operatorname{Inn}(H) \subseteq \operatorname{Out}(H)
$$

(ii) The natural homomorphisms $G \rightarrow \operatorname{Inn}(G) \hookrightarrow \operatorname{Aut}(G)$ determine an isomorphism

$$
G / H \stackrel{\sim}{\rightarrow} \operatorname{Inn}(G) / \operatorname{Inn}(H) .
$$

Let us identify $G / H$ with $\operatorname{Inn}(G) / \operatorname{Inn}(H)$ by means of this isomorphism:

$$
G / H=\operatorname{Inn}(G) / \operatorname{Inn}(H) \quad(\subseteq \operatorname{Aut}(G) / \operatorname{Inn}(H) \subseteq \operatorname{Out}(H)) .
$$

Thus, we have a natural exact sequence

$$
1 \rightarrow G / H \rightarrow \operatorname{Aut}(G) / \operatorname{Inn}(H) \rightarrow \operatorname{Out}(G) \rightarrow 1 .
$$

(iii) It holds that

$$
N_{\text {Out }(H)}(G / H)=\operatorname{Aut}(G) / \operatorname{Inn}(H) .
$$

(iv) Recall the exact sequence

$$
1 \rightarrow G / H \rightarrow \operatorname{Aut}(G) / \operatorname{Inn}(H) \rightarrow \operatorname{Out}(G) \rightarrow 1
$$

of (ii). Then the composite

$$
\operatorname{Aut}(G) / \operatorname{Inn}(H) \rightarrow \operatorname{Aut}(G / H) \rightarrow \operatorname{Out}(G / H)
$$

- where the first arrow is the action by conjugation via the second arrow of the above exact sequence, and the second arrow is the natural surjection-coincides 
with the composite

$$
\operatorname{Aut}(G) / \operatorname{Inn}(H) \rightarrow \operatorname{Out}(G) \rightarrow \operatorname{Out}(G / H)
$$

- where the first arrow is the third arrow of the above exact sequence, and the second arrow is the natural homomorphism.

Proof. First, we verify assertion (i). Let us first observe that it follows from [3], Lemma 1.8, that the action of $G$ on $H$ by conjugation is faithful. Since [it is immediate that] the resulting injection $G \hookrightarrow \operatorname{Aut}(H)$ is $\operatorname{Aut}(G)$ equivariant, assertion (i) holds. This completes the proof of assertion (i). Assertion (ii) follows from [3], Lemma 1.8. Assertions (iii), (iv) follow immediately from the various definitions involved. This completes the proof of Lemma 7.1.

THEOREM 7.2. Suppose that the MLF $k$ is absolutely characteristic [cf. Definition 5.7]. Then the following hold:

(i) Suppose, moreover, that $p_{k}$ is odd. Then the subgroup $\operatorname{Aut}(k) \subseteq$ $\operatorname{Out}\left(G_{k}\right)$ of $\operatorname{Out}\left(G_{k}\right)$ is not normally terminal.

(ii) It holds that the MLF $k$ is absolutely abelian [cf. Definition 4.2, (ii)] if and only if

$$
N_{\text {Out }\left(G_{k}\right)}(\operatorname{Aut}(k))=Z_{\text {Out }\left(G_{k}\right)}(\operatorname{Aut}(k)) .
$$

Proof. Since the open subgroup $G_{k} \subseteq G_{k^{(d=1)}}$ of $G_{k^{(d=1)}}$ is characteristic, by applying Lemma 7.1, (ii), (iii) [in the case where we take the " $H \subseteq G$ " of Lemma 7.1 to be $G_{k} \subseteq G_{k^{(d=1)}}$, we obtain an exact sequence

$$
1 \rightarrow \operatorname{Aut}(k) \rightarrow N_{\operatorname{Out}\left(G_{k}\right)}(\operatorname{Aut}(k)) \rightarrow \operatorname{Out}\left(G_{k^{(d=1)}}\right) \rightarrow 1 .
$$

Now we verify assertion (i). Since $p_{k}$ is odd, it follows from the discussion given at the final portion of [12], Chapter VII, $\S 5$, that $\operatorname{Out}\left(G_{k^{(d=1)}}\right)$ is nontrivial. Thus, by the above exact sequence, we conclude that the subgroup $\operatorname{Aut}(k) \subseteq \operatorname{Out}\left(G_{k}\right)$ is not normally terminal, as desired. This completes the proof of assertion (i).

Next, we verify assertion (ii). The sufficiency is immediate. Let us verify the necessity. Suppose that the MLF $k$ is absolutely abelian. Let us first observe that it is immediate that, to verify the necessity, it suffices to verify that the action of $N_{\operatorname{Out}\left(G_{k}\right)}(\operatorname{Aut}(k))$ on $\operatorname{Aut}(k)$ by conjugation is trivial. On the other hand, since $k$ is absolutely abelian, it follows immediately from Lemma 7.1, (iv), that this action factors through the natural homomorphism $\operatorname{Out}\left(G_{k^{(d=1)}}\right) \rightarrow \operatorname{Out}\left(G_{k^{(d=1)}}^{\mathrm{ab}}\right) \quad\left(=\operatorname{Aut}\left(G_{k^{(d=1)}}^{\mathrm{ab}}\right)\right)$. Thus, the desired triviality follows from Corollary 5.5. This completes the proof of assertion (ii), hence also of Theorem 7.2. 
Remark 7.2.1.

(i) Let us observe that it follows immediately from Corollary 5.5 that if $d_{k}=1$, then the image of the natural homomorphism

$$
\operatorname{Aut}(k) \rightarrow \operatorname{Aut}\left(k_{+}\right)
$$

coincides with the image of the composite

$$
\operatorname{Out}\left(G_{k}\right) \rightarrow \operatorname{Aut}\left(k_{+}\left(G_{k}\right)\right) \stackrel{\operatorname{Aut}\left(k_{+}\right)}{\leftarrow}
$$

- where the second arrow is the isomorphism obtained by conjugation by the vertical isomorphism $k_{+} \stackrel{\sim}{\rightarrow} k_{+}\left(G_{k}\right)$ in the diagram of [3], Proposition 3.11, (iv).

(ii) On the other hand, in general, the image of the natural homomorphism

$$
\operatorname{Aut}(k) \rightarrow \operatorname{Aut}\left(k_{+}\right)
$$

does not coincide with the image of the composite

$$
\operatorname{Out}\left(G_{k}\right) \rightarrow \operatorname{Aut}\left(k_{+}\left(G_{k}\right)\right) \stackrel{\operatorname{Aut}\left(k_{+}\right) .}{\leftarrow}
$$

Indeed, suppose that $k$ is absolutely characteristic, and that the image of the natural homomorphism $\operatorname{Out}\left(G_{k^{(d=1)}}\right) \rightarrow \operatorname{Out}\left(G_{k^{(d=1)}} / G_{k}\right)$ is nontrivial, which thus implies [cf. Lemma 7.1, (iv); also the exact sequence in the proof of Theorem 7.2] that the normal subgroup $\operatorname{Aut}(k) \subseteq N_{\text {Out }\left(G_{k}\right)}(\operatorname{Aut}(k))$ is not a direct summand. [Note that it follows immediately from the discussion given at the final portion of [12], Chapter VII, $\$ 5$, together with Remark 5.7.2, (i), that such a " $k$ " exists.] Next, observe that since $\operatorname{Aut}(k)$ is contained in $N_{\text {Out }\left(G_{k}\right)}(\operatorname{Aut}(k))$, it is immediate that, to verify the desired assertion, it suffices to verify that the image of the natural homomorphism

$$
\operatorname{Aut}(k) \rightarrow \operatorname{Aut}\left(k_{+}\right)
$$

does not coincide with the image of the composite

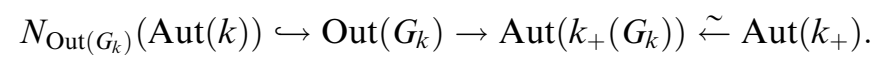

On the other hand, since the natural homomorphism $\operatorname{Aut}(k) \rightarrow \operatorname{Aut}\left(k_{+}\right)$is injective, if these images coincide, then one verifies immediately that the normal subgroup $\operatorname{Aut}(k) \subseteq N_{\operatorname{Out}\left(G_{k}\right)}(\operatorname{Aut}(k))$ is a direct summand-in contradiction to our assumption on $k$.

REMARK 7.2.2. The consideration in Remark 7.2.1, (ii), leads us to, for instance, the following assertion:

If $d_{k}=2$ [which thus implies that $k$ is absolutely abelian], and $p_{k}-1 \notin$ $4 \mathbb{Z}$, then the subgroup $\operatorname{Aut}(k) \subseteq N_{\text {Out }\left(G_{k}\right)}(\operatorname{Aut}(k)) \quad\left(=Z_{\text {Out }\left(G_{k}\right)}(\operatorname{Aut}(k))\right.$

- cf. Theorem 7.2, (ii)) is a direct summand. 
Indeed, let us first observe that it follows from elementary field theory that the action of the [unique] nontrivial element of $\operatorname{Aut}(k)$ on the $\mathbb{Q}_{p_{k}}$-vector space $k_{+}\left(G_{k}\right)\left(\check{\leftarrow} k_{+}-\mathrm{cf}\right.$. [3], Proposition 3.11, (iv)) of dimension $d_{k}=2$ has two eigenvalues $1,-1$. Write $V_{1}, V_{-1} \subseteq k_{+}\left(G_{k}\right)$ for the eigenspaces that corresponds to the eigenvalues $1,-1$, respectively. [So we have $k_{+}\left(G_{k}\right)=V_{1} \oplus$ $V_{-1}$.] Then one verifies easily that the action of $N_{\operatorname{Out}\left(G_{k}\right)}(\operatorname{Aut}(k))$ on $k_{+}\left(G_{k}\right)$ preserves each of the subspaces $V_{1}, V_{-1} \subseteq k_{+}\left(G_{k}\right)$. Thus, we have a homomorphism

$$
N_{\text {Out }\left(G_{k}\right)}(\operatorname{Aut}(k)) \rightarrow \operatorname{Aut}_{\mathbb{Q}_{p_{k}}}\left(V_{-1}\right)=\mathbb{Q}_{p_{k}}^{\times} .
$$

Now let us observe that it follows from our assumption that $p_{k}-1 \notin 4 \mathbb{Z}$, together with [3], Lemma 1.2, (i) [cf. also Proposition 1.1, (v), if $p_{k}=2$ ], that there exists a surjection $\mathbb{Q}_{p_{k}}^{\times} \rightarrow(\mathbb{Z} / 2 \mathbb{Z})_{+}$of modules such that the composite $\{ \pm 1\} \hookrightarrow \mathbb{Q}_{p_{k}}^{\times} \rightarrow(\mathbb{Z} / 2 \mathbb{Z})_{+}$is an isomorphism. Thus, by considering the composite

$$
\operatorname{Aut}(k) \hookrightarrow N_{\operatorname{Out}\left(G_{k}\right)}(\operatorname{Aut}(k)) \rightarrow \operatorname{Aut}_{\mathbb{Q}_{p_{k}}}\left(V_{-1}\right)=\mathbb{Q}_{p_{k}}^{\times} \rightarrow(\mathbb{Z} / 2 \mathbb{Z})_{+},
$$

we conclude that the subgroup $\operatorname{Aut}(k) \subseteq N_{\operatorname{Out}\left(G_{k}\right)}(\operatorname{Aut}(k))$ is a direct summand, as desired.

Lemma 7.3. Suppose that $\left(p_{k}, a_{k}\right) \neq(2,1)$. Write $\mathrm{Nm}_{k / k^{(d=1)}}^{\wedge}:\left(k^{\times}\right)^{\wedge} \rightarrow$ $\left(\left(k^{(d=1)}\right)^{\times}\right)^{\wedge}$ for the [necessarily open] homomorphism of abelian profinite groups induced by the Norm map $\mathrm{Nm}_{k / k^{(d=1)}}: k^{\times} \rightarrow\left(k^{(d=1)}\right)^{\times}$. Then the following hold:

(i) The image of the [uniquely determined] pro- $p_{k}$-Sylow subgroup of the abelian profinite group $\left(k^{\times}\right)^{\wedge}$ by $\mathrm{Nm}_{k / k^{(d=1)}}^{\wedge}$ is a free $\mathbb{Z}_{p_{k}}$-module of rank two.

(ii) There exists a pro- $p_{k}$ closed subgroup $M \subseteq \operatorname{Ker}\left(\mathrm{Nm}_{k / k^{(d=1)}}^{\wedge}\right)$ of the kernel of $\mathrm{Nm}_{k / k^{(d=1)}}^{\wedge}$ such that $M$ is a free $\mathbb{Z}_{p_{k}}$-module of rank $\boldsymbol{d}_{\boldsymbol{k}}-\mathbf{1}$, and, moreover, the natural inclusion $M \hookrightarrow\left(k^{\times}\right)^{\wedge}$ is a split injection.

Proof. First, we verify assertion (i). Write $\left(k^{\times}\right)^{\wedge}\left(p_{k}\right),\left(\left(k^{(d=1)}\right)^{\times}\right)^{\wedge}\left(p_{k}\right)$ for the [uniquely determined] pro- $p_{k}$-Sylow subgroups of the abelian profinite groups $\left(k^{\times}\right)^{\wedge},\left(\left(k^{(d=1)}\right)^{\times}\right)^{\wedge}$, respectively. Let us observe that it follows immediately from [3], Lemma 1.2, (i), that, to verify assertion (i), it suffices to verify that the image $\operatorname{Nm}_{k / k^{(d=1)}}^{\wedge}\left(\left(k^{\times}\right)^{\wedge}\left(p_{k}\right)\right) \subseteq\left(\left(k^{(d=1)}\right)^{\times}\right)^{\wedge}\left(p_{k}\right)$ is torsion-free. Thus, if $p_{k}$ is $o d d$, then since [one verifies easily from Proposition 1.1, (v), that] $\left(\left(k^{(d=1)}\right)^{\times}\right)^{\wedge}\left(p_{k}\right)$ is torsion-free, assertion (i) holds.

Suppose that $p_{k}=2$. Then let us observe that since $\left(\left(k^{(d=1)}\right)^{\times}\right)_{\text {tor }}=\{ \pm 1\}$ [cf. Proposition 1.1, (v); [3], Lemma 1.2, (i)], it follows immediately from Lemma 4.1, (iii), that, to verify assertion (i) in the case where $p_{k}=2$, it suffices to verify that the image of the inertia subgroup $I_{k} \subseteq G_{k}$ by $\chi_{p_{k} \text {-cyc }}: G_{k} \rightarrow \mathbb{Z}_{p_{k}}^{\times}$ [cf. Lemma 4.1, (i)] does not contain $-1 \in \mathbb{Z}_{p_{k}}^{\times}$. On the other hand, since [we 
have assumed that] $\left(p_{k}, a_{k}\right) \neq(2,1)$, this follows immediately from the [easily verified] injectivity of the composite $\{ \pm 1\} \hookrightarrow \mathbb{Z}_{p_{k}}^{\times} \rightarrow(\mathbb{Z} / 4 \mathbb{Z})^{\times}$. This completes the proof of assertion (i) in the case where $p_{k}=2$, hence also of assertion (i).

Assertion (ii) follows immediately from assertion (i), together with [3], Lemma 1.2, (i). This completes the proof of Lemma 7.3.

Lemma 7.4. Suppose that $\left(p_{k}, a_{k}\right) \neq(2,1)$. Then there exists a Galois extension $k_{\infty} \subseteq \bar{k}$ of $k$ such that $\operatorname{Gal}\left(k_{\infty} / k\right)$ is a free $\mathbb{Z}_{p_{k}}$-module of rank $\boldsymbol{d}_{\boldsymbol{k}}-\mathbf{1}$, and, moreover, the [uniquely determined] maximal intermediate field of $k_{\infty} / k^{(d=1)}$ abelian over $k^{(d=1)}$ coincides with $k^{(\mathrm{ab})}$ [cf. Definition 4.2, (iii)].

Proof. Let us first observe that one verifies immediately from [3], Lemma 1.7, (1), (2), that, to verify Lemma 7.4, it suffices to verify that there exists a surjection $\phi:\left(k^{\times}\right)^{\wedge} \rightarrow\left(\left(\mathbb{Z}_{p_{k}}\right)_{+}\right)^{\oplus d_{k}-1}$ of profinite groups such that if we write $\operatorname{Nm}_{k / k^{(d=1)}}^{\wedge}:\left(k^{\times}\right)^{\wedge} \rightarrow\left(\left(k^{(d=1)}\right)^{\times}\right)^{\wedge}$ for the homomorphism induced by the Norm map $\mathrm{Nm}_{k / k^{(d=1)}}: k^{\times} \rightarrow\left(k^{(d=1)}\right)^{\times}$, then the equality $\operatorname{Nm}_{k / k^{(d=1)}}^{\wedge}\left(\left(k^{\times}\right)^{\wedge}\right)=$ $\mathrm{Nm}_{k / k^{(d=1)}}^{\wedge}(\operatorname{Ker}(\phi))$ holds. On the other hand, this follows immediately from Lemma 7.3, (ii). This completes the proof of Lemma 7.4.

THEOREM 7.5. Suppose that a maximal intermediate field of $k / k^{(\mathrm{ab})}$ tamely ramified over $k^{(\mathrm{ab})}$ does not coincide with $k^{(d=1)} \subseteq k$ [which is the case if, for instance, $\left.d_{k}^{(\mathrm{ab})} \neq 1\right]$, and that $\left(p_{k}, a_{k}\right) \neq(2,1)$. Let $n$ be a nonnegative integer such that $\left[k: k^{(\mathrm{ab})}\right] \in p_{k}^{n} \mathbb{Z}$ and $A$ an abelian $p_{k}$-group that satisfies the following two conditions:

(1) It holds that \#A= $p_{k}^{n}$.

(2) The finite abelian group $A$ is generated by at most $\left(d_{k} / p_{k}^{n}\right)-1$ elements.

Then there exists a subgroup of $\operatorname{Out}\left(G_{k}\right)$ isomorphic to $A$.

Proof. Let $K_{1}$ be a maximal intermediate field of $k / k^{(\mathrm{ab})}$ tamely ramified over $k^{(\mathrm{ab})}$. Thus, it follows from Lemma 4.3, (ii), that the positive integer $d_{k} / d_{K_{1}}$ is a power of $p_{k}$ and $\geq p_{k}^{n}$. Then since [we have assumed that] $d_{K_{1}} \geq 2$, it follows immediately from Lemma 7.4 that there exists a finite Galois extension $K_{2} \subseteq \bar{k}$ of $K_{1}$ such that $d_{k}=p_{k}^{n} \cdot d_{K_{2}}$ [i.e., that $d_{k} / d_{K_{1}}=p_{k}^{n} \cdot d_{K_{2}} / d_{K_{1}}$ ], and, moreover, $K_{2}^{(\mathrm{ab})}=K_{1}^{(\mathrm{ab})}\left(=k^{(\mathrm{ab})}-\mathrm{cf}\right.$. Lemma 4.3 , (i)). Thus, it follows immediately - in light of condition (2) - from Lemma 7.4 that there exists a finite Galois extension $K_{3} \subseteq \bar{k}$ of $K_{2}$ such that $\mathrm{Gal}\left(K_{3} / K_{2}\right)$ is isomorphic to $A$-which thus implies [cf. condition (1)] that $d_{K_{3}}=d_{K_{2}} \cdot \# A=d_{K_{2}} \cdot p_{k}^{n}=d_{k}$ and, moreover, $K_{3}^{(\mathrm{ab})}=K_{2}^{(\mathrm{ab})}\left(=k^{(\mathrm{ab})}\right)$. In particular, since [we have assumed that] $\left(p_{k}, a_{k}\right) \neq(2,1)$, it follows immediately - in light of Proposition 2.5, (i); Proposition 4.9, (i); [3], Proposition 3.6-from Theorem 4.11, together with [3], Lemma 1.7, (1), (2), that $G_{k}$ is isomorphic, as an abstract group, to 
$G_{K_{3}} \stackrel{\text { def }}{=} \operatorname{Gal}\left(\bar{k} / K_{3}\right)$. Thus, by considering the image of the composite of the natural injection $\operatorname{Gal}\left(K_{3} / K_{2}\right) \hookrightarrow\left(\operatorname{Aut}\left(K_{3}\right) \hookrightarrow\right) \operatorname{Out}\left(G_{K_{3}}\right)$ and the isomorphism $\operatorname{Out}\left(G_{K_{3}}\right) \stackrel{\sim}{\rightarrow} \operatorname{Out}\left(G_{k}\right)$ obtained by conjugation by some isomorphism $G_{K_{3}} \stackrel{\sim}{\rightarrow} G_{k}$, we obtain a subgroup of $\operatorname{Out}\left(G_{k}\right)$ isomorphic to $A$, as desired. This completes the proof of Theorem 7.5.

REMARK 7.5.1. One concrete application of Theorem 7.5 is as follows: Suppose that $p_{k}$ is odd. Let $n$ be a positive integer. Suppose, moreover, that $k=\mathbb{Q}_{p_{k}}\left(\zeta_{p_{k}^{n}}, p_{k}^{1 / p_{k}^{n}}\right)$. Then one verifies immediately that

$$
k^{(\mathrm{ab})}=\mathbb{Q}_{p_{k}}\left(\zeta_{p_{k}^{n}}\right), \quad d_{k}=p_{k}^{2 n-1} \cdot\left(p_{k}-1\right), \quad d_{k}^{(\mathrm{ab})}=p_{k}^{n-1} \cdot\left(p_{k}-1\right) .
$$

Thus, it follows from Theorem 7.5 that, for positive integers $d, r_{1}, \ldots, r_{d}$ such that $r_{1}+\cdots+r_{d}=n$, there exists a subgroup of the group $\operatorname{Out}\left(G_{k}\right)$ isomorphic to the abelian $p_{k}$-group

$$
\mathbb{Z} / p_{k}^{r_{1}} \mathbb{Z} \times \cdots \times \mathbb{Z} / p_{k}^{r_{d}} \mathbb{Z}
$$

[cf. the easily verified inequalities $d \leq n<d_{k} / p_{k}^{n}$ ].

Note that this observation in the case where $n=2$ was already given in example (2) given in [4], §2.

REMARK 7.5.2. One of motivations of studying Theorem 6.12, Theorem 7.2, and Theorem 7.5 is as follows:

(i) The Neukirch-Uchida theorem [cf. the main theorem of [16]] asserts that

$\left(\dagger_{\mathrm{NF}}\right)$ every outer isomorphism of profinite groups between the absolute Galois groups of number fields [i.e., finite extensions of $\mathbb{Q}$ ] arises from a uniquely determined isomorphism between the number fields,

which thus implies that

$\left(\ddagger_{\mathrm{NF}}\right)$ the isomorphism class, i.e., as an abstract profinite group, of the absolute Galois group of a number field completely determines the isomorphism class, i.e., as an abstract field, of the number field.

On the other hand, it is well-known [cf., e.g., [3], Theorem 2.2] that neither the assertion $\left(\dagger_{\mathrm{NF}}\right)$ for $M L F$ 's nor the assertion $\left(\ddagger_{\mathrm{NF}}\right)$ for $M L F$ 's holds. More precisely, for instance, if $p$ is odd, then

$\left(\dagger_{M L F}\right)$ there exists an outer automorphism of the absolute Galois group of $\mathbb{Q}_{p}$ that is nontrivial, hence also does not arise from any automorphism of the field $\mathbb{Q}_{p}$ [cf., e.g., the discussion given at the final portion of [12], Chapter VII, §5],

and, moreover,

( $\ddagger$ MLF) there exist two MLF’s $k_{\circ}, k_{\bullet}$ such that the field $k_{\circ}$ is not isomorphic to $k_{\bullet}$, but the absolute Galois group of $k_{\circ}$ is isomorphic to the absolute Galois group of $k_{\bullet}$ [cf., e.g., [17], §2, Theorem, (i)]. 
(ii) The assertion ( $\left.\dagger_{M L F}\right)$ in (i) thus asserts that, in general [e.g., in the case where we take the " $k$ " to be $\mathbb{Q}_{p}$, for some odd prime number $p$ ], the natural injection

$$
\operatorname{Aut}(k) \hookrightarrow \operatorname{Out}\left(G_{k}\right)
$$

[cf., e.g., [3], Proposition 2.1] is not bijective. Under this state of affairs, one may consider the following problem:

Problem: Is there a certain "suitable" characterization of the subgroup $\operatorname{Aut}(k) \subseteq \operatorname{Out}\left(G_{k}\right)$ of $\operatorname{Out}\left(G_{k}\right)$ ?

Here, let us observe that

Theorem 6.12, (ii), may be regarded as a certain affirmative solution to this problem, i.e., in the case where the MLF $k$ is Galois-specifiable [cf. Definition 6.1].

(iii) From the point of view of the problem in (ii), let us observe the [easily verified] finiteness of the group $\operatorname{Aut}(k)$.

In particular, as one of possible solutions to the problem in (ii), one may discuss the following question:

$\left(*_{\text {fin }}\right) \quad$ Is the subgroup $\operatorname{Aut}(k)$ of $\operatorname{Out}\left(G_{k}\right)$ the uniquely determined maximal finite subgroup of $\operatorname{Out}\left(G_{k}\right)$ ? Put another way, is every element of $\operatorname{Out}\left(G_{k}\right)$ of finite order contained in the subgroup $\operatorname{Aut}(k)$ of $\operatorname{Out}\left(G_{k}\right)$ ?

Now let us observe that it is immediate that an affirmative answer to this question $\left(*_{\text {fin }}\right)$ implies an affirmative answer to the following question $\left(*_{\mathrm{char}}\right)$, hence also an affirmative answer to the following question $\left(*_{\text {nor }}\right)$ :

$\left(*_{\text {char }}\right) \quad$ Is the subgroup $\operatorname{Aut}(k)$ of $\operatorname{Out}\left(G_{k}\right)$ characteristic?

$\left(*_{\text {nor }}\right) \quad$ Is the subgroup $\operatorname{Aut}(k)$ of $\operatorname{Out}\left(G_{k}\right)$ normal?

an affir. sol. to $\left(*_{\text {fin }}\right) \Rightarrow$ an affir. sol. to $\left(*_{\text {char }}\right) \Rightarrow$ an affir. sol. to $\left(*_{\text {nor }}\right)$

(iv) Now let us observe that

Theorem 7.2 is related to the question $\left(*_{\text {nor }}\right)$ in (iii), and that

Theorem 7.5 [cf. also the example in Remark 7.5.1] yields a negative answer to the question $\left(*_{\text {fin }}\right)$ in (iii).

(v) In $\S 8$, we will give a negative answer to the question $\left(*_{\text {nor }}\right)$ in (iii), hence also [cf. the discussion of (iii)] negative answers to the questions $\left(*_{\text {fin }}\right)$ and $\left(*_{\text {char }}\right)$ in (iii) [cf. Corollary 8.7 below]. 


\section{On outer automorphisms arising from field automorphisms II}

In the present $\S 8$, we maintain the notational conventions introduced at the beginnings of $\S 1$ and $\S 2$. In particular, we have been given a group of MLF-type

$$
G \text {. }
$$

Let $l$ be a prime number. Suppose, moreover, that

(a) $k^{\times}(G)[l] \neq\{1\}$,

(b) $d(G) / d^{(\mathrm{ab})}(G)=l[$ cf. Definition 4.7, (iv)], and, moreover,

(c) $d^{(\mathrm{ab})}(G) \notin l \mathbb{Z}$.

In the present $\S 8$, we give a negative answer to the question $\left(*_{\text {nor }}\right)$ in Remark 7.5.2, (iii) [cf. Corollary 8.7 below].

Lemma 8.1. Let $\Gamma \subseteq \operatorname{Out}(G)$ be a quasi-geometric [cf. Definition 6.5, (ii)] subgroup of order $\boldsymbol{l}$. Then the following hold:

(i) The group $G \stackrel{\text { out }}{\rtimes} \Gamma[c f$. Definition 6.5, (i)] is of AAMLF-type $[c f$. Definition 4.8] whose MLF-Galois label [cf. Definition 4.10] coincides with

$$
\left(p(G), d^{(\mathrm{ab})}(G), \operatorname{Im}\left(\mathrm{Nm}_{\mathrm{abs}}(G)\right)\right)
$$

[cf. Definition 4.7, (iii)].

(ii) The isomorphism class of the group $G \rtimes \Gamma$ out $\Gamma$ does not depend on the choice of $\Gamma$, i.e., depends only on $(G, l)$.

(iii) It holds that $e(G \stackrel{\text { out }}{\rtimes} \Gamma)=e(G) / l$, and that $k^{\times}(G \stackrel{\text { out }}{\rtimes} \Gamma)[l] \neq\{1\}$.

(iv) There exists a uniquely determined $\mathbb{F}_{l}^{\times}$-torsor

$$
T \subseteq k^{\times}(G \stackrel{\text { out }}{\rtimes} \Gamma) \otimes_{\mathbb{Z}} \mathbb{F}_{l}
$$

in the vector space $k^{\times}(G \stackrel{\text { out }}{\rtimes} \Gamma) \otimes_{\mathbb{Z}} \mathbb{F}_{l}$ over $\mathbb{F}_{l}$ that satisfies the following condition: Write $S \subseteq \bar{k}^{\times}(G)$ for the subset of $\bar{k}^{\times}(G)$ consisting of elements $\gamma \in \bar{k}^{\times}(G)\left(\supseteq \bar{k}^{\times}(G)^{G \rtimes \Gamma}=k^{\times}(G \stackrel{\text { out }}{\rtimes} \Gamma)-c f\right.$. [3], Proposition 4.2, (i)) of $\bar{k}^{\times}(G)$

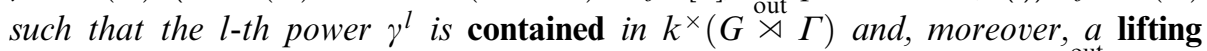
of an element of $T$, i.e., relative to the natural surjection $k^{\times}(G \stackrel{\text { out }}{\rtimes} \Gamma) \rightarrow$ $k^{\times}(G \stackrel{\text { out }}{\rtimes} \Gamma) \otimes_{\mathbb{Z}} \mathbb{F}_{l}$. Then the subset $S$ is nonempty, and, oreover, for every element $\gamma \in S$ of $S$, the subgroup $G \subseteq G \stackrel{\text { out }}{\rtimes} \Gamma$ of $G \stackrel{\text { out }}{\rtimes} \Gamma$ coincides with the stabilizer, with respect to the natural action of $G \stackrel{\text { out }}{\rtimes}$ on $\bar{k}^{\times}(G)$, of $\gamma \in S$.

Proof. First, we verify assertion (i). Let us first observe that it follows from [3], Proposition 3.6, that $p(G \stackrel{\text { out }}{\rtimes} \Gamma)=p(G)$ and $d(G \stackrel{\text { out }}{\rtimes} \Gamma)=d(G) / l=$ $d^{(\mathrm{ab})}(G)$ [cf. the condition (b) at the beginning of the present $\S 8$ ]. Thus, to verify assertion (i), it suffices to verify that $\operatorname{Im}\left(\mathrm{Nm}_{\mathrm{abs}}(G)\right)=\operatorname{Im}\left(\mathrm{Nm}_{\mathrm{abs}}(G \rtimes\right.$ $\Gamma)$ ). To this end, let us observe that it follows from Remark 4.9.1 that 
$\operatorname{Im}\left(\operatorname{Nm}_{\mathrm{abs}}(G)\right) \subseteq \operatorname{Im}\left(\mathrm{Nm}_{\mathrm{abs}}(G \stackrel{\text { out }}{\rtimes} \Gamma)\right), \quad$ and, moreover, $\quad\left[\operatorname{Im}\left(\mathrm{Nm}_{\mathrm{abs}}(G \stackrel{\text { out }}{\rtimes} \Gamma)\right)\right.$ : $\left.\operatorname{Im}\left(\operatorname{Nm}_{\mathrm{abs}}(G)\right)\right] \in\{1, l\}$. Thus, it follows from the condition $(\mathrm{c})$ at the beginning of the present $\S 8$ that $\operatorname{Im}\left(\mathrm{Nm}_{\text {abs }}(G)\right)=\operatorname{Im}\left(\mathrm{Nm}_{\text {abs }}(G \stackrel{\text { out }}{\rtimes} \Gamma)\right)$, as desired. This completes the proof of assertion (i).

Assertion (ii) follows from assertion (i) and Theorem 4.11. Next, we verify assertion (iii). The first assertion of assertion (iii) follows immediatelyin light of Proposition 4.9, (iii); [3], Proposition 3.6-from assertion (i) and Lemma 4.3, (ii). Next, let us observe that it follows immediately from [3], Proposition 4.2, (i), that, to verify the second assertion of assertion (iii), it suffices to verify that the homomorphism $G \stackrel{\text { out }}{\rtimes} \rightarrow \mathbb{F}_{l}^{\times}$determined by the natural action of $G \stackrel{\text { out }}{\rtimes} \Gamma$ on $\bar{k}^{\times}(G)[l]$ is trivial. On the other hand, it follows from the condition (a) at the beginning of the present $\S 8$, together with [3], Proposition 4.2, (i), that the restriction to $G \subseteq G \rtimes$ out $\Gamma$ of the homomorphism $G \stackrel{\text { out }}{\rtimes} \Gamma \rightarrow \mathbb{F}_{l}^{\times}$is trivial. Thus, the desired triviality follows from our assumption that $\Gamma$ is of order $l$. This completes the proof of assertion (iii). Finally, since $\Gamma$ is of order $l$, assertion (iv) follows immediately - in light of [3], Proposition 4.2, (i) - from Kummer theory, together with assertion (iii). This completes the proof of Lemma 8.1.

REMARK 8.1.1. Suppose that $p_{k}$ is odd. Let

$$
a \in \mathbb{Q}_{p_{k}}^{\times} \backslash\left(\mathbb{Q}_{p_{k}}^{\times}\right)^{p_{k}} \text {. }
$$

Suppose, moreover, that

$$
k=\mathbb{Q}_{p_{k}}\left(\zeta_{p_{k}}, a^{1 / p_{k}}\right) .
$$

Then, by the easily verified equality $\left(\mathbb{Q}_{p_{k}}^{\times}\right)^{p_{k}}=\mathbb{Q}_{p_{k}}^{\times} \cap\left(\mathbb{Q}_{p_{k}}\left(\zeta_{p_{k}}\right)^{\times}\right)^{p_{k}}$, one verifies immediately that

$$
k^{(\mathrm{ab})}=\mathbb{Q}_{p_{k}}\left(\zeta_{p_{k}}\right), \quad d_{k}=p_{k} \cdot\left(p_{k}-1\right), \quad d_{k}^{(\mathrm{ab})}=p_{k}-1
$$

[cf. Definition 4.2, (iii), (iv)]. Thus, it follows from Proposition 4.9, (ii); [3], Proposition 3.6; [3], Proposition 3.11, (i), that the group $G_{k}$ of MLF-type satisfies the three conditions (a), (b), and (c) at the beginning of the present $\S 8$ in the case where we take the prime number " $l$ " to be $p_{k}$. Moreover, in this case, by Lemma 6.6, (i), and Proposition 6.7, the subgroup

$$
\operatorname{Gal}\left(k / \mathbb{Q}_{p_{k}}\left(\zeta_{p_{k}}\right)\right) \subseteq\left(\operatorname{Aut}(k)=\operatorname{Gal}\left(k / \mathbb{Q}_{p_{k}}\right) \subseteq\right) \operatorname{Out}\left(G_{k}\right)
$$

yields an example of a quasi-geometric subgroup of $\operatorname{Out}\left(G_{k}\right)$ of order $p_{k}$, i.e., as discussed in Lemma 8.1.

Definition 8.2. Let $\Gamma \subseteq \operatorname{Out}(G)$ be a quasi-geometric subgroup of order $l$. 
(i) We shall write

$$
T(\Gamma) \subseteq k^{\times}(G \stackrel{\text { out }}{\rtimes} \Gamma) \otimes_{\mathbb{Z}} \mathbb{F}_{l}
$$

for the uniquely determined $\mathbb{F}_{l}^{\times}$-torsor " $T$ " of Lemma 8.1, (iv).

(ii) We shall refer to an element of the subset " $S$ " of Lemma 8.1 , (iv), as a Kummer generator for $\Gamma$. Note that it follows from Lemma 8.1, (iv), that every Kummer generator for $\Gamma$ is contained in $k^{\times}(G)\left(=\bar{k}^{\times}(G)^{G} \subseteq\right.$ $\left.\bar{k}^{\times}(G)\right)$.

(iii) We shall say that $\Gamma$ is of unit-Kummer type if the image of the $\mathbb{F}_{l}^{\times}$-torsor $T(\Gamma) \subseteq k^{\times}(G \stackrel{\text { out }}{\rtimes} \Gamma) \otimes_{\mathbb{Z}} \mathbb{F}_{l}$ of (i) by the homomorphism

$$
k^{\times}(G \stackrel{\text { out }}{\rtimes} \Gamma) \otimes_{\mathbb{Z}} \mathbb{F}_{l} \rightarrow\left(\mathbb{F}_{l}\right)_{+}
$$

induced by $\operatorname{ord}_{凶}(G \stackrel{\text { out }}{\rtimes} \Gamma)$ [cf. Definition 2.2] is $\{0\}$ [or, alternatively, $\left.\neq\left(\mathbb{F}_{l}\right)_{+} \backslash\{0\}\right]$.

(iv) Let $\Gamma_{\text {st }} \subseteq \operatorname{Out}(G)$ be a strictly quasi-geometric [cf. Definition 6.5, (iii)] subgroup that contains $\Gamma$. Then we shall say that $\Gamma$ is of $\Gamma_{\mathrm{st}}$-Kummer type if there exists a Kummer generator $\gamma \in \bar{k}^{\times}(G)$ for $\Gamma_{\text {out }}$ such that the $l$-th power $\gamma^{l} \in \bar{k}^{\times}(G)$ is contained in $k^{\times}\left(G \stackrel{\text { out }}{\rtimes} \Gamma_{\text {st }}\right)\left(\subseteq k^{\times}(G \stackrel{\text { out }}{\rtimes} \Gamma)\right)$.

Remark 8.2.1. One verifies immediately from [3], Proposition 4.2, (i), together with the various definitions involved, that, in the situation of Definition 8.2, (iv), the following two conditions are equivalent:

(1) The quasi-geometric subgroup $\Gamma$ is of $\Gamma_{\mathrm{st}}$-Kummer type.

(2) There exists an element $a \in k\left(G \rtimes{ }^{\text {out }} \Gamma_{\text {st }}\right)$ of the $\operatorname{MLF} k\left(G \stackrel{\text { out }}{\rtimes} \Gamma_{\text {st }}\right)$ [cf. Definition 5.2, Remark 5.2.1] such that the subgroup $G \subseteq G \stackrel{\text { out }}{\rtimes} \Gamma_{\text {st }}$ of $G \stackrel{\text { out }}{\rtimes} \Gamma_{\text {st }}$ coincides with the intersection

$$
(G \stackrel{\text { out }}{\rtimes} \Gamma) \cap\left(G \stackrel{\text { out }}{\rtimes} \Gamma_{\text {st }}\right)(l ; 1 ; l ; a)
$$

of the subgroup $G \stackrel{\text { out }}{\rtimes} \Gamma \subseteq G \stackrel{\text { out }}{\rtimes} \Gamma_{\text {st }}$ and the $\operatorname{subgroup~}\left(G \stackrel{\text { out }}{\rtimes} \Gamma_{\text {st }}\right)(l ; 1 ; l ; a) \subseteq$ $G \stackrel{\text { out }}{\rtimes} \Gamma_{\text {st }}$ of Definition 5.8 , (ii), by the strictly radical data [cf. Definition 5.6, (i)] $(l ; 1 ; l ; a)$ for $k\left(G \stackrel{\text { out }}{\rtimes} \Gamma_{\text {st }}\right)$.

REMARK 8.2.2. Suppose that we are in the situation of Remark 8.1.1. Then one verifies immediately from the various definitions involved that the following hold:

(i) The $\mathbb{F}_{p_{k}}^{\times}$-torsor

$$
T\left(\operatorname{Gal}\left(k / \mathbb{Q}_{p_{k}}\left(\zeta_{p_{k}}\right)\right)\right) \subseteq k^{\times}\left(G_{k} \stackrel{\text { out }}{\rtimes} \operatorname{Gal}\left(k / \mathbb{Q}_{p_{k}}\left(\zeta_{p_{k}}\right)\right)\right) \otimes_{\mathbb{Z}} \mathbb{F}_{p_{k}} \simeq \mathbb{Q}_{p_{k}}\left(\zeta_{p_{k}}\right)^{\times} \otimes_{\mathbb{Z}} \mathbb{F}_{p_{k}}
$$

[cf. [3], Proposition 3.11, (i)] is given by the $\mathbb{F}_{p_{k}}^{\times}$-torsor obtained by forming the $\mathbb{F}_{p_{k}}^{\times}$-orbit of the image of $a \in \mathbb{Q}_{p_{k}}^{\times} \subseteq \mathbb{Q}_{p_{k}}\left(\zeta_{p_{k}}\right)^{\times}$in $\mathbb{Q}_{p_{k}}\left(\zeta_{p_{k}}\right)^{\times} \otimes_{\mathbb{Z}} \mathbb{F}_{p_{k}}$. 
(ii) The element $a^{1 / p_{k}} \in k^{\times} \stackrel{\sim}{\rightarrow} k^{\times}\left(G_{k}\right)$ [cf. [3], Proposition 3.11, (i)] is a Kummer generator for the quasi-geometric subgroup $\operatorname{Gal}\left(k / \mathbb{Q}_{p_{k}}\left(\zeta_{p_{k}}\right)\right)$ of order $p_{k}$.

(iii) It holds that the quasi-geometric subgroup $\operatorname{Gal}\left(k / \mathbb{Q}_{p_{k}}\left(\zeta_{p_{k}}\right)\right)$ of order $p_{k}$ is of unit-Kummer type if and only if $a \in \mathbb{Z}_{p_{k}}^{\times} \cdot\left(\mathbb{Q}_{p_{k}}^{\times}\right)^{p_{k}}$.

(iv) Let us observe that, by Proposition 6.7, the subgroup

$$
\left(\operatorname{Gal}\left(k / \mathbb{Q}_{p_{k}}\left(\zeta_{p_{k}}\right)\right) \subseteq\right) \operatorname{Aut}(k)=\operatorname{Gal}\left(k / \mathbb{Q}_{p_{k}}\right) \subseteq \operatorname{Out}\left(G_{k}\right)
$$

yields an example of a strictly quasi-geometric subgroup of $\operatorname{Out}\left(G_{k}\right)$ that contains the quasi-geometric subgroup $\operatorname{Gal}\left(k / \mathbb{Q}_{p_{k}}\left(\zeta_{p_{k}}\right)\right)$ of order $p_{k}$, i.e., as discussed in Definition 8.2, (iv). Moreover, in this case, since [we have assumed that] $a \in \mathbb{Q}_{p_{k}}^{\times}\left(=\left(k^{\times}\right)^{\operatorname{Aut}(k)}\right)$, the quasi-geometric subgroup $\operatorname{Gal}\left(k / \mathbb{Q}_{p_{k}}\left(\zeta_{p_{k}}\right)\right)$ of order $p_{k}$ is of $\operatorname{Aut}(k)$-Kummer type.

Lemma 8.3. Let $\Gamma, \Sigma \subseteq \operatorname{Out}(G)$ be quasi-geometric subgroups of order $\boldsymbol{l}$. Suppose that $\Gamma$ is not of unit-Kummer type. Let $\gamma \in k^{\times}(G)$ be a Kummer generator for $\Gamma$. Then the following hold:

(i) It holds that $\gamma \notin k^{\times}(G \stackrel{\text { out }}{\rtimes} \Sigma)$.

(ii) Suppose that $\gamma^{l} \in k^{\times}(G \underset{\text { out }}{\rtimes} \Sigma)$. Then $\gamma$ is a Kummer generator for $\Sigma$.

(iii) Suppose that $\gamma^{l} \in k^{\times}(G \stackrel{\text { out }}{\rtimes} \Sigma)$. Then the quasi-geometric subgroup $\Sigma$ is not of unit-Kummer type.

Proof. First, we verify assertion (i). Let us first observe that since $\Gamma$ is of order $l$ and not of unit-Kummer type, it follows immediately from Proposition 2.3 that $\operatorname{ord}_{凶}(G)(\gamma) \notin l \mathbb{Z}$. On the other hand, since $\Sigma$ is of order $l$, it follows immediately from Proposition 2.3 and Lemma 8.1, (iii), that $\operatorname{ord}_{\bigotimes}(G)\left(k^{\times}(G \stackrel{\text { out }}{\rtimes} \Sigma)\right)=l \mathbb{Z}$. Thus, assertion (i) holds. This completes the proof of assertion (i).

Next, since $k^{\times}(G \stackrel{\text { out }}{\rtimes} \Sigma)[l] \neq\{1\}$ [cf. Lemma 8.1, (iii)], and $\Sigma$ is of order l, assertion (ii) follows immediately-in light of [3], Proposition 4.2, (i)-from assertion (i) and Kummer theory. Finally, since $\operatorname{ord}_{\square}(G)(\gamma) \notin l \mathbb{Z}$ [cf. the proof of assertion (i)], and $\Sigma$ is of order l, assertion (iii) follows immediately - in light of Proposition 2.3-from assertion (ii) and Lemma 8.1, (iii). This completes the proof of Lemma 8.3.

LEMma 8.4. Let $\Gamma_{\mathrm{st}} \subseteq \operatorname{Out}(G)$ be a strictly quasi-geometric subgroup of $\operatorname{Out}(G)$. Then the following hold:

(i) The group $\Gamma_{\text {st }}$ has a uniquely determined -Sylow subgroup. Moreover, the l-Sylow subgroup is of order $\boldsymbol{l}$.

(ii) Let $\Sigma \subseteq \operatorname{Out}(G)$ be a subgroup of $\operatorname{Out}(G)$ such that $\Sigma \subseteq N_{\operatorname{Out}(G)}\left(\Gamma_{\mathrm{st}}\right)$. Then it holds that

$$
k^{\times}\left(G \stackrel{\text { out }}{\rtimes} \Gamma_{\text {st }}\right) \subseteq k^{\times}(G)^{\Sigma}
$$


(iii) In the situation of (ii), suppose, moreover, that $\Sigma$ is quasi-geometric. Then it holds that

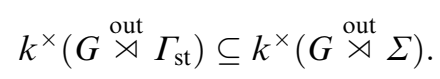

Proof. First, we verify assertion (i). Let us first observe that it follows from the conditions (b), (c) at the beginning of the present $\S 8$, together with Lemma 6.6, (iv), that each $l$-Sylow subgroup of $\Gamma_{\text {st }}$ is of order $l$. Let $\Gamma_{1}$, $\Gamma_{2} \subseteq \Gamma_{\text {st }}$ be $l$-Sylow subgroups of $\Gamma_{\text {st }}$ [which thus implies that $\left.\# \Gamma_{1}=\# \Gamma_{2}=l\right]$. Then since both $\Gamma_{1}$ and $\Gamma_{2}$ are quasi-geometric [cf. Lemma 6.6, (i)], it follows immediately from Lemma 8.1, (i), that $G \stackrel{\text { out }}{\rtimes} \Gamma_{1}=G \stackrel{\text { out }}{\rtimes} \Gamma_{2}$, i.e., as subgroups of $G \stackrel{\text { out }}{\rtimes} \Gamma_{\text {st }}$. In particular, we obtain that $\Gamma_{1}=\Gamma_{2}$, i.e., as subgroups of $\operatorname{Out}(G)$, as desired. This completes the proof of assertion (i).

Next, since $\Gamma_{\mathrm{st}}$ is strictly quasi-geometric [which thus implies that $\left.d\left(G \stackrel{\text { out }}{\rtimes} \Gamma_{\text {st }}\right)=1\right]$, assertion (ii) follows immediately from Corollary 5.5. Finally, assertion (iii) follows from assertion (ii), together with Lemma 6.6, (ii). This completes the proof of Lemma 8.4.

THEOREM 8.5. Let $\Gamma_{\text {st }} \subseteq \operatorname{Out}(G)$ be a strictly quasi-geometric subgroup of $\operatorname{Out}(G)$ and $\Sigma \subseteq \operatorname{Out}(G)$ a quasi-geometric subgroup of order $\boldsymbol{l}$. Write $\Gamma \subseteq \Gamma_{\text {st }}$ for the uniquely determined l-Sylow subgroup of $\Gamma_{\mathrm{st}}[c f$. Lemma 8.4, (i)]. Suppose that the following three conditions are satisfied:

(1) The subgroup $\Sigma \subseteq \operatorname{Out}(G)$ normalizes the subgroup $\Gamma_{\mathrm{st}} \subseteq \operatorname{Out}(G)$.

(2) The quasi-geometric subgroup $\Gamma$ is of $\Gamma_{\mathrm{st}}$-Kummer type.

(3) The quasi-geometric subgroup $\Gamma$ is not of unit-Kummer type.

Then the quasi-geometric subgroup $\Sigma$ is not of unit-Kummer type.

Proof. It follows from Lemma 8.4, (iii), and condition (1) that $k^{\times}(G \stackrel{\text { out }}{\rtimes}$

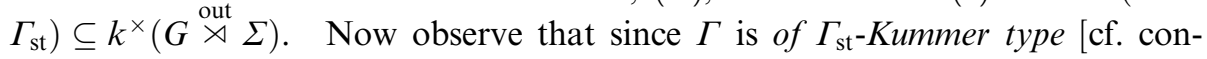
dition (2)], there exists a Kummer generator $\gamma \in k^{\times}(G)$ for $\Gamma$ such that $\gamma^{l} \in$ $k^{\times}\left(G \stackrel{\text { out }}{\rtimes} \Gamma_{\text {st }}\right)$, which thus implies [cf. the above inclusion $k^{\times}\left(G \stackrel{\text { out }}{\rtimes} \Gamma_{\text {st }}\right) \subseteq$ $\left.k^{\times}(G \stackrel{\text { out }}{\rtimes} \Sigma)\right]$ that $\gamma^{l} \in k^{\times}(G \stackrel{\text { out }}{\rtimes} \Sigma)$. Thus, since $\Gamma$ is not of unit-Kummer type [cf. condition (3)], it follows from Lemma 8.3, (iii), that $\Sigma$ is not of unitKummer type, as desired. This completes the proof of Theorem 8.5.

Corollary 8.6. Suppose that $p_{k}$ is odd. For each $\square \in\{0, \bullet\}$, let

$$
a_{\square} \in \mathbb{Q}_{p_{k}}^{\times} \backslash\left(\mathbb{Q}_{p_{k}}^{\times}\right)^{p_{k}} ;
$$

write

$$
k_{\square} \stackrel{\text { def }}{=} \mathbb{Q}_{p_{k}}\left(\zeta_{p_{k}}, a_{\square}^{1 / p_{k}}\right), \quad G_{\square} \stackrel{\text { def }}{=} \operatorname{Gal}\left(\bar{k}_{\square} / k_{\square}\right)
$$

—where $\bar{k}_{\square}$ is an algebraic closure of $k_{\square} . \quad$ [So it follows from Theorem 4.11, together with Remark 8.1.1—cf. also Proposition 4.9, (i); [3], Proposition 3.6- 
that the group $G_{\circ}$ is isomorphic, as an abstract group, to $G_{\bullet}$.] Write

$$
\Phi: \operatorname{Out}\left(G_{\circ}\right) \stackrel{\sim}{\rightarrow} \operatorname{Out}\left(G_{\bullet}\right)
$$

for the isomorphism obtained by conjugation by some fixed isomorphism $G_{\circ} \stackrel{\sim}{\rightarrow} G_{\bullet}$ of groups. Suppose that $a_{\circ} \in \mathbb{Z}_{p_{k}}^{\times}\left[\right.$e.g., $\left.a_{\circ}=p_{k}+1\right]$ but $a_{\bullet} \notin \mathbb{Z}_{p_{k}}^{\times}\left[\right.$e.g., $\left.a_{\bullet}=p_{k}\right]$. Then it holds that

$$
\Phi\left(\operatorname{Gal}\left(k_{\circ} / \mathbb{Q}_{p_{k}}\left(\zeta_{p_{k}}\right)\right)\right) \nsubseteq N_{\operatorname{Out}\left(G_{\bullet}\right)}\left(\operatorname{Aut}\left(k_{\bullet}\right)\right) .
$$

Proof. This assertion follows immediately from Theorem 8.5, together with Remark 8.1.1 and Remark 8.2.2, (iii), (iv).

REMARK 8.6.1.

(i) Let us recall from Theorem 6.12, (i), that if $k$ is Galois-specifiable [cf. Definition 6.1], then there is a precisely one $\operatorname{Out}\left(G_{k}\right)$-conjugacy class of strictly quasi-geometric subgroups of $\operatorname{Out}\left(G_{k}\right)$.

(ii) Next, suppose that we are in the situation of Corollary 8.6. Then it follows immediately from Corollary 8.6 that $\Phi\left(\operatorname{Aut}\left(k_{\circ}\right)\right) \neq \operatorname{Aut}\left(k_{\bullet}\right)$. On the other hand, it follows from Proposition 6.7 that both $\Phi\left(\operatorname{Aut}\left(k_{\circ}\right)\right)$ and $\operatorname{Aut}\left(k_{\bullet}\right)$ are strictly quasi-geometric. Thus, [since one may take the isomorphism " $G_{\circ} \stackrel{\sim}{\rightarrow} G_{\bullet}$ " of Corollary 8.6 to be an arbitrary isomorphism] there are at least two $\operatorname{Out}\left(G_{\bullet}\right)$-conjugacy classes of strictly quasi-geometric subgroups of $\operatorname{Out}\left(G_{\bullet}\right)$. In particular - in light of Theorem 5.9, (ii)-we conclude that, in general, a similar assertion to Theorem 6.12, (i), for [the absolute Galois group of] an absolutely characteristic [cf. Definition 5.7] MLF does not hold.

COROLlary 8.7. Suppose that $p_{k}$ is odd, and that

$$
k=\mathbb{Q}_{p_{k}}\left(\zeta_{p_{k}}, p_{k}^{1 / p_{k}}\right) \text {. }
$$

Then the subgroup

$$
\operatorname{Aut}(k) \subseteq \operatorname{Out}\left(G_{k}\right)
$$

is neither normally terminal nor normal.

Proof. Since $k$ is absolutely strictly radical [cf. Definition 5.6, (iii)], hence also absolutely characteristic [cf. Theorem 5.9, (ii)], it follows from Theorem 7.2, (i), that the subgroup $\operatorname{Aut}(k) \subseteq \operatorname{Out}\left(G_{k}\right)$ of $\operatorname{Out}\left(G_{k}\right)$ is not normally terminal. Moreover, it follows immediately from Corollary 8.6 that the subgroup $\operatorname{Aut}(k) \subseteq \operatorname{Out}\left(G_{k}\right)$ of $\operatorname{Out}\left(G_{k}\right)$ is not normal. This completes the proof of Corollary 8.7 .

REMARK 8.7.1. In the present Remark 8.7.1, let us recall some of the discussions of the present $\S 8$ from the point of view of the notion of "link" [cf. [9], §2.7, (i)] as follows: 
(i) Let us apply the notational conventions introduced in the statement of Corollary 8.6. In particular, the prime number $p_{k}$ is odd. Moreover, for each $\square \in\{\circ, \bullet\}$, we are given an element

$$
a_{\square} \in \mathbb{Q}_{p_{k}}^{\times} \backslash\left(\mathbb{Q}_{p_{k}}^{\times}\right)^{p_{k}},
$$

an MLF

$$
k_{\square} \stackrel{\text { def }}{=} \mathbb{Q}_{p_{k}}\left(\zeta_{p_{k}}, a_{\square}^{1 / p_{k}}\right),
$$

and an algebraic closure

$$
\bar{k}_{\square}
$$

of $k_{\square}$. Here, let us recall that the MLF $k_{\square}$-that is one of the main arithmetic holomorphic objects [cf. [9], §2.7, (vii)] in this discussion-determines some mono-analytic objects [cf. [9], §2.7, (vii)]. For instance, we have

- the group of MLF-type

$$
G_{\square} \stackrel{\text { def }}{=} \operatorname{Gal}\left(\bar{k}_{\square} / k_{\square}\right)
$$

obtained by forming the absolute Galois group of $k_{\square}$ and

- the $\mathrm{MLF}^{\diamond}$-pair [cf. [3], Definition 5.3]

$$
G_{\square} \curvearrowright \bar{k}_{\square}^{\times}
$$

obtained by considering the natural action of $G_{\square}$ on $\bar{k}_{\square}^{\times}$.

In the remainder of Remark 8.7.1, suppose that we are in a situation in which

we are interested in a certain "characterization" of the element $a_{\square} \in \mathbb{Q}_{p_{k}}^{\times}$from the point of view of such mono-analytic objects associated to the arithmetic holomorphic object $k_{\square}$.

More specifically, suppose that we are in a situation in which

we are interested in a certain "comparison" between $a_{\circ}$ and $a_{\bullet}$ via a suitable "link" that relates such mono-analytic objects associated to $k_{\circ}$ and $k_{\bullet}$.

a "link", i.e., a suitable isomorphism

mono-an. obj. of $k_{\circ} \underset{?}{\stackrel{\sim}{\rightarrow}}$ mono-an. obj. of $k_{\bullet}$

$$
\stackrel{?}{\Rightarrow} \quad \text { a "comparison" betw. } a_{\circ} \text { and } a_{\bullet}
$$

(ii) Let us start by observing that, for each $\square \in\{\circ, \bullet\}$, the group $G_{\square}$ of MLF-type does not give any "characterization" of the element $a_{\square} \in \mathbb{Q}_{p_{k}}^{\times}$. 
Indeed, one verifies easily that

$$
k_{\square}^{(\mathrm{ab})}=\mathbb{Q}_{p_{k}}\left(\zeta_{p_{k}}\right), \quad d_{k_{\square}}=p_{k} \cdot\left(p_{k}-1\right), \quad d_{k_{\square}}^{(\mathrm{ab})}=p_{k}-1 .
$$

Thus, it follows immediately from Theorem 4.11 [cf. also Proposition 4.9, (i); [3], Proposition 3.6] that the isomorphism class of the group " $G_{\square}$ " does not depend on the choice of " $a_{\square}$ ". In particular, we have an isomorphism of groups

$$
G_{\circ} \stackrel{\sim}{\rightarrow} G_{\bullet} .
$$

As a consequence, one may conclude that

one cannot obtain "any information about $a_{\square}$ " if one considers only " $G_{\square}$ ".

(iii) In order to obtain a certain "comparison" between $a_{\circ}$ and $a_{\bullet}$, let us relate mono-analytic portions of the arithmetic holomorphic structures of $k_{\circ}$ and $k_{\text {. }}$ as follows: In the remainder of Remark 8.7.1, let us fix an isomorphism of [abstract] groups [i.e., between Frobenius-like portions-cf. [3], Definition 5.4]

$$
\alpha_{\mathrm{Fr}}: \bar{k}_{\circ}^{\times} \stackrel{\sim}{\rightarrow} \bar{k}_{\bullet}^{\times}
$$

such that the isomorphism $\operatorname{Aut}\left(\bar{k}_{\circ}^{\times}\right) \stackrel{\sim}{\rightarrow} \operatorname{Aut}\left(\bar{k}_{\bullet}^{\times}\right)$obtained by conjugation by $\alpha_{\mathrm{Fr}}$ restricts to an isomorphism of $G_{\circ} \subseteq \operatorname{Aut}\left(\bar{k}_{\circ}^{\times}\right)$with $G_{\bullet} \subseteq \operatorname{Aut}\left(\bar{k}_{\bullet}^{\times}\right)$. Write

$$
\alpha_{\text {ét }}: G_{\circ} \stackrel{\sim}{\rightarrow} G_{\bullet}
$$

for the resulting isomorphism [i.e., between étale-like portions-cf. [3], Definition 5.4]. [So the pair $\left(\alpha_{\mathrm{Fr}}, \alpha_{\text {ét }}\right)$ determines an isomorphism

$$
\left(\alpha_{\mathrm{Fr}}, \alpha_{\mathrm{et}}\right):\left(G_{\circ} \curvearrowright \bar{k}_{\circ}^{\times}\right) \stackrel{\sim}{\rightarrow}\left(G_{\bullet} \curvearrowright \bar{k}_{\bullet}^{\times}\right)
$$

of $\mathrm{MLF}^{\diamond}$-pairs-cf. [3], Definition 5.1, (ii).] In particular, roughly speaking, we are in a situation in which the collection of data " $G_{\square} \curvearrowright \bar{k}_{\square}^{\times}$" may be regarded as a coric object [i.e., roughly speaking, an object that admits the property of being invariant with respect to the "link" under consideration-cf. [9], §2.7, (iv)] of our "link" [i.e., the pair $\left.\left(\alpha_{\mathrm{Fr}}, \alpha_{\text {ét }}\right)\right]$.

Note that we have natural inclusions of groups

$$
G_{\square} \subseteq G_{\square}^{\Gamma} \stackrel{\text { def }}{=} \operatorname{Gal}\left(\bar{k}_{\square} / k_{\square}^{(\mathrm{ab})}\right) \subseteq \operatorname{Aut}\left(\bar{k}_{\square}\right) \subseteq \operatorname{Aut}\left(\bar{k}_{\square}^{\times}\right) .
$$

Write

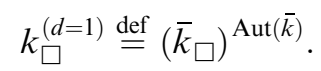

[So $k_{\square}^{(d=1)}=\mathbb{Q}_{p_{k}}$ in $\bar{k}_{\square}$.] Thus, we have a natural identification

$$
\operatorname{Aut}\left(\bar{k}_{\square}\right)=\operatorname{Gal}\left(\bar{k}_{\square} / k_{\square}^{(d=1)}\right) .
$$


In particular, the group $\operatorname{Aut}\left(\bar{k}_{\square}\right)$ is a group of $M L F$-type such that $d\left(\operatorname{Aut}\left(\bar{k}_{\square}\right)\right)$ $=1$ [cf. [3], Proposition 3.6].

(iv) Before proceeding, we pause to recall some of the discussions of $\S 5$ from the point of view of this situation. Let us recall that the reconstruction algorithms of Definition 5.2 and Definition 5.8, (i), assert that, in this situation,

(*): the MLF $k_{\square}^{(d=1)}$, as well as the collection of strictly radical data for the MLF $k_{\square}^{(d=1)}$ [cf. Definition 5.6, (i)] each member of which yields the absolutely strictly radical MLF $k_{\square}$ [e.g., the strictly radical data $\left(p_{k} ; 1 ; p_{k} ; a_{\square}\right)$ for $\left.k_{\square}^{(d=1)}\right]$, is "intrinsic" from the point of view of the collection of data

$$
G_{\square} \hookrightarrow \operatorname{Aut}\left(\bar{k}_{\square}\right) \curvearrowright \bar{k}_{\square}^{\times}
$$

-i.e., the $\mathrm{MLF}^{\diamond}$-pair $\operatorname{Aut}\left(\bar{k}_{\square}\right) \curvearrowright \bar{k}_{\square}^{\times}$equipped with the subgroup $G_{\square} \subseteq \operatorname{Aut}\left(\bar{k}_{\square}\right)$ of the étale-like portion $\operatorname{Aut}\left(\bar{k}_{\square}\right)$.

Here, suppose that the "link" of (iii) [i.e., the pair $\left.\left(\alpha_{\mathrm{Fr}}, \alpha_{\text {ét }}\right)\right]$ satisfies the condition that

$\left(\dagger_{1}\right)$ : the isomorphism $\operatorname{Aut}\left(\bar{k}_{\circ}^{\times}\right) \stackrel{\sim}{\rightarrow} \operatorname{Aut}\left(\bar{k}_{\bullet}^{\times}\right)$obtained by conjugation by $\alpha_{\mathrm{Fr}}$ also restricts to an isomorphism of $\operatorname{Aut}\left(\bar{k}_{\circ}\right) \subseteq \operatorname{Aut}\left(\bar{k}_{\circ}^{\times}\right)$with $\operatorname{Aut}\left(\bar{k}_{\bullet}\right) \subseteq \operatorname{Aut}\left(\bar{k}_{\bullet}^{\times}\right)$.

[Put another way, roughly speaking, we are in a situation in which the collection of data "Aut $\left(\bar{k}_{\square}\right) \curvearrowright \bar{k}_{\square}^{\times}$" may be regarded as a coric object of our "link".] Then we conclude immediately from the above $(*)$ that

the $\left(\left(k_{\circ}^{(d=1)}\right)^{\times}\right)^{p_{k}}$-orbit of $a_{\circ} \in\left(k_{\circ}^{(d=1)}\right)^{\times}$coincides-relative to some isomorphism $k_{\circ}^{(d=1)} \stackrel{\sim}{\rightarrow} k_{\bullet}^{(d=1)}$ of fields [i.e., determined by either the restriction $\left.\alpha_{\mathrm{Fr}}\right|_{\left(k_{\circ}^{(d=1)}\right)^{\times}}$or the composite of $\left.\alpha_{\mathrm{Fr}}\right|_{\left(k_{\circ}^{(d=1)}\right)^{\times}}$and the auto-

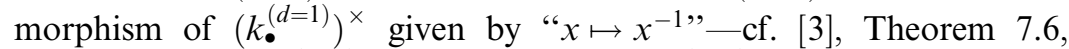
(ii)] - with the $\left(\left(k_{\bullet}^{(d=1)}\right)^{\times}\right)^{p_{k}}$-orbit of $a_{\bullet} \in\left(k_{\bullet}^{(d=1)}\right)^{\times}$,

i.e., obtain a certain "comparison" between $a_{\circ}$ and $a_{\bullet}$. As a result,

the field $k_{\circ}$ is isomorphic, as an abstract field, to the field $k_{\bullet}$.

This is precisely what is achieved by the application of the "tautological" assertion in Remark 5.9.3, (i), to absolutely strictly radical MLF's [cf. Remark 5.9.3, (ii)].

Put another way, roughly speaking,

in the situation of (iv), one may characterize the element $a_{\square}$ up to the indeterminacies arise from the action of the group $\left(\left(k_{\square}^{(d=1)}\right)^{\times}\right)^{p_{k}}$. 


$$
\begin{aligned}
& \operatorname{Aut}\left(\bar{k}_{\circ}\right) \stackrel{\sim}{\rightarrow} \operatorname{Aut}\left(\bar{k}_{\bullet}\right) \\
& \stackrel{\curvearrowright}{\bar{k}_{\circ}^{\times}} \stackrel{\sim}{\rightarrow} \quad \bar{k}_{\bullet}^{\times} \quad \Rightarrow a_{\circ} \mapsto a_{\bullet} \text { up to }\left(\left(k_{\circ}^{(d=1)}\right)^{\times}\right)^{p_{k}} \stackrel{\sim}{\rightarrow}\left(\left(k_{\bullet}^{(d=1)}\right)^{\times}\right)^{p_{k}}
\end{aligned}
$$

(v) Let us return to the situation of the present $\S 8$. Next, suppose that the "link" of (iii) [i.e., the pair $\left.\left(\alpha_{\mathrm{Fr}}, \alpha_{\mathrm{e} t}\right)\right]$ satisfies the condition that

$\left(\dagger_{2}\right)$ : the action of the subgroup $G_{\circ}^{\Gamma} \subseteq \operatorname{Aut}\left(\bar{k}_{\circ}^{\times}\right)$on $\operatorname{Aut}\left(\bar{k}_{\bullet}^{\times}\right)$by conjugation-relative to the isomorphism $\operatorname{Aut}\left(\bar{k}_{\circ}^{\times}\right) \stackrel{\sim}{\rightarrow} \operatorname{Aut}\left(\bar{k}_{\bullet}^{\times}\right)$by $\alpha_{\mathrm{Fr}}$-preserves the subgroup $\operatorname{Aut}\left(\bar{k}_{\bullet}\right) \subseteq \operatorname{Aut}\left(\bar{k}_{\bullet}^{\times}\right)$.

[Note that one verifies easily that the condition $\left(\dagger_{1}\right)$ in (iv) implies this condition $\left(\dagger_{2}\right)$.]

In this situation, we are not given any isomorphism of $\operatorname{Aut}\left(\bar{k}_{\circ}\right)$ with $\operatorname{Aut}\left(\bar{k}_{\bullet}\right)$. [Put another way, roughly speaking, we cannot regard the collection of data "Aut $\left(\bar{k}_{\square}\right) \curvearrowright \bar{k}_{\square}^{\times}$" as a coric object of our "link".] In particular, we cannot apply the reconstruction algorithm of Definition 5.8, (i). Nevertheless, Theorem 8.5 allows one to conclude that

if the $\left(\left(k_{\circ}^{(d=1)}\right)^{\times}\right)^{p_{k}}$-orbit of $a_{\circ} \in\left(k_{\circ}^{(d=1)}\right)^{\times}$contains a unit of $\mathcal{O}_{k_{\circ}^{(d=1)}}$, then the $\left(\left(k_{\bullet}^{(d=1)}\right)^{\times}\right)^{p_{k}}$-orbit of $a_{\bullet} \in\left(k_{\bullet}^{(d=1)}\right)^{\times}$contains a unit of $\mathcal{O}_{k_{\bullet}^{(d=1)}}$,

which thus implies that

$$
\text { if } \operatorname{ord}_{k_{\circ}^{(d=1)}}\left(a_{\circ}\right)=0 \text {, then } \operatorname{ord}_{k_{\bullet}^{(d=1)}}\left(a_{\bullet}\right) \in p_{k} \mathbb{Z} .
$$

In particular, we obtain a certain "comparison" between $a_{\circ}$ and $a_{\bullet}$.

$$
\begin{array}{rlrl}
G_{\circ}^{\Gamma} \supseteq G_{\circ} & \stackrel{\sim}{\rightarrow} G_{\bullet} \subseteq \operatorname{Aut}\left(\bar{k}_{\bullet}\right) \\
\curvearrowright & \stackrel{\curvearrowright}{\curvearrowright} & \\
\bar{k}_{\circ}^{\times} & \stackrel{\sim}{\rightarrow} & \bar{k}_{\bullet}^{\times}, & G_{\circ}^{\Gamma} \curvearrowright \bar{k}_{\bullet}^{\times} \operatorname{preserves} \operatorname{Aut}\left(\bar{k}_{\bullet}\right)
\end{array}
$$

Then: $\operatorname{ord}_{k_{\circ}^{(d=1)}}\left(a_{\circ}\right)=0 \Rightarrow \operatorname{ord}_{k_{\bullet}^{(d=1)}}\left(a_{\bullet}\right) \in p_{k} \mathbb{Z}$

(vi) Finally, in order to obtain an application of the conclusion of the discussion of $(\mathrm{v})$, let us take the " $\left(a_{\circ}, a_{\bullet}\right)$ " to be $\left(p_{k}+1, p_{k}\right)$. Then it follows from the discussion of (ii) that the group $G_{\circ}$ is isomorphic to the group $G_{\bullet}$. Thus, by applying a technique of mono-anabelian transport [cf. [3], Theorem 7.6, (i); also [3], Remark 7.6.1, (i)], we obtain a "link"

$$
\left(\alpha_{\mathrm{Fr}}, \alpha_{\mathrm{et}}\right):\left(G_{\circ} \curvearrowright \bar{k}_{\circ}^{\times}\right) \stackrel{\sim}{\rightarrow}\left(G_{\bullet} \curvearrowright \bar{k}_{\bullet}^{\times}\right)
$$

as in (iii). In particular, since $\left(a_{\circ}, a_{\bullet}\right)=\left(p_{k}+1, p_{k}\right)$, it follows from the conclusion of the discussion of $(\mathrm{v})$ that the "link" does not satisfy the condition $\left(\dagger_{2}\right)$ in $(v)$, i.e., that 
the action of $G_{\circ}^{\Gamma}$ on $\operatorname{Aut}\left(\bar{k}_{\bullet}^{\times}\right)$by conjugation-relative to the isomorphism $\operatorname{Aut}\left(\bar{k}_{\circ}^{\times}\right) \stackrel{\sim}{\rightarrow} \operatorname{Aut}\left(\bar{k}_{\bullet}^{\times}\right)$by $\alpha_{\mathrm{Fr}}$-does not preserve the subgroup $\operatorname{Aut}\left(\bar{k}_{\bullet}\right) \subseteq \operatorname{Aut}\left(\bar{k}_{\bullet}^{\times}\right)$.

Therefore, we conclude that

the $\operatorname{subgroup} \operatorname{Aut}\left(\bar{k}_{\bullet}\right) \subseteq \operatorname{Aut}\left(G_{\bullet}\right)$ is not normal,

which thus implies-by considering the respective quotients by $G_{\bullet}$ - that

the $\operatorname{subgroup} \operatorname{Aut}\left(k_{\bullet}\right) \subseteq \operatorname{Out}\left(G_{\bullet}\right)$ is not normal.

This situation is precisely the situation formulated in the "nonnormal portion" of Corollary 8.7.

\section{Acknowledgement}

The author would like to thank the referee for carefully reading the manuscript and giving some helpful comments. This research was supported by the Research Institute for Mathematical Sciences, a Joint Usage/Research Center located in Kyoto University.

\section{References}

[1] Y. Hoshi, A note on the geometricity of open homomorphisms between the absolute Galois groups of $p$-adic local fields, Kodai Math. J. 36 (2013), no. 2, 284-298.

[2] Y. Hoshi, Mono-anabelian reconstruction of number fields, RIMS Preprint 1819 (March 2015).

[3] Y. Hoshi, Introduction to mono-anabelian geometry, RIMS Preprint 1868 (January 2017).

[4] M. Jarden and J. Ritter, On the characterization of local fields by their absolute Galois groups, J. Number Theory 11 (1979), no. 1, 1-13.

[5] M. Jarden and J. Ritter, Normal automorphisms of absolute Galois groups of p-adic fields, Duke Math. J. 47 (1980), no. 1, 47-56.

[6] W. Jenkner, Les corps $p$-adiques dont les groupes de Galois absolus sont isomorphes, Journées Arithmétiques, 1991 (Geneva). Astérisque No. 209 (1992), 14, 221-226.

[7] S. Mochizuki, Topics in absolute anabelian geometry I: generalities, J. Math. Sci. Univ. Tokyo 19 (2012), no. 2, 139-242.

[8] S. Mochizuki, Topics in absolute anabelian geometry III: global reconstruction algorithms, J. Math. Sci. Univ. Tokyo 22 (2015), no. 4, 939-1156.

[9] S. Mochizuki, The mathematics of mutually alien copies: from Gaussian integrals to interuniversal Teichmüller Theory, RIMS Preprint 1854 (July 2016).

[10] N. Nakagoshi, The structure of the multiplicative group of residue classes modulo $\mathfrak{p}^{N+1}$, Nagoya Math. J. 73 (1979), 41-60.

[11] J. Neukirch, Algebraic number theory. Translated from the 1992 German original and with a note by Norbert Schappacher. With a foreword by G. Harder, Grundlehren der Mathematischen Wissenschaften, 322. Springer-Verlag, Berlin, 1999. 
[12] J. Neukirch, A. Schmidt, and K. Wingberg, Cohomology of number fields. Second edition, Grundlehren der Mathematischen Wissenschaften, 323. Springer-Verlag, Berlin, 2008.

[13] J. Ritter, $\mathfrak{B}$-adic fields having the same type of algebraic extensions, Math. Ann. 238 (1978), no. 3, 281-288.

[14] J.-P. Serre, Local fields. Translated from the French by Marvin Jay Greenberg, Graduate Texts in Mathematics, 67. Springer-Verlag, New York-Berlin, 1979.

[15] J.-P. Serre, Abelian $l$-adic representations and elliptic curves. With the collaboration of Willem Kuyk and John Labute, Revised reprint of the 1968 original. Research Notes in Mathematics, 7. A K Peters, Ltd., Wellesley, MA, 1998.

[16] K. Uchida, Isomorphisms of Galois groups, J. Math. Soc. Japan 28 (1976), no. 4, 617-620.

[17] S. Yamagata, A counterexample for the local analogy of a theorem by Iwasawa and Uchida, Proc. Japan Acad. 52 (1976), no. 6, 276-278.

\section{Yuichiro Hoshi}

Research Institute for Mathematical Sciences

Kyoto University

Kyoto 606-8502 Japan

E-mail: yuichiro@kurims.kyoto-u.ac.jp 